LBL-37206

\title{
IMPULSE-BASED METHODS FOR FLUID FLOW ${ }^{1}$
}

\author{
Ricardo Cortez \\ Department of Mathematics \\ and \\ Lawrence Berkeley Laboratory \\ University of California \\ Berkeley, CA 94720, USA
}

Ph.D. Thesis

May 1995

\footnotetext{
1 This dissertation was supported in part by the Applied Mathematical Sciences Subprogram of the Office of Energy Research, U.S. Department of Energy under Contract DE-AC03-76SF00098; by the Air Force Office of Scientific Research AASERT Grant FDF49620-93-1-0053; and by the University of California Affirmative Action Dissertation-year Fellowship.
} 


\section{DISCLAIMER}

This report was prepared as an account of work sponsored by an agency of the United States Government. Neither the United States Government nor any agency thereof, nor any of their employees, make any warranty, express or implied, or assumes any legal liability or responsibility for the accuracy, completeness, or usefulness of any information, apparatus, product, or process disclosed, or represents that its use would not infringe privately owned rights. Reference herein to any specific commercial product, process, or service by trade name, trademark, manufacturer, or otherwise does not necessarily constitute or imply its endorsement, recommendation, or favoring by the United States Government or any agency thereof. The views and opinions of authors expressed herein do not necessarily state or reflect those of the United States Government or any agency thereof. 


\section{DISCLAIMER}

Portions of this document may be illegible in electronic image products. Images are produced from the best available original document. 


\title{
Impulse-Based Particle Methods for Fluid Flow
}

\author{
Copyright $\odot 1995$
}

by

Ricardo Cortez

The U.S. Department of Energy has the right to use this document for any purpose whatsoever including the right to reproduce all or any part thereof 


\author{
Abstract \\ Impulse-based Particle Methods for Fluid Flow \\ by \\ Ricardo Cortez \\ Doctor of Philosophy in Applied Mathematics \\ University of California at Berkeley \\ Professor Alexandre J. Chorin, Chair
}

\begin{abstract}
A Lagrangian numerical method based on impulse variables is analyzed. A relation between impulse vectors and vortex dipoles with a prescribed dipole moment is presented. This relation is used to adapt the high-accuracy cutoff functions of vortex methods for use in impulse-based methods. A source of error in the long-time implementation of the impulse method is explained and two techniques for avoiding this error are presented. An application of impulse methods to the motion of a fluid surrounded by an elastic membrane is presented.
\end{abstract}




\section{Acknowledgements}

I am grateful to have had Alexandre Chorin as my thesis advisor. His encouragement and guidance allowed me to keep focused and confident during the last five years. However, I am most grateful for his invaluable help at every stage of the program and at times when I needed any kind of support. Everyone should have an advisor and friend like him.

I would like to thank my other thesis committee members Jamie Sethian and Phil Colella for having periodic discussions with me and asking questions that pointed me in the right directions. I thank Phil also for always including me in his M. E. group meetings.

There are many other people who made my years in Berkeley very valuable and to whom I owe gratitude. To Ole Hald and John Strain who showed confidence in me, I thank them for their influence and the helpful discussions. To my close friends, my parents, brother, sister and Julia, I'm grateful for their unlimited support over the years. I thank Mike Minion for doing a great job proofreading this thesis. 


\section{Contents}

List of Figures $\quad \mathbf{v}$

List of Tables $\quad$ viii

1 Introduction 1

2 Background and Previous Work $\quad 3$

2.1 Navier-Stokes Equations . . . . . . . . . . . . . . . . . . . . . . . . . . . . . . .

2.1 .1 Velocity Variables . . . . . . . . . . . . . . 3

2.1 .2 Vorticity Variables ................... 4

2.2 Definition of Impulse Variables . . . . . . . . . . . . . . . . . . . 4

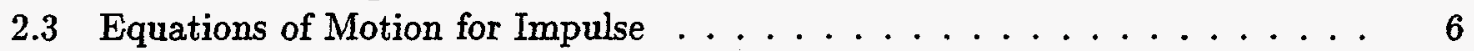

2.3.1 Lagrangian Derivation . . . . . . . . . . . . . 6

2.3.2 Hamiltonian Derivation ................. 8

2.3.3 The Hamiltonian Structure of Fluid Flow . . . . . . . . . . . 9

3 Euler Flow in Impulse Variables 11

3.1 Preliminary Facts About the Flow Map . . . . . . . . . . . . . . . . . 12

3.2 Consequences for Vorticity and Impulse . . . . . . . . . . . . . 13

3.2 .1 Example . . . . . . . . . . . . . . . . . 14

3.3 Orthogonal Surfaces . . . . . . . . . . . . . . . 17

3.3.1 An Example in Two Dimensions . . . . . . . . . . . . . . 18

3.3.2 An Example in Three Dimensions . . . . . . . . . . . . 20

3.3.3 An Example in Three Dimensions . . . . . . . . . . . . . 24

4 A Lagrangian Numerical Method $\quad 25$

4.1 Buttke's Method ... . . . . . . . . . . . . . . . 25

4.1.1 Step 1: The Particle Velocities ... . . . . . . . . . . 26

4.1.2 Step 2: The Rate of Change of Impulse . . . . . . . . . . . 27

4.2 The Hamiltonian Structure of Impulse Flow . . . . . . . . . . . . . . 27

4.3 Comments About the Numerical Method . . . . . . . . . . . 28

4.4 Discrete Impulse and Vortex Blobs . . . . . . . . . . . . . . . 29

4.5 Two-dimensional Impulse and Vortex Dipoles . . . . . . . . . . . 30

4.6 The Velocity Field . . . . . . . . . . . . . . . . . . . 31 


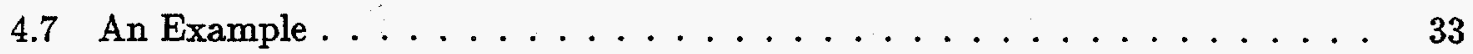

5 Accuracy Considerations in Two Dimensions 37

5.1 Numerical Experiments . . . . . . . . . . . . . . . . . 37

5.1.1 First Experiment: Two Vortex Blobs . . . . . . . . . . . . 38

5.1.2 Second Experiment: Two Vortex Patches . . . . . . . . . . . 40

5.2 Impulse Method With Refinement . . . . . . . . . . . . . . 43

5.2.1 Return to the Example in $\$ 3.7 \ldots \ldots \ldots \ldots . \ldots . \ldots . \ldots 45$

5.3 Impulse Method With Resurfacing . . . . . . . . . . . . . . . . 48

5.3.1 Finding Vortex Positions From the Impulse Discretization . . . . . . 52

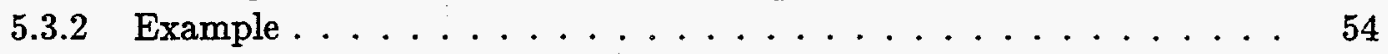

6 Impulse Variables in Three Dimensions $\quad 58$

6.1 Impulse and Vortex Loops . . . . . . . . . . . . . . . . . 58

6.2 Comments . . . . . . . . . . . . . . . . . 60

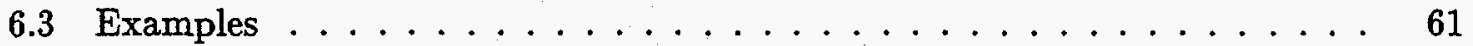

6.3 .1 Hill's Spherical Ring . . . . . . . . . . . . . . 62

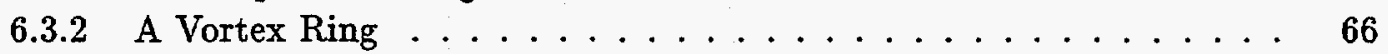

6.4 Impulse Method With Refinement ... . . . . . . . . . . . 67

6.5 Impulse Method With Resurfacing . . . . . . . . . . . . . 70

7 Application of Impulse Methods to Immersed Boundary Problems $\quad 73$

7.1 The Formulation of the Problem . . . . . . . . . . . . . 74

7.2 Boundary Forces . . . . . . . . . . . . . . . . 75

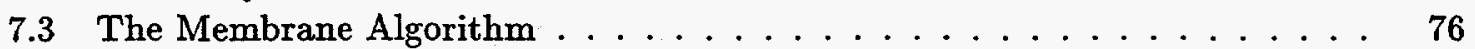

7.4 Numerical Results for Inviscid Flow . . . . . . . . . . . . . . . 77

7.5 The Diffusion Term . . . . . . . . . . . . . . . . . 83

7.6 Numerical Results for Viscous Flow . . . . . . . . . . . . . 84

8 Conclusions $\quad 90$

$\begin{array}{ll}\text { Bibliography } & 93\end{array}$ 


\section{List of Figures}

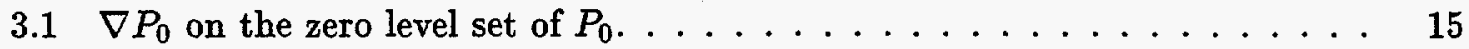

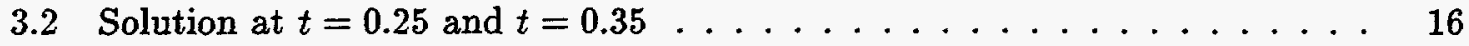

3.3 Initial impulse field. . . . . . . . . . . . . . . . . 19

3.4 Plot of $\nabla P_{t}$ (left) and $\mathbf{m}$ (right) at time $t=6 \ldots \ldots \ldots$

3.5 Plot of $\left|\nabla P_{t}\right|$ (top line) and $|\mathbf{m}|$ (bottom line) as functions of $\theta \ldots \ldots 20$

3.6 Plots of the ratio $\left|\nabla P_{t}\right| /|\mathbf{m}|$ and $(\sin (\theta)+1) / 2 \ldots \ldots \ldots \ldots \ldots$

3.7 Initial impulse (radially symmetric). . . . . . . . . . . . . . . 21

3.8 Impulse field at time $t=2$ (radially symmetric) . . . . . . . . . . 22

3.9 Plot of $\left|\nabla P_{t}\right|$ (dashed) and $|\mathbf{m}|$ (solid) as functions of $s . \ldots \ldots 22$

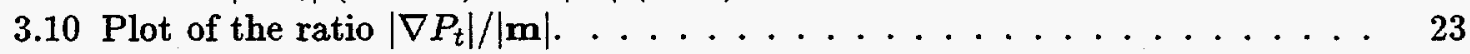

4.1 Vortex Pair Approximation to Impulse Vector. . . . . . . . . . . . . . . 31

4.2 Strip in which vorticity is concentrated. The strip is assumed to have thick-

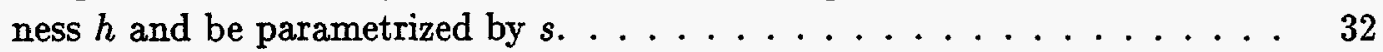

4.3 Two patches of constant vorticity (top). The bottom picture shows the corresponding impulse field defined on a horizontal strip. . . . . . . . . . 34

4.4 Initial m-orthogonal surfaces and the impulse defined on one of them. . . 34

4.5 m-orthogonal surfaces and the impulse defined on one of them at time $t=1$. 35

4.6 m-orthogonal surfaces and the impulse defined on one of them at time $t=3$. 35

5.1 Initial impulse which represents the flow induced by two vortex blobs of equal and opposite strength. . . . . . . . . . . . . . . .

5.2 Solutions at time $t=6$ obtained with a vortex method $(\oplus)$ and the impulse method (arrows) . . . . . . . . . . . . . . . .

5.3 Error in particle positions for the impulse method for time $0 \leq t<10$ and discretizations corresponding to $N=40$ and $N=80$. The error was defined as the average difference in particles positions evolved once with the vortex method and once with the impulse method. . . . . . . . . . . . .

5.4 Solutions at time $t=9$ obtained with a vortex method $(\oplus)$ and the impulse method (arrows) and $N=40 \ldots \ldots \ldots \ldots$

5.5 Initial discretization of the vortex patches with vortices (circles) and location of impulse vectors $($ dots $) . \ldots \ldots \ldots \ldots$ 
5.6 Solution at $t=4.76$. The top figure shows the vortex locations (circles) and the location of the impulse vectors (dots) obtained with the impulse method. The bottom figure shows the location of the vortices obtained with the vortex method. . . . . . . . . . . . . . . . . .

5.7 Refinement procedure which preserves impulse. The vector $\mathbf{m}_{2}$ is replaced by two vectors in the same direction and of half the magnitude of the original. 43

5.8 Solution at $t=4.72$ found with the impulse method with refinement. . . . . 44

5.9 Normalized impulse (dashed) and energy (solid) at $t=4.72$ found with the impulse method with refinement. . . . . . . . . . . . .

5.10 Comparison of vortex positions at $t=4.72$. The top figure shows the distance between corresponding vortices given by the impulse method with refinement for $h=0.05$ (circles) and $h=0.025$ (crosses), compared with the vortex method. The bottom figure shows the ratio of the distances. . . . . . . . .

$5.11 \mathrm{~m}$-orthogonal surfaces and the impulse defined on one of them at time $t=5$. Solution obtained with the impulse method. . . . . . . . . . . .

$5.12 \mathrm{~m}$-orthogonal surfaces and the impulse defined on one of them at time $t=5$. Solution obtained with the impulse method with refinement. . . . . . . .

5.13 Variation in the number of particles used in the impulse method with refinement for the problem of $\$ 3.7 . \ldots \ldots \ldots \ldots$

5.14 Solution at $t=4.72$ found with the impulse method with resurfacing with

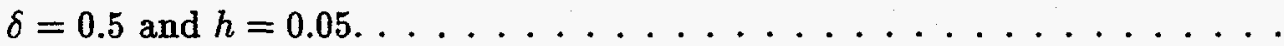

5.15 Variation in the number of particles used in the impulse method with resurfacing for $h=0.025, \delta=0.5$ and $\Delta t=0.08 \ldots \ldots \ldots \ldots$

5.16 The vectors $\mathbf{m}_{1}$ and $\mathbf{m}_{2}$ can be replaced by two pairs of vortices located near the base of the vectors. The left picture shows a low accuracy representation of the impulse flow but allows the combination of the middle vortices. The right picture shows a more accurate representation of the impulse flow by the vortices. . . . . . . . . . . . . . . . .

5.17 Location of impulse vectors before finding vortices. One m-orthogonal curve

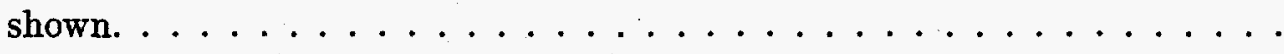

5.18 Location of vortices with $2 h_{0}=0.065$. All impulse vectors allowed the cancellation of vorticity except in the two circular patches. . . . . . . . .

5.19 Location of vortices with $2 h_{0}=0.06$. A few vortices at the top combined but their vorticity did not cancel leaving vortices of strength smaller than 0.0045 .

5.20 Location of vortices with $2 h_{0}=0.055$. Some impulse vectors are not close enough to their neighbors for vortices to combine. The result is pairs of vortices of strength up to \pm 10 inducing nearly opposing flows. . . . . . .

6.1 The impulse vector $\mathbf{m}$ induces the same flow as the vortex loop if $|\mathbf{m}|=A \Gamma$, where $A$ is the area enclosed by the loop and $\Gamma$ is the circulation around the loop. . . . . . . . . . . . . . . . . . .

6.2 A large vortex loop represented as a collection of small loops. . . . . . . .

6.3 Latitudinal curves on the initial sphere. The initial impulse is defined on flat surfaces like the one shown in the top picture. The bottom picture shows the impulse on one $\mathbf{m}$-orthogonal surface at time $t=0 . \ldots \ldots \ldots$ 
6.4 Latitudinal lines on the sphere at $t=1.2$. The $\mathbf{m}$-orthogonal surfaces swell up. The bottom picture shows the impulse on one $\mathbf{m}$-orthogonal surface at

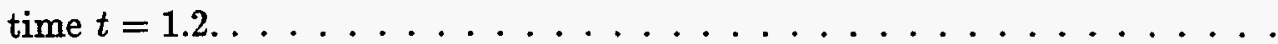

6.5 Front view of the latitudinal lines on the surface of the sphere at time $t=0$ (top) and time $t=1.2$ (bottom). The lines travel away from the north pole.

6.6 Initial vortex ring of radius $R=0.5$ and core radius $a=0.2 \ldots \ldots \ldots$

6.7 Vortex ring of radius $R=0.5$ and core radius $a=0.2$ at time $t=4$. . .

6.8 Figures showing half of an $\mathbf{m}$-orthogonal surface located initially on the $x y$ plane. The surface is shown at times $t=0$ (bottom), $t=2$ (middle) and

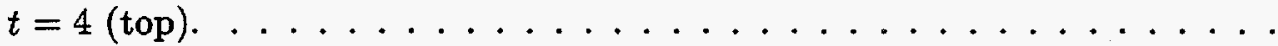

6.9 Refinement of impulse in three dimensions. The impulse vector on the left induces the flow of a vortex loop of area $A$. The refinement process replaces the vector by four vectors of magnitude $1 / 4$ the original one. . . . . . .

6.10 Vortex loops representing an m-orthogonal surface for a ring of $R=1$ and $a=0.4$ at time $t=4$. The top picture shows the loops without refinement; the bottom picture shows the refinement of some loops while others have not yet been refined. . . . . . . . . . . . . . . .

6.11 m-orthogonal surfaces for a vortex ring at time $t=1.0$. The top figure shows one of the original surfaces and the bottom figure shows a new surface after the resurfacing procedure. . . . . . . . . . . . . .

7.1 Initial setup of an elastic membrane surrounding a fluid. . . . . . . . . . 74

7.2 Initial membrane configuration. . . . . . . . . . . . 78

7.3 Motion of the membrane for time $t=0-2.4$, with $\Delta t=0.004 \ldots \ldots 79$

7.4 Motion of the membrane for time $\mathrm{t}=2.8-5.8$, with $\Delta t=0.004 \ldots \ldots . \quad 80$

7.5 Approximate conservation of invariants for the inviscid problem. . . . . . 81

7.6 Impulse field at times $t=1.2$ (top) and $t=5.0$ (bottom) . . . . . . 82

7.7 Initial membrane configuration and particle locations. . . . . . . . . . 84

7.8 Motion of the membrane for time $t=0-2.4$, with $\Delta t=0.004 \ldots \ldots . . \quad 85$

7.9 Motion of the membrane for time $t=2.8-5.52$, with $\Delta t=0.004 . \ldots 86$

7.10 Approximate conservation of invariants for the viscous problem. . . . . . 87

7.11 Impulse field at time $t=2$. The top picture shows the impulse on the membrane; the bottom picture shows the impulse off the membrane (magnified

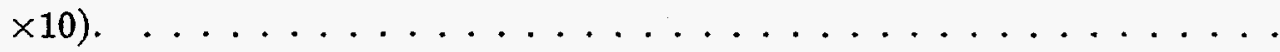

7.12 Impulse field at time $t=5$. The top picture shows the impulse on the membrane; the bottom picture shows the impulse off the membrane (magnified $\times 10)$. 


\section{List of Tables}

5.1 Errors for the vortex patch problem using the impulse method with resurfacing and two different discretizations. The method used cutoff parameter $\delta=0.5$ and time step $\Delta t=0.08 . \mathbf{x}_{V}^{j}$ is the position of the $j$-th particle obtained with the vortex method, $\mathbf{x}_{I}^{j}$ is the position of the same particle obtained with the impulse method. . . . . . . . . . . .

5.2 Errors for the impulse method with resurfacing using three frequencies of resurfacing. The method used cutoff parameter $\delta=0.5, h=0.05$ and time step $\Delta t=0.08 \ldots \ldots \ldots \ldots \ldots \ldots$

5.3 Errors for the vortex patch problem using the impulse method with resurfacing and two different time steps. The method used $\delta=0.25$ and discretization

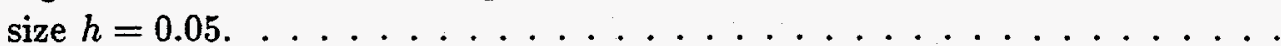




\section{Chapter 1}

\section{Introduction}

The Navier-Stokes equations, which describe the motion of viscous fluids, are typically written in terms of the fluid velocity or vorticity. Most numerical methods are discretizations of either formulation of these equations. In recent years, the introduction of a new variable, which has been called magnetization [6, 8], velicity [7], impetus [24], and impulse [17], led to a new formulation of the fluid equations [29, 7]. In terms of the new variable, the Euler equations, which describe the motion of inviscid fluids, display readily the Hamiltonian structure of fluid flow in any space dimension. Buttke [7] presented a Lagrangian numerical method based on discretizations of these new equations. These discretizations lead to systems which in the absence of viscosity, conserve many invariants associated with the Hamiltonian, including kinetic energy, impulse, helicity, and angular momentum.

The numerical method based on impulse, presented by Buttke, is the starting point for this dissertation. Many questions regarding the method have been unresolved. For instance, specific flows for which the method is particularly useful have not been identified; the accuracy of impulse-based methods has not been studied for long-time simulations; convergence properties of the method were unknown; how to choose the cutoff functions was not know. These questions are answered here.

The dissertation begins with background material about the Navier-Stokes equations, the definition of impulse, its equation of motion, and the relevant Hamiltonian formalism. In chapter 3 , the equations of motion for impulse are used to obtain some results about the evolution of impulse, and geometric interpretations of impulse flow are made with the help of surfaces orthogonal to the impulse field. The numerical method and its properties 
are presented in chapter 4 , as is the connection between impulse and vortex dipoles. This connection validates the use of the cutoff functions of vortex methods in impulse methods and sheds light on the accuracy problems that can be expected in some long-time implementations of the method. The special case of two-dimensional flow is discussed in chapter 5 , where two techniques for maintaining long-time accuracy are presented and illustrated by numerical examples. Chapter 6 contains a discussion of three-dimensional implementation of the method. Finally, in chapter 7, an important application of impulse methods to the motion of a two-dimensional elastic membrane surrounding an incompressible fluid is presented for the cases of both inviscid and viscous fluids. This is an example of an application for which impulse methods are particularly well-suited and is a step toward the use of impulse methods in physiological flows. 


\section{Chapter 2}

\section{Background and Previous Work}

\subsection{Navier-Stokes Equations}

The equations that describe the motion of viscous fluids are the Navier-Stokes equations. In this chapter, the Navier-Stokes equations for incompressible fluids are presented in three different forms. They are introduced in terms of the fluid velocity, which is the form that naturally arises from equating the rate of change of momentum of a portion of fluid to the sum of all forces acting on the same portion of fluid. The vorticity in the flow is then defined and the evolution equations for the vorticity are deduced. Finally, impulse variables are introduced and the Navier-Stokes equations expressed in terms of impulse are derived.

\subsubsection{Velocity Variables}

Let $\Omega$ be a subset of $R^{2}$ or $R^{3}$ with smooth solid boundary $\partial \Omega$. If the fluid velocity is denoted by $\mathbf{u}=\left(u_{1}, u_{2}, u_{3}\right)$, the Navier-Stokes equations for incompressible fluids are

$$
\begin{aligned}
\mathbf{u}_{t}+(\mathbf{u} \cdot \nabla) \mathbf{u} & =-\nabla p+\nu \Delta \mathbf{u} \\
\nabla \cdot \mathbf{u} & =\mathbf{0}
\end{aligned}
$$

in $\Omega$. Here, $t$ is time, $\mathbf{u}_{t}$ is shorthand notation for $\partial \mathbf{u} / \partial t, p$ is the pressure, and $\nu$ is the fluid viscosity. Eq. (2.2) expresses the incompressibility of the fluid. The viscosity will force the fluid to come to a stop at the boundary of the domain, so the equations must be supplemented by the boundary condition $\mathbf{u}=0$ on $\partial \Omega$. For inviscid fluids (i.e. $\nu=0$ ), the 
lack of friction allows the fluid to move along the solid boundary but not to penetrate it. In this case, the boundary condition is $\mathbf{u} \cdot \hat{\mathbf{n}}=0$ on $\partial \Omega$, where $\hat{\mathbf{n}}$ is the outward unit vector normal to the boundary. When the domain $\Omega$ is the entire space, the boundary condition is replaced by $\mathbf{u} \rightarrow 0$ as $|\mathbf{x}| \rightarrow \infty$. The free-space case will be considered from now on.

\subsubsection{Vorticity Variables}

The vorticity $\xi$ of a flow is defined as the curl of the fluid velocity, $\xi=\nabla \times \mathbf{u}$. If one thinks of $\mathrm{R}^{2}$ as a subspace of $\mathrm{R}^{3}$, then in two dimensions the vorticity is a vector normal to the plane; one could then write $\xi=(0,0, \omega)^{T}$, where $\omega=\partial_{x_{1}} u_{2}-\partial_{x_{2}} u_{1}$, and $\left(x_{1}, x_{2}, x_{3}\right)$ are the coordinate variables.

By taking the curl of Eq. (2.1) and using Eq. (2.2), one arrives at the equations of motion for incompressible flow in terms of vorticity [16]

$$
\xi_{t}+(\mathbf{u} \cdot \nabla) \xi=(\xi \cdot \nabla) \mathbf{u}+\nu \Delta \xi
$$

In two dimensions, the first term of the right-hand side is zero, so the equations reduce to the scalar equation

$$
\omega_{t}+(\mathbf{u} \cdot \nabla) \omega=\nu \Delta \omega
$$

Eq. (2.4) indicates that, in the absence of viscosity (i.e. for $\nu=0$ ), the vorticity is simply transported by the flow; that is, $\omega$ remains constant along particle trajectories.

In three dimensions, the vorticity along particle trajectories can change even in the absence of viscosity due to the first term on the right-hand side of Eq. (2.3). This term is commonly called the stretching term, because it is associated with the stretching of vortex lines [16].

\subsection{Definition of Impulse Variables}

A key element in the discussion of impulse and its equations of motion is the following decomposition theorem (see e.g. [16]):

Theorem 1 (Hodge Decomposition) Let $\Omega$ be a simply connected, bounded domain with smooth boundary $\partial \Omega$. Then, any vector-valued function $\mathbf{q}$ defined on $\Omega$ can be written uniquely as the sum

$$
\mathbf{q}=\mathbf{u}+\nabla \zeta
$$


with $\nabla \cdot \mathbf{u}=0$ in $\Omega$ and $\mathbf{u} \cdot \hat{\mathbf{n}}=0$ on $\partial \Omega$, where $\hat{\mathbf{n}}$ is the outward unit normal to $\partial \Omega$.

The two terms in the decomposition of $\mathbf{q}$ are orthogonal in the sense that $\int_{\Omega} \mathbf{u} \cdot \nabla \zeta d V=0$, where $d V$ is an element of volume. The divergence-free part, $\mathbf{u}$, is called the orthogonal projection of $\mathrm{q}$ on the space of divergence-free vectors tangent to $\partial \Omega$, and is represented by $\mathbf{u}=\mathbb{P q}$. In free space, the boundary condition $\mathbf{u} \cdot \hat{\mathbf{n}}=\mathbf{0}$ is replaced by the decay condition $\mathbf{u} \rightarrow 0$ as $|\mathbf{x}| \rightarrow \infty$.

One way to introduce impulse is to start with the fluid velocity at time $t=0$ and make a change of variables in the following way: let $\mathbf{m}$ be a vector field equivalent to $\mathbf{u} \mathbf{u p}$ to an arbitrary gradient; that is,

$$
\mathbf{m}=\mathbf{u}+\nabla \phi
$$

\section{Remarks}

1. $m$ has units of velocity but it is not the velocity of the fluid.

2. Eq. (2.5) implies that $\mathbf{u}$ is the divergence-free part of the Hodge decomposition of $\mathbf{m}$ in free space.

3. $\mathbf{m}$ is not uniquely defined since the function $\phi$ is initially arbitrary. Consequently, $\mathbf{m}$ can be transformed according to $\mathbf{m} \rightarrow \mathbf{m}+\nabla \bar{\phi}$, for any arbitrary scalar function $\tilde{\phi}$. This transformation is called a gauge transformation.

4. The vorticity $\xi$ can be found from either $\mathbf{u}$ or $\mathbf{m}$ since $\xi=\nabla \times \mathbf{u}=\nabla \times \mathbf{m}$.

Impulse, $\mathbf{I}$, is the total amount of linear momentum required to start the given motion from rest (see [2]). Impulse is given by

$$
\mathrm{I}=\frac{\rho}{2} \int_{\mathrm{R}^{3}} \mathrm{x} \times \xi d V
$$

The fluid density will be assumed to be $\rho \equiv 1$ from now on. If $\mathbf{m}$ has compact support, one obtains from the above definition and Eq. (2.5)

$$
\mathbf{I}=\frac{1}{2} \int_{\mathrm{R}^{3}} \mathbf{x} \times \xi d V=\frac{1}{2} \int_{\mathrm{R}^{3}} \mathbf{x} \times(\nabla \times \mathbf{m}) d V=\int_{\mathrm{R}^{3}} \mathbf{m} d V,
$$

where the last equality follows after integration by parts. It follows that $\mathbf{m}$ is an impulse density, thus $\mathbf{m}$ will be referred to as impulse. The literature refers to this same variable m also as magnetization and velicity $[7,8,14]$. 
Consider now a two-dimensional impulse field $\mathbf{m}(\mathbf{x})$ with support inside a bounded set $\Omega$. It follows that

$$
\int_{\partial \Omega} \mathbf{m} \cdot d \mathbf{l}=0
$$

where $d l$ is a length element tangent to the boundary. By Stokes' theorem,

$$
0=\int_{\partial \Omega} \mathbf{m} \cdot d \mathbf{l}=\int_{\Omega}(\nabla \times \mathbf{m}) \cdot d \mathbf{A}=\int_{\Omega} \xi \cdot d \mathbf{A}
$$

Therefore, only when the net vorticity is zero can $\mathbf{m}(\mathbf{x})$ have compact support in two dimensions. Consequently, a patch of positive vorticity cannot be represented by a compactly supported impulse field.

In order to make use of the change of variables defined in Eq. (2.5), one would like to derive evolution equations for $m$ with the property that the velocity field deduced from $\mathbf{m}$ by the Hodge decomposition satisfies the Navier-Stokes equations (2.1)-(2.2).

\subsection{Equations of Motion for Impulse}

The fluid equations of motion in impulse variables can be derived in various ways. One way is to start with the definition of impulse, Eq. (2.5), and use the Navier-Stokes equations in velocity variables. A second way is to use a Hamiltonian formulation for incompressible fluid flow [24]. Both methods are presented here for flows in free space. A third method for deriving the equations of motion for impulse uses Eq. (2.6) as the starting point; see [6] for more details.

\subsubsection{Lagrangian Derivation}

If one considers any function of position and time, it is convenient to define the rate of change of this function along the particles paths.

Definition. The material derivative of any quantity $g$ is $D g / D t=\partial g / \partial t+\mathbf{u} \cdot \nabla g$.

The material derivative represents the rate of change of a function at a point moving with the fluid. Using the material derivative, Eq. (2.1) can be written as

$$
\frac{D \mathbf{u}}{D t}=-\nabla p+\nu \Delta \mathbf{u}
$$


Since $\mathbb{P} \nabla \mathbf{f}=0$ for any continuously differentiable function $f$, applying the projection operator to the last equation yields

$$
\mathbb{P} \frac{\mathrm{Du}}{\mathrm{D} t}=\mathbb{P} \nu \Delta \mathbf{u}=\mathbb{P} \nu \Delta \mathbf{m}
$$

The following identities are useful in the derivations of the equations in this chapter:

(ID1) $\nabla\left(\frac{1}{2}|\mathbf{u}|^{2}\right)=(\nabla \mathbf{u})^{T} \mathbf{u}$

(ID2) $\frac{D}{D t} \nabla \phi=\nabla\left(\frac{D \phi}{D t}\right)-(\nabla \mathbf{u})^{T} \nabla \phi$,

where $\nabla \mathbf{u}$ is a matrix with entries $(\nabla \mathbf{u})_{i j}=\partial u_{i} / \partial x_{j}$, and $T$ denotes a transpose. From Eq. (2.5), one finds that impulse satisfies $[7,8]$

$$
\begin{aligned}
\frac{D \mathbf{m}}{D t} & =\frac{D \mathbf{u}}{D t}+\frac{D}{D t} \nabla \phi=\frac{D \mathbf{u}}{D t}+\nabla\left(\frac{D \phi}{D t}\right)-(\nabla \mathbf{u})^{T} \nabla \phi \\
& =\frac{D \mathbf{u}}{D t}+\nabla\left(\frac{D \phi}{D t}+\frac{1}{2}|\mathbf{u}|^{2}\right)-(\nabla \mathbf{u})^{T} \mathbf{m}
\end{aligned}
$$

where (ID1) and (ID2) have been used. One can write Eq. (2.7) in the form

$$
\begin{aligned}
\frac{D \mathbf{u}}{D t} & =-\nabla p+\nu \Delta[\mathbf{m}-\nabla \phi] \\
& =-\nabla[p+\nu \Delta \phi]+\nu \Delta \mathbf{m}
\end{aligned}
$$

and substitute this expression in Eq. (2.9) to obtain

$$
\frac{D \mathbf{m}}{D t}=\nabla\left(\frac{D \phi}{D t}+\frac{1}{2}|\mathbf{u}|^{2}-p-\nu \Delta \phi\right)-(\nabla \mathbf{u})^{T} \mathbf{m}+\nu \Delta \mathbf{m} .
$$

This is an equation of motion for $\mathbf{m}$ given any initial choice of the function $\phi$. If one lets $\phi$ evolve according to

$$
\frac{D \phi}{D t}+\frac{1}{2}|\mathbf{u}|^{2}-p-\nu \Delta \phi=0
$$

the equation of motion for $\mathbf{m}$ in free space reduces to

$$
\begin{aligned}
& \frac{D \mathbf{m}}{D t}=-(\nabla \mathbf{u})^{T} \mathbf{m}+\nu \Delta \mathbf{m} \\
& \mathbb{P m}=\mathbf{u} .
\end{aligned}
$$

The specific choice of the equation of motion for $\phi$ above leads to Eq. (2.10). This particular form of the equation for $\mathbf{m}$ arises naturally in the Hamiltonian derivation of the next section and is the form that allows the geometric interpretations of impulse flows discussed in the 
next chapter. It is easy to show that Eq. (2.8) can be obtained by applying the projection operator to Eq. (2.10). This is done at the end of the next section.

It is important to make the connection between $\mathbf{m}$ and computational variables with similar properties used in other numerical methods. For instance, projection methods $[11,12]$ generally update the fluid velocity in two steps: a first velocity update is obtained by approximating Eq. (2.7) without regard to the divergence constraint; the update is then projected onto the space of divergence-free vector fields to find the velocity. The auxiliary field obtained after the first step is an impulse field since its divergence-free part is the fluid velocity. For more details on projection methods see $[11,12,4]$.

\subsubsection{Hamiltonian Derivation}

The Hamiltonian structure of Euler flow in impulse variables will be discussed in its discrete version when the numerical method is presented. In this section, a simplified version of the derivation of the Hamiltonian equations found in [24] is presented.

Let $\mathbf{x}(\alpha, t)$ denote the coordinates at time $t$ of a material particle located initially at position $\alpha$, and let $\dot{\mathbf{x}}=\mathbf{u}(\mathbf{x}, t)$ be the velocity. For an inviscid fluid, one starts with the Lagrangian $L(\mathbf{x}, \dot{\mathbf{x}}, \alpha)=\int \frac{1}{2} \dot{\mathbf{x}}^{2} J d \alpha$, where $J$ is the Jacobian of the map $\alpha \longmapsto \mathbf{x}$. The constraint $\nabla \cdot \dot{\mathbf{x}}=0$ can be introduced with a Lagrange multiplier [25,24], $\phi(\alpha, t)$, in the full variational Lagrangian

$$
\mathcal{L}(\mathbf{x}, \dot{\mathbf{x}}, \phi)=\int\left[\frac{1}{2} \dot{\mathbf{x}}^{2}-(\nabla \cdot \dot{\mathbf{x}}) \phi(\alpha, t)\right] J d \alpha .
$$

From this, it can be shown that $\delta \mathcal{L} / \delta \dot{\mathbf{x}}=\dot{\mathbf{x}}+\nabla \phi=\mathbf{u}+\nabla \phi$. This means that $\mathbf{m}=\mathbf{u}+\nabla \phi$ is the appropriate variable conjugate to position. The constraint $\delta \mathcal{L} / \delta \phi=0$ yields the incompressibility condition $\nabla \cdot \mathbf{u}=0$.

One can expect to find a Hamiltonian which will lead to equations of motion for $\mathbf{x}$ and $\mathbf{m}$. The canonical Hamiltonian equations [24] are

$$
\begin{aligned}
\dot{\mathbf{x}}(t) & =\mathbf{u}(\mathbf{x}) \\
\dot{\mathbf{m}}(t) & =-\left[(\nabla \mathbf{u})^{T} \nabla \phi+\nabla\left(\frac{1}{2}|\mathbf{u}|^{2}\right)\right] J \\
& =-\left[(\nabla \mathbf{u})^{T} \mathbf{m}\right] J
\end{aligned}
$$

from which one can deduce that $J \equiv 1$ (see [24]). 
In the case of viscous fluids, one expects the equations to be

$$
\begin{aligned}
\dot{\mathbf{x}}(t) & =\mathbf{u}(\mathbf{x}) \\
\dot{\mathbf{m}}(t) & =-(\nabla \mathbf{u})^{T} \mathbf{m}+\nu \Delta \mathbf{m} .
\end{aligned}
$$

Indeed, by replacing $\mathbf{m}=\mathbf{u}+\nabla \phi$ and using (ID1) and (ID2), one obtains

$$
\begin{aligned}
\frac{D \mathbf{u}}{D t} & =\frac{D}{D t}(\mathbf{m}-\nabla \phi)=\frac{D \mathbf{m}}{D t}-\nabla \frac{D \phi}{D t}+(\nabla \mathbf{u})^{T} \nabla \phi \\
& =-\left[(\nabla \mathbf{u})^{T} \mathbf{u}+(\nabla \mathbf{u})^{T} \nabla \phi\right]+\nu[\Delta \mathbf{u}+\Delta(\nabla \phi)]-\nabla \frac{D \phi}{D t}+(\nabla \mathbf{u})^{T} \nabla \phi \\
& =-\nabla\left(\frac{D \phi}{D t}+\frac{1}{2}|\mathbf{u}|^{2}-\nu \Delta \phi\right)+\nu \Delta \mathbf{u},
\end{aligned}
$$

which yields the Navier-Stokes equations in projection form, Eq. (2.8), after multiplication by the projection operator $\mathbb{P}$.

Discretizations of Eq. (2.14)-(2.15) lead to Lagrangian numerical methods in terms of impulse variables. Methods resulting from a particular approximation of the impulse field will be discussed in chapter 4 .

\subsubsection{The Hamiltonian Structure of Fluid Flow}

The Lagrangian $L(\mathbf{x}, \dot{\mathbf{x}}, \alpha)=\int \frac{1}{2} \mathbf{u}^{2} d V$ was the starting point for the Hamiltonian derivation of the evolution equations for impulse in the last section. Based on this Lagrangian, one can define the Hamiltonian in terms of velocity and impulse by

$$
H(\mathbf{u}, \mathbf{m})=\int\left[\mathbf{m} \cdot \mathbf{u}-\frac{1}{2} \mathbf{u} \cdot \mathbf{u}\right] d V
$$

In order to introduce the constraint $\nabla \cdot \mathbf{u}=0$, let $\mathbf{u}=\mathbf{m}-\nabla \phi$ and replace $(\mathbf{u} \cdot \mathbf{u})$ by $[\mathbf{u} \cdot(\mathbf{m}-\nabla \phi)]$ in the second term of the Hamiltonian. This yields

$$
H(\mathbf{u}, \mathbf{m})=\int\left[\mathbf{m} \cdot \mathbf{u}-\frac{1}{2} \mathbf{u} \cdot(\mathbf{m}-\nabla \phi)\right] d V=\frac{1}{2} \int \mathbf{m} \cdot \mathbf{u} d V
$$

where the last equality follows after integration by parts. Note that the Hamiltonian can also be written as $\frac{1}{2} \int \mathbf{u} \cdot \mathbf{u} d V$ and thus represents the kinetic energy of the fluid $[7,8]$.

In the case of an inviscid fluid, many quantities are known to be invariants of the flow. The invariance of kinetic energy and impulse is presented here under the assumption that $\mathbf{m}$ has compact support inside a set $\Omega$. The rate of change of kinetic energy is

$$
\frac{D H}{D t}=\frac{1}{2} \frac{d}{d t} \int \mathbf{u} \cdot \mathbf{u} d V=\int \mathbf{u}_{t} \cdot \mathbf{u} d V=\int_{\Omega} \mathbf{m}_{t} \cdot \mathbf{u} d V
$$


For incompressible flow, one can verify the identity $\left[(\nabla \mathbf{u})^{T} \mathbf{m}+(\mathbf{u} \cdot \nabla) \mathbf{m}\right] \cdot \mathbf{u}=\nabla \cdot[(\mathbf{u} \cdot \mathbf{m}) \mathbf{u}]$. This formula is used to conclude that

$$
\begin{aligned}
\frac{D H}{D t} & =-\int_{\Omega}\left[(\nabla \mathbf{u})^{T} \mathbf{m}+(\mathbf{u} \cdot \nabla) \mathbf{m}\right] \cdot \mathbf{u} d V=-\int_{\Omega} \nabla \cdot[(\mathbf{u} \cdot \mathbf{m}) \mathbf{u}] d V \\
& =-\int_{\partial \Omega}(\mathbf{u} \cdot \mathbf{m})(\mathbf{u} \cdot \hat{\mathbf{n}}) d S=0
\end{aligned}
$$

since $\mathbf{u} \cdot \hat{\mathbf{n}}=0$ on $\partial \Omega$ by the Hodge decomposition.

The invariance of impulse can also be established via Eq. (2.15). Recall that

$$
\mathbf{I}=1 / 2 \int_{\mathbf{R}^{3}} \mathbf{x} \times \xi d V=\int_{\Omega} \mathbf{m} d V
$$

Differentiating with respect to time, one finds

$$
\frac{D \mathbf{I}}{D t}=\int_{\Omega} \mathbf{m}_{t} d V=-\int_{\Omega}(\nabla \mathbf{u})^{T} \mathbf{m}+(\mathbf{u} \cdot \nabla) \mathbf{m} d V
$$

One can verify the following formula for any sufficiently smooth vector field $\mathbf{q}$,

$$
\int_{\Omega}(\mathbf{u} \cdot \nabla) \mathbf{q} d V=\int_{\partial \Omega} \mathbf{q}(\mathbf{u} \cdot \hat{\mathbf{n}}) d S-\int_{\Omega} \mathbf{q}(\nabla \cdot \mathbf{u}) d V=0 .
$$

Therefore, the last term in the equation for $D \mathbf{I} / D t$ is zero and

$$
\begin{aligned}
\frac{D \mathbf{I}}{D t} & =-\int_{\Omega}(\nabla \mathbf{u})^{T} \mathbf{m} d V=\int_{\Omega} \mathbf{u} \times(\nabla \times \mathbf{m}) d V \\
& =\int_{\Omega} \mathbf{u} \times(\nabla \times \mathbf{u}) d V \\
& =\int_{\Omega} \nabla\left(\frac{1}{2}|\mathbf{u}|^{2}\right)-(\mathbf{u} \cdot \nabla) \mathbf{u} d V=\mathbf{0}
\end{aligned}
$$

Incompressible Euler flow has other known invariants which can be expressed in terms of impulse. The integral $\int \xi \cdot \mathbf{u} d V$ is known as the helicity. In terms of impulse, the helicity can be written as $\int_{\Omega} \xi \cdot \mathbf{m} d V$. In the next chapter, it will be shown that the helicity density is constant along particle paths. This implies that $\int F(\xi \cdot \mathbf{m}) d V$, where $F$ is any sufficiently smooth function, is a conserved quantity (see e.g. [29]).

Other invariants of Euler flow are the angular momentum, defined as $\int \mathbf{x} \times \mathbf{u} d V$ and the circulation around any closed curve moving with the flow, $\int_{C} \mathbf{u} \cdot d \mathbf{l}$, where $d \mathbf{l}$ is an element of length along the curve (see $[16,2]$ ). These can be written respectively in terms of impulse as $\int \mathbf{x} \times \mathbf{m} d V$ (see [7]) and $\int_{C} \mathbf{m} \cdot d \mathbf{l}$. 


\section{Chapter 3}

\section{Euler Flow in Impulse Variables}

The topic of this chapter is fluid flow in the case of zero viscosity. In this case, the equations are the Euler equations. The vorticity was defined in the previous chapter as the curl of the fluid velocity, $\xi=\nabla \times \mathbf{u}$. The Euler equations for vorticity along particle trajectories are a special case of Eq. (2.3),

$$
\frac{D \xi}{D t}=(\nabla \mathbf{u}) \xi
$$

The right-hand side describes how the vorticity carried by fluid particles changes in time. In two dimensions, the only non-zero component of vorticity is the one normal to the plane. This scalar, denoted $\omega$, satisfies the Euler equation

$$
\frac{D \omega}{D t}=0
$$

which states that the vorticity carried by fluid particles does not change in time. This is Eq. (2.4) without the viscous term.

Impulse was defined as a vector function whose divergence-free component is the fluid velocity. The Euler equations in two or three dimensions in terms of impulse are

$$
\frac{D \mathbf{m}}{D t}=-(\nabla \mathbf{u})^{T} \mathbf{m}
$$

In either two or three dimensions, impulse carried by the fluid particles may change in time according to the equation above.

In a Lagrangian numerical method one tracks the flow properties along trajectories traced in time by fluid particles. As a prelude to the numerical method, some results regarding vorticity and impulse carried by the particles are presented now. A connection 
between the equations of motion for impulse and the equations of motion for gradients of certain functions will give rise to interesting geometric interpretations.

\subsection{Preliminary Facts About the Flow Map}

This section presents results about vorticity and impulse in the case of Euler flow. The results make use of a map which advances each fluid particle from its position at time $t=0$ to its position at later times $[16]$.

Definition. Let $\phi_{t}$ denote the map which, for a given $t$, maps each fluid particle from its initial position to its position at time $t$. If $\mathbf{x}(\alpha, t)$ is the trajectory followed by the particle which is initially at position $\alpha$, then $\phi_{t}$ sends $\alpha$ to $\mathbf{x}(\alpha, t)$. The Jacobian matrix of the map $\phi_{t}$ is a function of space and time and will be denoted $\nabla \phi_{t}$. (The subscript indicates the dependence in time, not differentiation.)

All functions, including the fluid velocity, vorticity, impulse and flow map, will be assumed to be as smooth as required for the propositions in this chapter. The first proposition is a simple consequence of the definition of the flow map. The second one shows how the vorticity at a point $\mathbf{x}(\alpha, t)$ depends on the flow map and on the initial vorticity at $\alpha$. Both propositions are found in [16].

Proposition $1 \frac{\partial}{\partial t}\left(\nabla \phi_{t}\right)=(\nabla \mathbf{u})\left(\nabla \phi_{t}\right)$

Proof:

$$
\begin{aligned}
\frac{\partial}{\partial t}\left(\nabla \phi_{t}\right)_{i j} & =\left(\nabla\left[\frac{\partial x}{\partial t}(\alpha, t)\right]\right)_{i j}=(\nabla(\mathbf{u}(\dot{x}, t)))_{i j} \\
& =\left(\partial u_{i} / \partial x_{k}\right)\left(\partial x_{k} / \partial \alpha_{j}\right)
\end{aligned}
$$

Thus $\frac{\partial}{\partial t}\left(\nabla \phi_{t}\right)_{i j}=(\nabla \mathbf{u})_{i k}\left(\nabla \phi_{t}\right)_{k j}=\left((\nabla \mathbf{u})\left(\nabla \phi_{t}\right)\right)_{i j}$.

It was already mentioned that in two dimensions, $\xi(\mathbf{x}(\alpha, t), t)=\xi(\alpha, 0)$. The following result establishes the relationship between the vorticity at a point $\mathbf{x}(\alpha, t)$ and time $t$, and the vorticity at the point $\alpha$ at $t=0$ in three dimensions.

Proposition 2 In three dimensions, $\xi(x, t)=\left(\nabla \phi_{t}\right) \xi(\alpha, 0)$. 
Proof:

$$
\begin{aligned}
\frac{D}{D t}\left[\xi-\left(\nabla \phi_{t}\right) \xi(\alpha, 0)\right] & =\frac{D \xi}{D t}-\left[\frac{\partial}{\partial t}\left(\nabla \phi_{t}\right)\right] \xi(\alpha, 0) \\
& =\frac{D \xi}{D t}-\left[(\nabla \mathbf{u})\left(\nabla \phi_{t}\right)\right] \xi(\alpha, 0) \\
& =(\nabla \mathbf{u}) \xi-(\nabla \mathbf{u})\left(\nabla \phi_{t}\right) \xi(\alpha, 0) \\
& =(\nabla \mathbf{u})\left[\xi-\left(\nabla \phi_{t}\right) \xi(\alpha, 0)\right]
\end{aligned}
$$

Since $\left(\nabla \phi_{t}\right)=I$ at $t=0$, the expression in brackets is zero for all time.

A similar result can be established for impulse in either two or three dimensions [7].

Proposition $3 \mathbf{m}(x, t)=\left(\nabla \phi_{t}\right)^{-T} \mathbf{m}(\alpha, 0)$

Proof:

$$
\begin{aligned}
\frac{D}{D t}\left[\left(\nabla \phi_{t}\right)^{T} \mathbf{m}\right] & =\left[\frac{\partial}{\partial t}\left(\nabla \phi_{t}\right)\right]^{T} \mathbf{m}+\left(\nabla \phi_{t}\right)^{T} \frac{D \mathbf{m}}{D t} \\
& =\left(\nabla \phi_{t}\right)^{T}(\nabla \mathbf{u})^{T} \mathbf{m}+\left(\nabla \phi_{t}\right)^{T}(-\nabla \mathbf{u})^{T} \mathbf{m} \\
& =0
\end{aligned}
$$

The result follows since $\left(\nabla \phi_{0}\right)=I$.

It is clear that the given form of the equation for $\mathbf{m}$ plays a key role in this proposition and, as will be seen in the next section, in the geometric interpretations of impulse flow.

\subsection{Consequences for Vorticity and Impulse}

In two dimensions, the vorticity $\xi$ is characterized by the scalar $\omega$, where $\xi=$ $(0,0, \omega)^{T}$. Since vorticity is normal to the $x y$-plane, the helicity density $(\xi \cdot \mathbf{m})$ is identically zero. In three dimensions, both $\xi$ and $\mathbf{m}$ are vector fields and the helicity density is generally a function of time and space. From the previous propositions, one can establish the following result found in [29].

Proposition $4 \xi(x, t) \cdot \mathbf{m}(x, t)=\xi(\alpha, 0) \cdot \mathbf{m}(\alpha, 0)$. 
Proof:

$$
\begin{aligned}
\xi(x, t) \cdot \mathbf{m}(x, t) & =\left[\left(\nabla \phi_{t}\right) \xi(\alpha, 0)\right] \cdot\left[\left(\nabla \phi_{t}\right)^{-T} \mathbf{m}(\alpha, 0)\right] \\
& =\xi(\alpha, 0) \cdot \mathbf{m}(\alpha, 0)
\end{aligned}
$$

This proposition establishes that $(\xi \cdot \mathbf{m})$ is constant along particle paths. Consequently, for any smooth scalar function $F$, the quantity $F(\xi \cdot \mathbf{m})$ is conserved in Euler flow. The next proposition shows that one can define families of functions, parametrized by time, so that the gradients of those functions evolve in time with the same equation as $\mathbf{m}$.

Proposition 5 Let $P_{0}: \mathrm{R}^{n} \rightarrow \mathrm{R}(n=2$ or 3 ) be smooth and define the family of functions $P_{t}(\mathbf{x})=P_{0} \circ \phi_{t}^{-1}(\mathbf{x})$, parametrized by time. Then the evolution equation for $\nabla P_{t}$ is identical to the evolution equation for $\mathbf{m}$. Furthermore, $\nabla P_{t}(\mathbf{x})=\left(\nabla \phi_{t}\right)^{-T} \nabla P_{0}(\alpha)$ for all $t \geq 0$.

Proof: One can write $P_{t} \circ \phi_{t}(\alpha)=P_{0}(\alpha)$. Differentiation of this equation with respect to $\alpha$ yields $\left(\nabla \phi_{t}\right)^{T} \nabla P_{t}(\mathbf{x})=\left(\nabla \phi_{0}\right)^{T} \nabla P_{0}(\alpha)$, where the fact $\left(\nabla \phi_{0}\right)=I$ has been used. The expression $\left(\nabla \phi_{t}\right)^{T} \nabla P_{t}(\mathbf{x})$ is invariant in time, thus

$$
\begin{aligned}
0=\frac{D}{D t}\left[\left(\nabla \phi_{t}\right)^{T} \nabla P_{t}(\mathbf{x})\right] & =\left(\nabla \phi_{t}\right)^{T}(\nabla \mathbf{u})^{T} \nabla P_{t}(\mathbf{x})+\left(\nabla \phi_{t}\right)^{T} \frac{D}{D t} \nabla P_{t}(\mathbf{x}) \\
\Rightarrow \frac{D}{D t} \nabla P_{t}(\mathbf{x}) & =-(\nabla \mathbf{u})^{T} \nabla P_{t}(\mathbf{x})
\end{aligned}
$$

This result indicates that the gradient of the time-dependent function $P_{t}$ will evolve with the same equation of motion as $\mathbf{m}$. Then one can deduce that if $\mathbf{m}(\alpha, 0)=\nabla P_{0}(\alpha)$ for some $P_{0}$, then $\mathbf{m}(\mathbf{x}, t)$ will equal $\nabla P_{t}(\mathbf{x})$ for all $t \geq 0$. Therefore, if $\mathbf{m}$ is initially a gradient (i.e. the flow is irrotational), $\mathbf{m}$ will remain a gradient. This is a statement that in free space, no vorticity is created. Under the same assumptions, since $\nabla P_{t}$ is perpendicular to the zero level set of $P_{t}$ (for any $t$ ), as this level set deforms when it is carried by the flow, $\nabla P_{t}$ and therefore $\mathbf{m}$, will remain perpendicular to the level set.

\subsubsection{Example}

Consider two-dimensional steady flow given by $\mathbf{u}(x, y)=\left(1-e^{-x}, e^{y}\right)^{T}$. Note that $\nabla \cdot \mathbf{u}=e^{-x}+e^{y} \neq 0$; the divergence-free condition on the flow was not needed for the 
preceding propositions. In this case, a particle located at $(x, y)$ moves according to the equations:

$$
\frac{d x}{d t}=1-e^{-x}, \quad \frac{d y}{d t}=e^{y},
$$

which can be solved explicitly. Suppose $P_{0}$ is such that its zero level set is the unit circle and $\nabla P_{0} \equiv 1$ on this level set as indicated in the figure below.

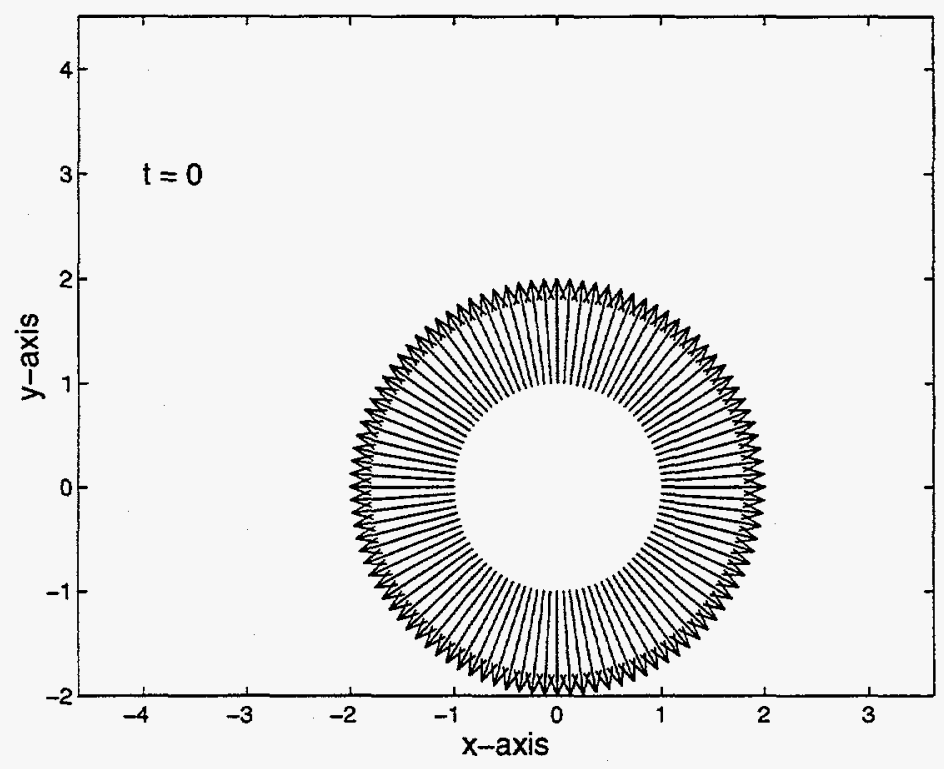

Figure 3.1: $\nabla P_{0}$ on the zero level set of $P_{0}$.

The equation of motion for $\nabla P_{t}$ is

$$
\frac{d \nabla P_{t}}{d t}=-\left(\begin{array}{cc}
e^{-x} & 0 \\
0 & e^{y}
\end{array}\right) \nabla P_{t} .
$$

The solution of this equation was found explicitly and graphed for times $t=0.25$ and $t=0.35$. The results are shown below.

\section{Remarks}

1. As expected, the vectors remain perpendicular to the set $\left\{\mathrm{x} \in \mathrm{R}^{2}: P_{t}(\mathbf{x})=0\right\}$.

2. From the matrix equation, it is clear that the magnitudes of the vectors $\nabla P_{t}$ always decrease. However, this effect is more pronounced in regions where the entries of the matrix are large, such as in the second quadrant. In regions where the entries are small, like the fourth quadrant, the vector magnitudes are reduced only very slightly. 

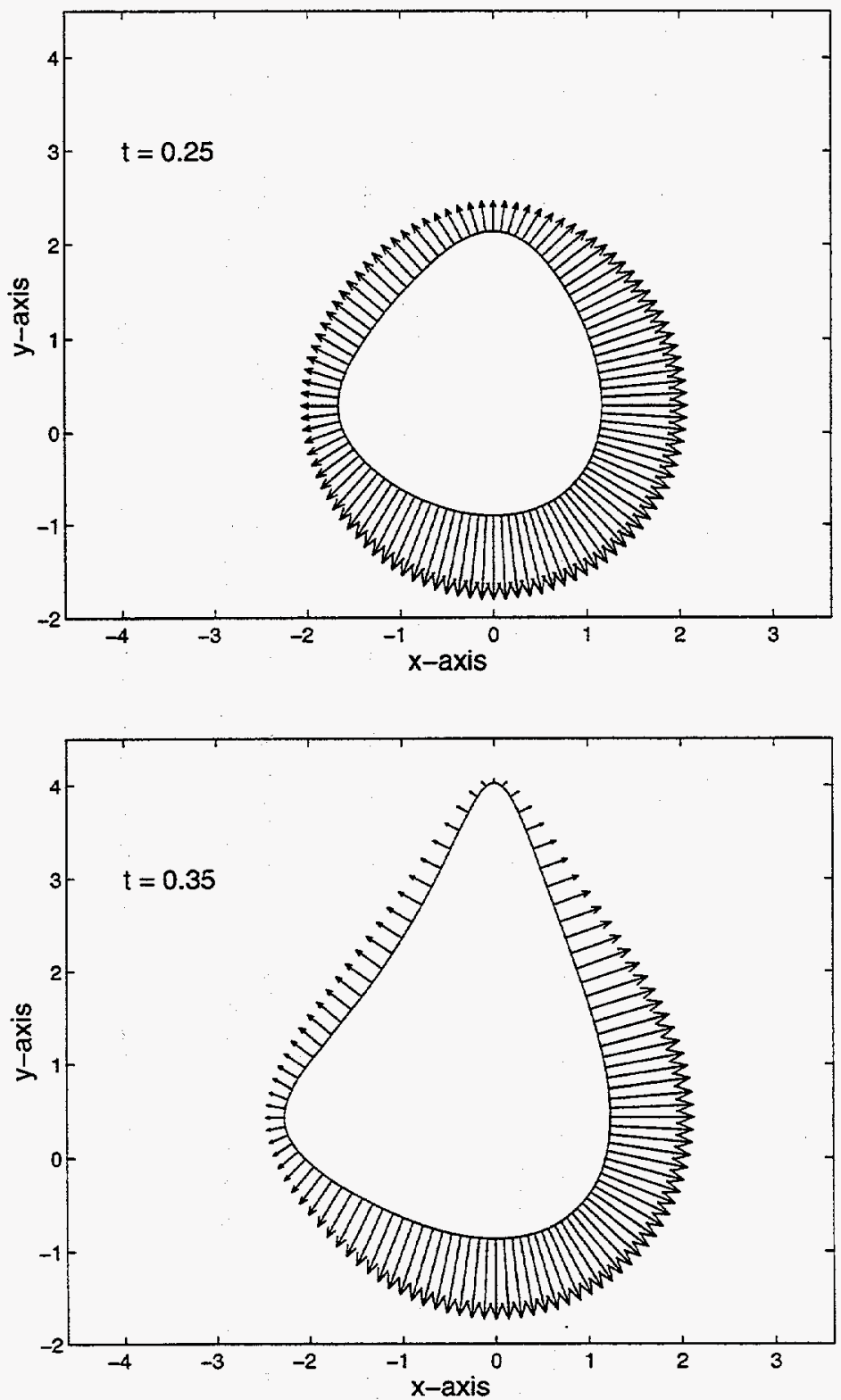

Figure 3.2: Solution at $t=0.25$ and $t=0.35$ 
3. In regions where the level set has been stretched by the flow, contiguous vectors are farther apart than in other regions. This will become an important issue when the connection between impulse methods and vortex methods is established.

4. The equations of motion for $\nabla P_{t}$ can be used to update vectors normal to a surface in situations where there is an underlying flow. For instance, motion under mean curvature requires computation of the unit normal vectors, which could be tracked by evolving them with the equations of motion for impulse and normalizing them after every step.

\subsection{Orthogonal Surfaces}

For rotational flows, the initial impulse field is not a gradient. However, proposition 5 can be extended to the case of impulse fields which are initially orthogonal to level sets of a given function but vary along these level sets.

Definition. Given a vector field $\mathbf{V} \in \mathrm{R}^{n}, a \mathbf{V}$-orthogonal surface is a surface (or curve in $\mathrm{R}^{2}$ ) on which $\mathbf{V}$ is perpendicular to the surface.

Proposition 6 Let $P_{0}(\alpha)=0$ implicitly define a surface $S_{0}$. Assume that on $S_{0}$ there is some function $H_{0}$ such that $\mathbf{m}$ is initially given by $\mathbf{m}(\alpha, 0)=H_{0}(\alpha) \nabla P_{0}(\alpha)$. Then,

$$
\begin{aligned}
\mathbf{m}(\mathbf{x}, t) & =H_{t}(\mathbf{x}) \nabla P_{t}(\mathbf{x})=H_{0}(\alpha) \nabla P_{t}(\mathbf{x}) \\
\xi(\mathbf{x}, t) & =\nabla H_{t}(\mathbf{x}) \times \nabla P_{t}(\mathbf{x})
\end{aligned}
$$

where $H_{t}(\mathbf{x})=H_{0} \circ \phi_{t}^{-1}(\mathbf{x})$ and $P_{t}(\mathbf{x})=P_{0} \circ \phi_{t}^{-1}(\mathbf{x})$, and the equations of motion for $\nabla H_{t}$, $\nabla P_{t}$ and $\mathbf{m}$ are identical. 
Proof: Let $H_{t}(\mathbf{x})=H_{0} \circ \phi_{t}^{-1}(\mathbf{x})$ and $P_{t}(\mathbf{x})=P_{0} \circ \phi_{t}^{-1}(\mathbf{x})$. Then for $\alpha \in S_{0}$ and $t \geq 0$

$$
\begin{aligned}
\mathbf{m}(\mathbf{x}, t) & =\left(\nabla \phi_{t}\right)^{-T} \mathbf{m}(\alpha, 0) \\
& =H_{0}(\alpha)\left(\nabla \phi_{t}\right)^{-T} \nabla P_{0}(\alpha) \\
& =H_{0}(\alpha) \nabla P_{t}(\mathbf{x})=H_{t}(\mathbf{x}) \nabla P_{t}(\mathbf{x})
\end{aligned}
$$

It follows that

$$
\begin{aligned}
\xi(\mathbf{x}, t) & =\nabla \times \mathbf{m}(\mathbf{x}, t)=\nabla \times\left[H_{t}(\mathbf{x}) \nabla P_{t}(\mathbf{x})\right] \\
& =\nabla H_{t}(\mathbf{x}) \times \nabla P_{t}(\mathbf{x}) .
\end{aligned}
$$

The equations of motion of $\nabla H_{t}, \nabla P_{t}$ and $\mathbf{m}$ are identical as established by proposition 5.

This proposition holds true for any smooth velocity field $\mathbf{u}$, as long as $\mathbf{m}$ evolves with the given equation of motion. If $\mathbf{m}$ is initially proportional to the gradient of some function $P_{0}$ on the $\mathbf{m}$-orthogonal surface $S_{0}$, then $\mathbf{m}$ will remain proportional to $\nabla P_{t}$. The proportionality factors, which are functions of space, will not change along particle paths. Consequently, the impulse will remain perpendicular to the evolving surface and the vorticity $\xi$ is the cross product of two gradients which evolve with the same equation.

\subsubsection{An Example in Two Dimensions}

Suppose an initial impulse field is defined on the unit circle by

$$
\mathbf{m}(\alpha, 0)=\frac{1}{2}[(\sin \theta+1] \alpha .
$$

This field can be represented as $\mathbf{m}(\alpha)=H_{0}(\alpha) \nabla P_{0}$, where $\nabla P_{0}=\alpha$ (i.e. radial unit vectors $)$ and $H_{0}=\frac{1}{2}[(\sin \theta+1]$. In subsequent chapters it will be noted that this initial setup is equivalent to defining vorticity on the unit circle. The initial impulse field is shown in figure 3.3. In the previous example, an arbitrary flow field was imposed to show the evolution of the gradient vectors. In this example, the flow will be the one induced by the impulse. The numerical method used here is the one introduced by Buttke [7], which will be explained in detail in the next chapter. The purpose of this example is to show the evolution of the impulse vectors and the gradient vectors, and to show that at any time $t$, the impulse field is given by $\mathbf{m}(\mathbf{x}, t)=H_{0}(\alpha) \nabla P_{t}(\mathbf{x})$. Figure 3.4 shows the gradient $\nabla P_{t}$ 


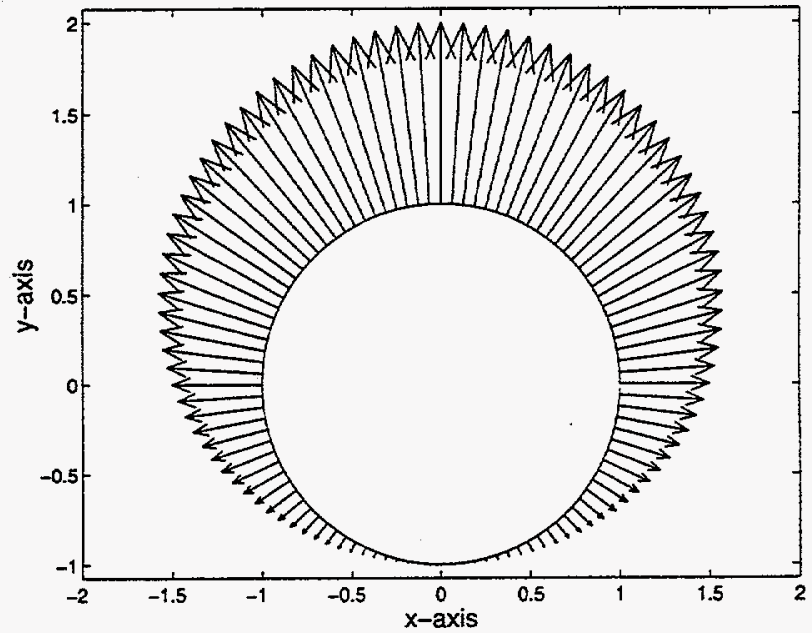

Figure 3.3: Initial impulse field.
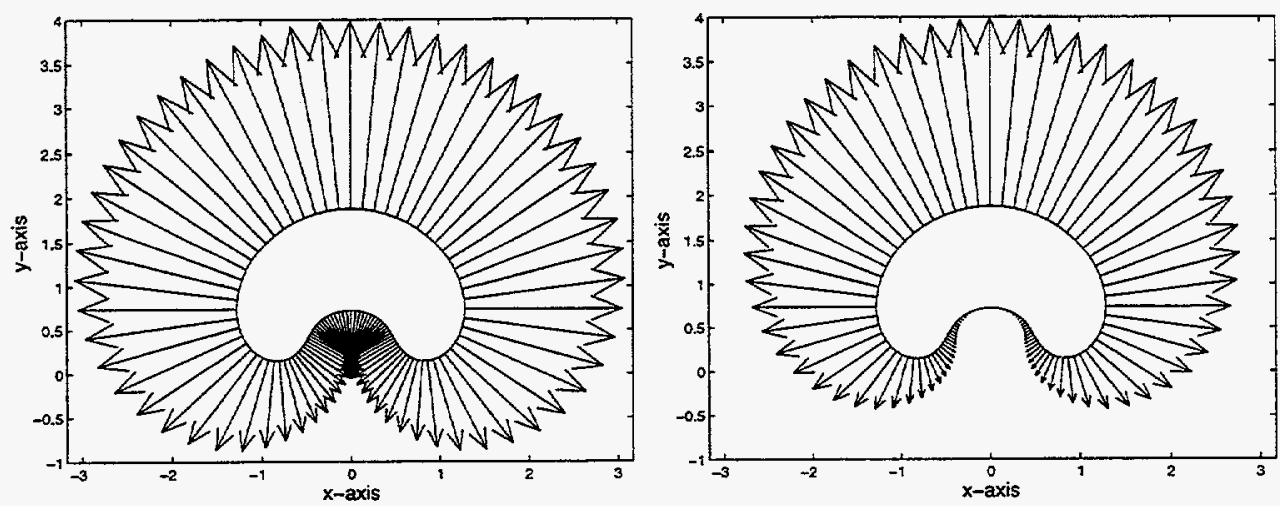

Figure 3.4: Plot of $\nabla P_{t}$ (left) and $\mathrm{m}$ (right) at time $t=6$. 
and the impulse at time $t=6$. According to proposition 6 , the ratio of vector lengths taken along the m-orthogonal surface must equal the function $H_{0}$. The vector lengths and the ratio $\left|\nabla P_{t}\right| /|\mathbf{m}|$ were computed and are shown below to coincide exactly with $H_{0}$.

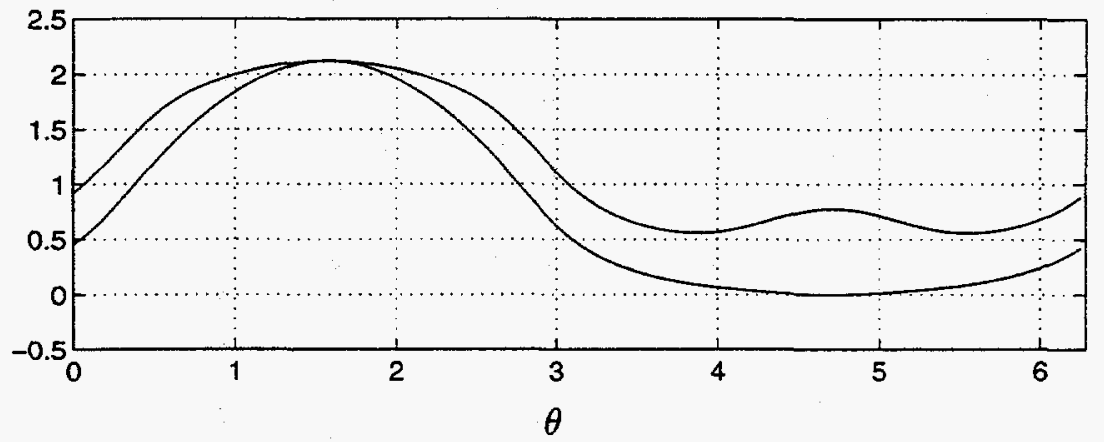

Figure 3.5: Plot of $\left|\nabla P_{t}\right|$ (top line) and $|\mathbf{m}|$ (bottom line) as functions of $\theta$.

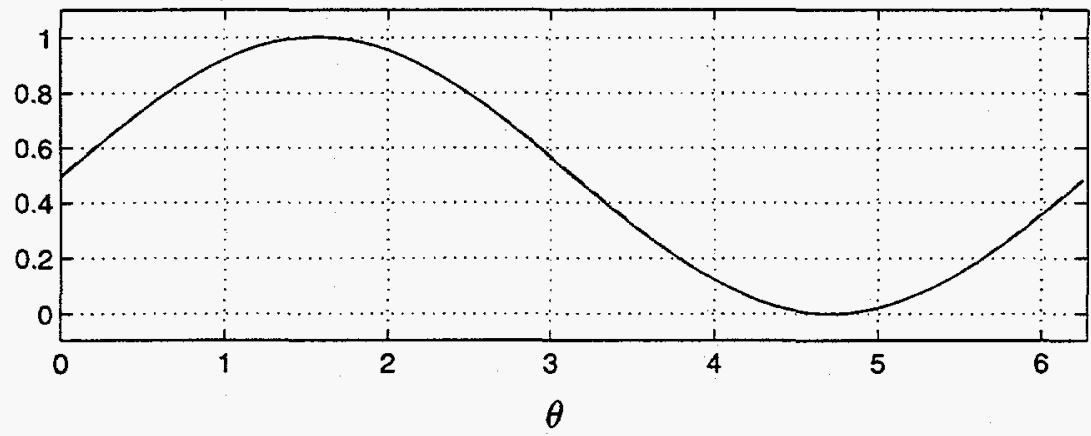

Figure 3.6: Plots of the ratio $\left|\nabla P_{t}\right| /|\mathbf{m}|$ and $(\sin (\theta)+1) / 2$.

\subsubsection{An Example in Three Dimensions}

Consider the following initial impulse field in cylindrical coordinates defined on the disc $r \leq 1$ and $z=0$

$$
\mathbf{m}(r, \theta, z)= \begin{cases}0.25(1-r) \hat{z}, & \text { on the disc } \\ 0, & \text { otherwise. }\end{cases}
$$

The disc is an m-orthogonal surface as illustrated in figure 3.7. Let the flow be the one induced by this impulse. 


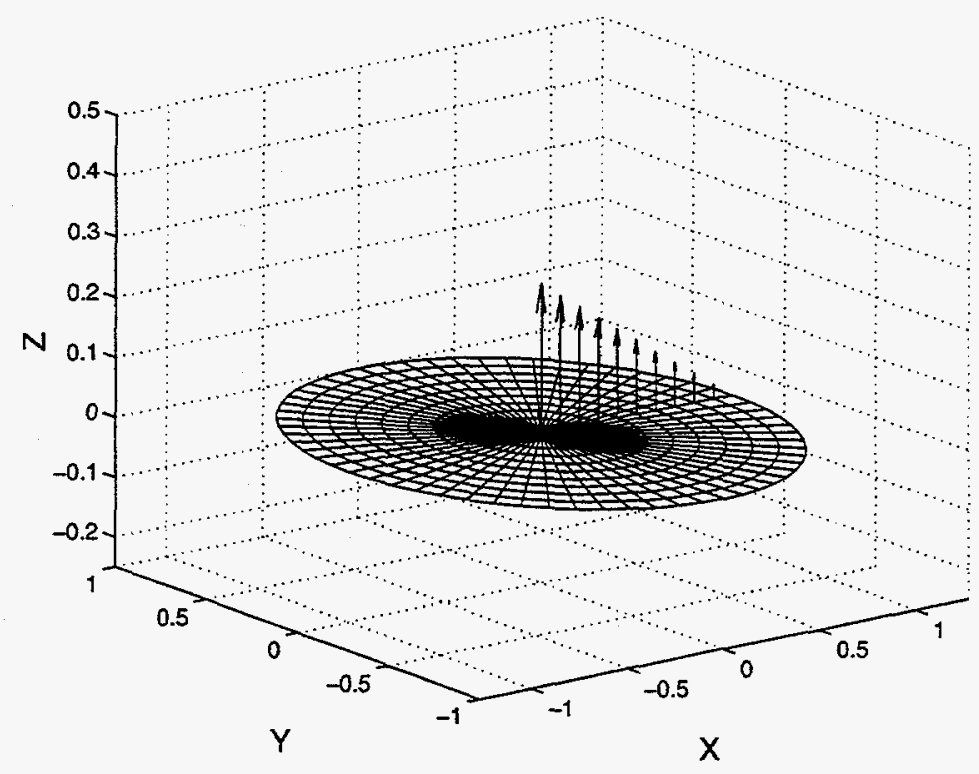

Figure 3.7: Initial impulse (radially symmetric).

As the flow evolves, the fluid moves around the outside of the disc and is ejected into the disc from below while pushing down on the edges. This causes the surface to swell up near the center of the disc, creating a bell-shaped surface. The solution, which is radially symmetric, is shown at time $t=2$ in figure 3.8. One can think of the disc as the zero level set of a function $P_{0}$ with unit gradient pointing upward. According to proposition 6 , the ratio $\left|\nabla P_{t}\right| /|\mathbf{m}|$ does not change along particle trajectories. Let $s$ be a parameter along the m-orthogonal surface such that $0 \leq s \leq 1, s=0$ at the center, and $s=1$ at the edge of the surface (so that $s=r$ is initially). The next figures show $\left|\nabla P_{t}\right|,|\mathbf{m}|$, and their rato as functions of $s$. Note that the ratio coincides with $H_{0}$.

When $S_{0}$ is the zero level set of a function $P_{0}$, then along flow lines $\nabla P_{t}$ evolves in such a way that it remains normal to the evolved level set. However, the area of the surface may change even when the flow is incompressible since the flow can accommodate larger areas by contracting in the direction normal to the surface. The next proposition points out that the growth of the area of an m-orthogonal surface is related to the growth of $|\mathbf{m}|$. Consider first the following definition:

Definition. The divergence of the flow $\mathbf{u}$ along a surface $S_{0}$ is $(\nabla \cdot \mathbf{u})$ restricted to $S_{0}$, and will be denoted by $\left.(\nabla \cdot \mathbf{u})\right|_{S_{0}}$. 


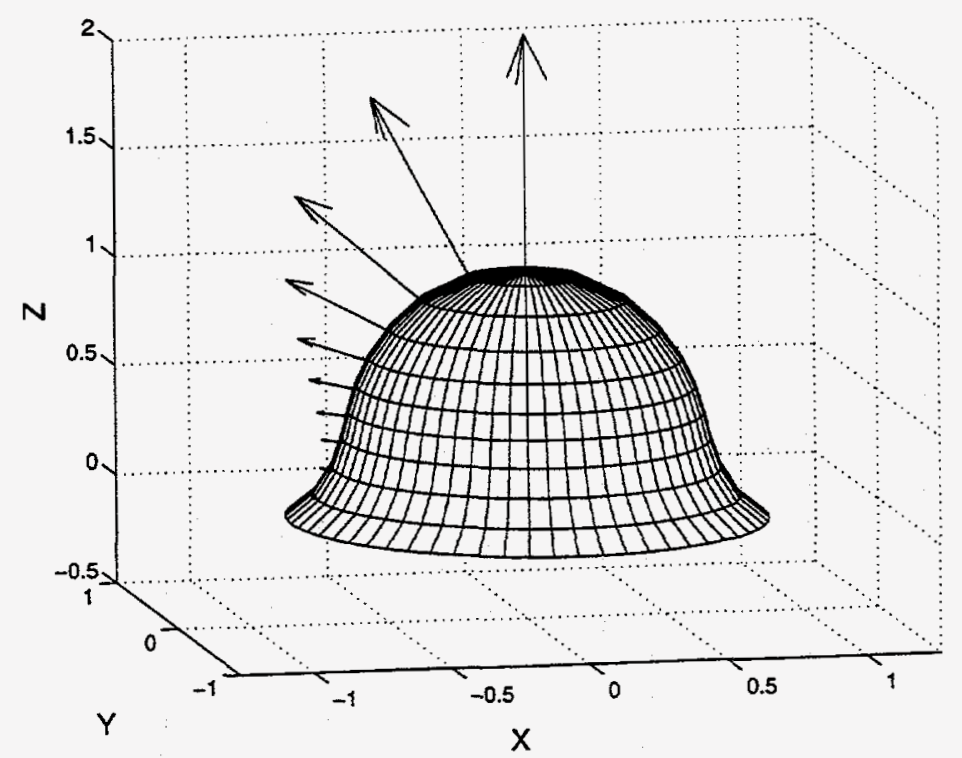

Figure 3.8: Impulse field at time $t=2$ (radially symmetric).

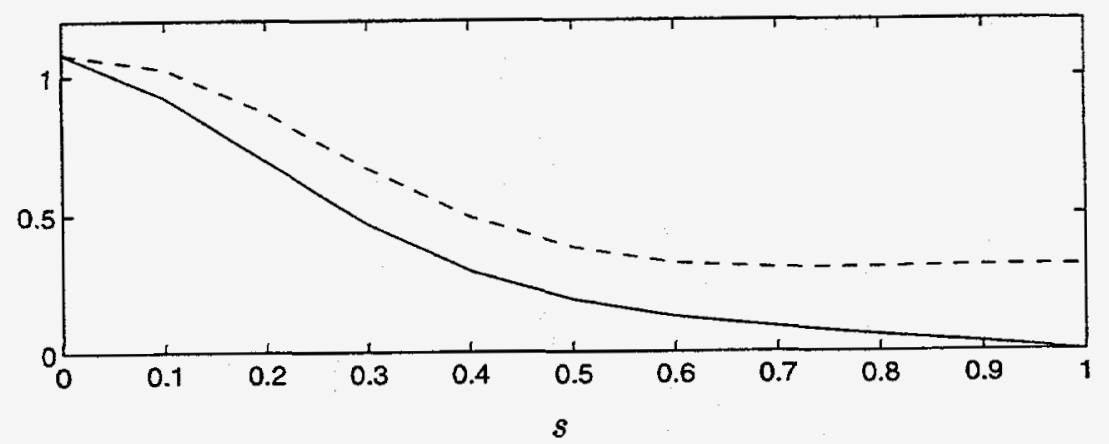

Figure 3.9: Plot of $\left|\nabla P_{t}\right|$ (dashed) and $|\mathbf{m}|$ (solid) as functions of $s$. 


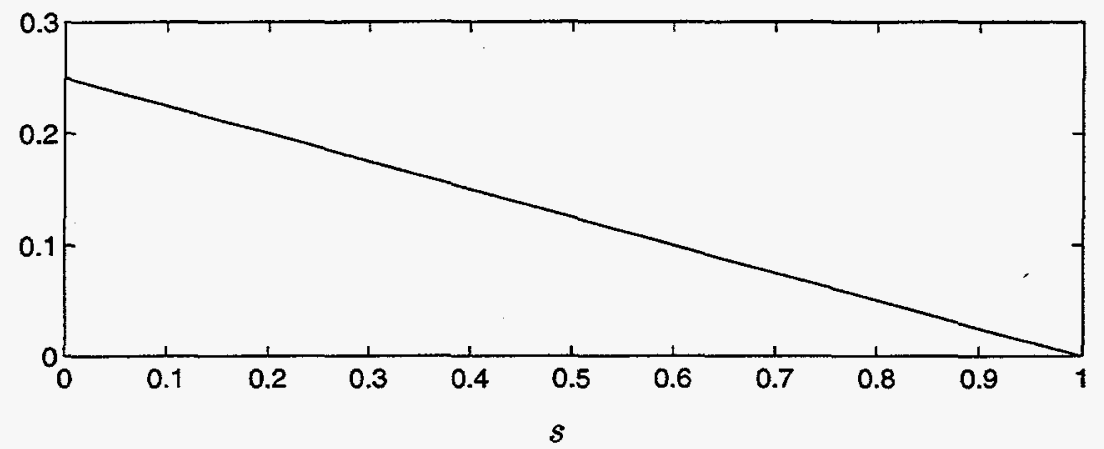

Figure 3.10: Plot of the ratio $\left|\nabla P_{t}\right| /|\mathbf{m}|$.

As an example consider the situation where $\mathbf{u}(x, y, z)=\left(u_{1}, u_{2}, u_{3}\right)^{T}$ and $S_{0}$ is the $x y$-plane. Then $\left.(\nabla \cdot \mathbf{u})\right|_{S_{0}}=\partial u_{1} / \partial x+\partial u_{2} / \partial y$.

Let $S_{0}$ be an $\mathbf{m}$-orthogonal surface and let $\Omega$ be a piece of $S_{0}$ such that $\mathbf{x}_{0} \in \Omega$. Suppose that the divergence of the flow restricted to $S_{0}$ is constant in $\Omega$. Then, if the surface area of $\Omega$ is denoted by $A$, the material derivative of $A$ is

$$
\frac{D A}{D t}=\int_{\partial \Omega} \mathbf{u} \cdot \hat{\mathbf{n}} d l=\left.\int_{\Omega}(\nabla \cdot \mathbf{u})\right|_{S_{0}} d S=\left.(\nabla \cdot \mathbf{u})\right|_{S_{0}} A,
$$

where $\hat{\mathbf{n}}$ is a unit vector on $S_{0}$ normal and outward to $\partial \Omega$.

Proposition 7 Let $S_{0}$ be an $\mathbf{m}$-orthogonal surface and fix $\mathbf{x}_{0} \in S_{0}$. Then for $\nabla \cdot \mathbf{u}=0$,

$$
\frac{D\left|\mathbf{m}\left(\mathbf{x}_{0}\right)\right|}{D t}=\left.(\nabla \cdot \mathbf{u})\right|_{S_{0}}\left|\mathbf{m}\left(\mathbf{x}_{0}\right)\right|
$$

and therefore $|\mathbf{m}|$ locally satisfies the same ordinary differential equation as the surface area of a small neighborhood of $\mathbf{x}_{0}$ on $S_{0}$.

Proof: Let $\mathbf{m}\left(\mathbf{x}_{0}\right) \neq 0$ and let $\hat{\mathbf{a}}$ be a fixed unit vector in the direction of $\mathbf{m}\left(\mathbf{x}_{0}\right)$ (i.e. normal to $S_{0}$ at $\mathbf{x}_{0}$ ), and let the flow $\mathbf{u}$ be incompressible. Then at $\mathbf{x}_{0}$,

$$
\begin{aligned}
\left.|\mathbf{m}|(\nabla \cdot \mathbf{u})\right|_{S_{0}} & =|\mathbf{m}|[\nabla \cdot \mathbf{u}-\hat{\mathbf{a}} \cdot \nabla(\mathbf{u} \cdot \hat{\mathbf{a}})] \\
& =-|\mathbf{m}|(\nabla \mathbf{u})^{T} \hat{\mathbf{a}} \cdot \hat{\mathbf{a}}=-(\nabla \mathbf{u})^{T} \mathbf{m} \cdot \frac{\mathbf{m}}{|\mathbf{m}|} \\
& =\frac{D \mathbf{m}}{D t} \cdot \frac{\mathbf{m}}{|\mathbf{m}|}=\frac{D|\mathbf{m}|}{D t}
\end{aligned}
$$


Comparing the result of proposition 7 with Eq. (3.1) one concludes that the relative growth of the area of an $\mathbf{m}$-orthogonal surface is equal to that of $|\mathbf{m}|$ in regions where $\left.(\nabla \cdot \mathbf{u})\right|_{S_{0}}$ can be considered constant. This fact will become important in the numerical method, where discrete impulse vectors carry elements of volume. Each volume element can be thought of as the area of a piece of an m-orthogonal surface multiplied by a thickness, where the length of each vector is proportional to the area it carries.

\subsubsection{An Example in Three Dimensions}

In this example, the vectors $\nabla P_{t}$ will be used instead of $\mathbf{m}$ to emphasize that the flow is not induced by the impulse but imposed externally. Consider the flow $\mathbf{u}(x, y, z)=$ $(x, y,-2 z)^{T}$. Let $S_{0}$ be the $x y$-plane, and let $\nabla P_{0} \equiv(0,0,1)^{T}$. Then the divergence of $\mathbf{u}$ restricted to $S_{0}$ is $\left.(\nabla \cdot u)\right|_{S_{0}}=\frac{\partial u_{1}}{\partial x}+\frac{\partial u_{2}}{\partial y}=1+1=2$. Additionally,

$$
\frac{D}{D t} \nabla P_{t}=-(\nabla \mathbf{u})^{T} \nabla P_{t}=-\left(\begin{array}{ccc}
1 & 0 & 0 \\
0 & 1 & 0 \\
0 & 0 & -2
\end{array}\right) \nabla P_{t}
$$

One can readily see that $\frac{D}{D t}\left|\nabla P_{t}\right|=2\left|\nabla P_{t}\right|$. On the other hand, a circular patch on $S_{0}$ of radius $r$, centered at the origin has area $A=\pi r^{2}$. Then,

$$
\frac{D A}{D t}=2 \pi r \frac{D r}{D t}=2 \pi(x, y, 0)^{T} \cdot(x, y, 0)^{T}=2 \pi r^{2}=2 A .
$$




\section{Chapter 4}

\section{A Lagrangian Numerical Method}

\subsection{Buttke's Method}

In [7], the impulse field is discretized by subdividing the domain into cells with the volume (or area) of the $j$-th cell equal to $A^{j}$, and by letting $\mathbf{m}^{j}=\mathbf{m}\left(\mathbf{x}^{j}\right) A^{j}$, where $\mathbf{x}^{j}$ is the center of the cell. Then an approximate impulse field is given by the sum

$$
\tilde{\mathbf{m}}(\mathbf{x})=\sum_{j=1}^{N} \mathbf{m}^{j}(t) f_{\delta}\left(\mathbf{x}-\mathbf{x}^{j}\right),
$$

where $N$ is the total number of cells and $f_{\delta}$ is a smooth function. For Eq. (4.1) to be a reasonable approximation to $\mathbf{m}(\mathbf{x})$, the function $f_{\delta}$ must approximate a Dirac delta function. In the theory of vortex methods, $f_{\delta}$ is called the cutoff function or the blob function and the subscript $\delta$ refers to the cutoff radius. Cutoff functions are chosen to satisfy certain conditions which guarantee small discretization errors due to the replacement of the Dirac delta by $f_{\delta}$ (see e.g. $[1,3,21,14,32,28]$ ). Typically, $f_{\delta}(\mathbf{x})=\delta^{-2} f_{1}(\mathbf{x} / \delta)$ in two dimensions and $f_{\delta}(\mathbf{x})=\delta^{-3} f_{1}(\mathbf{x} / \delta)$ in three dimensions, where the function $f_{1}$ satisfies the following conditions:

1. $\int f_{1}(\mathbf{x}) d \mathbf{x}=1$,

2. $\int x_{1}^{\beta_{1}} x_{2}^{\beta_{2}} x_{3}^{\beta_{3}} f_{1}(\mathbf{x}) d \mathbf{x}=0, \quad 0<\beta_{1}+\beta_{2}+\beta_{3}<k$,

3. $\int x_{1}^{\beta_{1}} x_{2}^{\beta_{2}} x_{3}^{\beta_{3}} f_{1}(\mathbf{x}) d \mathbf{x}<\infty, \quad \beta_{1}+\beta_{2}+\beta_{3}=k$.

Here, $k$ a fixed positive integer and $\beta_{i}$ 's are non-negative integers. A smooth function satisfying the conditions above causes discretization errors to be $O\left(\delta^{k}\right)$, and is therefore 
called a $k$-th order cutoff function. For a more detailed discussion of cutoff functions, see $[1,3,32,19]$. Choosing a cutoff function from vortex methods may seem reasonable; however, there is no a priori knowledge of whether this is a good choice or whether new functions should be designed. The next section will settle this question.

In order to arrive at a discretization of equations (2.14)-(2.15) given the approximation in Eq. (4.1), the fluid velocity at locations $\mathbf{x}^{j}$ due to $\tilde{\mathbf{m}}$ and the derivatives of this velocity must be computed. The procedure is described below for the case of Euler flow. The inclusion of the viscous term will be discussed in a later chapter. In this section, $n$ represents the spatial dimension $(n=2$ or 3 ).

\subsubsection{Step 1: The Particle Velocities}

Recall that if $\tilde{\mathbf{m}}=\mathbf{u}+\nabla \phi$, then $\mathbf{u}=\mathbb{P} \tilde{\mathbf{m}}$. The process of finding the velocity $\mathbf{u}$ in terms of $\tilde{\mathbf{m}}$ can be done exactly for a good choice of radially symmetric function $f_{\delta}$. First one takes the divergence of $\tilde{\mathbf{m}}=\mathbf{u}+\nabla \phi$ to obtain

$$
\Delta \phi=\nabla \cdot \tilde{\mathbf{m}}=\sum_{j=1}^{N} \mathbf{m}^{j} \cdot \nabla f_{\delta}\left(\mathbf{x}-\mathbf{x}^{j}\right) .
$$

Suppose that a function $\psi$ satisfies $\Delta \psi=f_{\delta}$, then one could write $\phi=\sum \mathbf{m}^{j} \cdot \nabla \psi$. Since $\psi$ depends only on $f_{\delta}, \psi$ can be found once and for all. For a radially symmetric cutoff function, the equation for $\psi$ can be written in polar coordinates as

$$
r^{1-n}\left(r^{n-1} \psi_{r}\right)_{r}=f_{\delta}(r)
$$

where the subscript $r$ represents a partial derivative with respect to $r$. Ultimately only $\nabla \psi$ is needed, not $\psi$ itself. Thus the last equation can be solved for $\psi_{r}$ to obtain

$$
\psi_{r}(r)=r^{1-n} \int_{0}^{r} q^{n-1} f_{\delta}(q) d q=\frac{F(r)}{\pi(2 r)^{n-1}}
$$

where $F(r)=\int_{|\mathbf{x}| \leq r} f_{\delta}(|\mathbf{x}|) d \mathbf{x}$ is called the shape factor, and depends only on the cutoff function.

Since $\phi=\sum \mathrm{m}^{j} \cdot \nabla \psi$, an expression for $\phi$ can now be found. After differentiating the resulting expression, a formula for $\mathbf{u}=\tilde{\mathbf{m}}-\nabla \phi$ is derived. The final result in terms of the shape factor is

$$
\mathbf{u}(\mathbf{x})=\frac{1}{\pi 2^{n-1}} \sum_{j=1}^{N} \mathbf{m}^{j}\left[\frac{r F^{\prime}(r)-F(r)}{r^{n}}\right]-\hat{\mathbf{x}}^{j}\left(\mathbf{m}^{j} \cdot \hat{\mathbf{x}}^{j}\right)\left[\frac{r F^{\prime}(r)-n F(r)}{r^{n}}\right],
$$

where $\hat{\mathbf{x}}^{j}=\left(\mathbf{x}-\mathbf{x}^{j}\right) / r$ and $r=\left|\mathbf{x}-\mathbf{x}^{j}\right|$. 


\subsubsection{Step 2: The Rate of Change of Impulse}

The impulse carried by the particles must be updated by an equation approximating Eq. (2.15). Differentiation of the expression for $\mathbf{u}(\mathbf{x})$ leads to the matrix $\nabla \mathbf{u}$, whose $k$-th column is given by

$$
\begin{aligned}
\frac{\partial \mathbf{u}(\mathbf{x})}{\partial x_{k}} & =\frac{1}{\pi 2^{n-1}} \sum_{j=1}^{N} \mathbf{m}^{j} \hat{x}_{k}^{j}\left[\frac{r^{2} F^{\prime \prime}-n r F^{\prime}+n F}{r^{n+1}}\right]-m_{k}^{j} \hat{\mathbf{x}}^{j}\left[\frac{r F^{\prime}-n F}{r^{n+1}}\right] \\
& -\hat{\mathbf{e}}^{k}\left(\mathbf{m}^{j} \cdot \hat{\mathbf{x}}^{j}\right)\left[\frac{r F^{\prime}-n F}{r^{n+1}}\right]-\hat{x}_{k}^{j} \hat{\mathbf{x}}^{j}\left(\mathbf{m}^{j} \cdot \hat{\mathbf{x}}^{j}\right)\left[\frac{r^{2} F^{\prime \prime}-(2 n+1) r F^{\prime}+n(n+2) F}{r^{n+1}}\right]
\end{aligned}
$$

where $\hat{\mathbf{x}}^{j}=\left(\mathbf{x}-\mathbf{x}^{j}\right) / r, r=\left|\mathbf{x}-\mathbf{x}^{j}\right|$, $\hat{\mathbf{e}}^{k}$ is the unit vector in the $k$-th direction, and all subscripts represent vector components.

The equations of motion for the particle locations and the impulse strengths are

$$
\begin{aligned}
\frac{d \mathbf{x}^{j}}{d t} & =\mathbf{u}\left(\mathbf{x}^{j}\right) \\
\frac{d \mathbf{m}^{j}}{d t} & =-(\nabla \mathbf{u})^{T} \mathbf{m}^{j}
\end{aligned}
$$

where $\mathbf{u}$ and the entries of $(\nabla \mathbf{u})$ are given by Eq. (4.2) and (4.3) respectively. From a computational point of view, these equations are written in a convenient form since the expressions in square brackets contain the smoothing effect of the cutoff function and can be found once and for all.

\subsection{The Hamiltonian Structure of Impulse Flow}

Eq. (4.4)-(4.5) form a discrete Hamiltonian system. The last part of chapter 2 shows the Hamiltonian from which the equation of motion for impulse is derived, as well as other known invariants of incompressible Euler flow. The discrete versions of these invariants are given now. The discrete Hamiltonian is

$$
H=\frac{1}{2} \sum_{j=1}^{N} \mathbf{u}\left(\mathbf{x}^{j}\right) \cdot \mathbf{m}^{j},
$$

which is initially an approximation of the continuous Hamiltonian Eq. (2.16). The total impulse, angular momentum and helicity density are $[7,8]$

$$
\mathbf{I}=\sum_{j=1}^{N} \mathbf{m}^{j}, \quad \sum_{j=1}^{N} \mathbf{x}^{j} \times \mathbf{m}^{j}, \quad \xi^{i} \cdot \mathbf{m}^{i} .
$$

One can show the invariance of all these quantities by using Eq. (4.4)-(4.5). 


\subsection{Comments About the Numerical Method}

In a vortex method, one typically approximates the vorticity by the sum $\tilde{\xi}(\mathbf{x})=$ $\sum \xi^{j} f_{\delta}\left(\left|\mathbf{x}-\mathbf{x}^{j}\right|\right)$. The velocity field induced by this vorticity can be written formally as

$$
\mathbf{u}=-\nabla \times \Delta^{-1} \bar{\xi}
$$

where $\Delta^{-1}$ represents the inverse of the Laplacian operator. If impulse is approximated by the sum in Eq. (4.1), the velocity field of Eq. (4.2) is

$$
\mathbf{u}=\tilde{\mathbf{m}}-\nabla\left(\nabla \cdot \Delta^{-1} \tilde{\mathbf{m}}\right)
$$

The additional differentiation of the approximate field required in the impulse method points out that the impulse method is more singular than the vortex method. The cutoff function is designed to smoothly eliminate the singularity in the velocity field.

A Lagrangian numerical method based on impulse is possible because when the vorticity is concentrated in a bounded region, the impulse can be defined with compact support. This is the case because outside the support of vorticity the fluid velocity is a gradient (i.e. the flow is potential), and the function $\phi$ can be chosen so that its gradient cancels the velocity outside a set containing the support of vorticity. In general, the support of impulse contains, but does not equal, the support of vorticity. One can see this by considering a two-dimensional flow in which vorticity is confined to two disjoint and bounded regions with net vorticity +1 and -1 respectively. Let $\Omega$ be an open set containing only the first region and consider the circulation around the boundary of $\Omega$. The circulation is $\int_{\partial \Omega} \mathbf{m} \cdot d \mathbf{l}=\int_{\Omega} \xi \cdot d \mathbf{A}=+1$, and the impulse cannot vanish identically on the boundary of $\Omega$. A similar example in three dimensions is found in [7]. Impulse must be defined in a larger domain than vorticity.

A simple calculation shows that the vorticity field induced by impulse, $\xi(\mathbf{x})=$ $\nabla \times \sum_{j} \mathbf{m}^{j} f_{\delta}\left(\mathbf{x}-\mathbf{x}^{j}\right)$, automatically satisfies the condition $\nabla \cdot \xi=0$. This important property is not necessarily shared by other methods [32].

As pointed out in the previous section, the impulse equations are the canonical Hamiltonian formulation of fluid flow in any space dimensions $[24,7]$. In practice, expressions for invariants associated with the Hamiltonian system of equations can be used to determine parameters of the impulse method and to monitor the computation. Also, there is a large amount of mathematical machinery applicable to Hamiltonian systems which can be exploited in the impulse method. 
Consider the three-dimensional case of the velocity field in Eq. (4.2). Suppose the cutoff function $f_{\delta}(\mathbf{x})$ has support in a ball of radius $\delta$ and that $\left|\mathbf{x}-\mathbf{x}^{j}\right|>\delta$ for all $j$. Then Eq. (4.2) reduces to

$$
\mathbf{u}(\mathbf{x})=\frac{1}{4 \pi} \sum_{j=1}^{N} \frac{-\mathbf{m}^{j}+3 \hat{\mathbf{x}}^{j}\left(\mathbf{m}^{j} \cdot \hat{\mathbf{x}}^{j}\right)}{r^{3}}
$$

which is the flow induced by dipoles (see [22] \$5.6). A complete connection between the velocity field due to an impulse vector and that due to a vortex dipole will be made later in this chapter.

\subsection{Discrete Impulse and Vortex Blobs}

Suppose that there is a single blob of impulse at the origin. The velocity at an arbitrary point $\mathbf{x}$ due to the impulse is

$$
\mathbf{u}(\mathbf{x})=\mathbf{m} f_{\delta}(|\mathbf{x}|)-(\mathbf{m} \cdot \nabla) \nabla \psi(|\mathbf{x}|), \quad \text { where } \Delta \psi=f_{\delta}
$$

Define the Green's function, $G$, as the function with the property that if $\Delta \zeta=-f_{\delta}$ in free space, then $\zeta=G * f_{\delta}$, where * denotes a convolution. In two dimensions $G(\mathbf{x})=\frac{1}{2 \pi} \log |\mathbf{x}|$, while in three dimensions $G(\mathbf{x})=-\frac{1}{4 \pi}|\mathbf{x}|^{-1}$, both for $|\mathbf{x}| \neq 0$. In terms of the Green's function, $\psi$ can be written as $\psi=-G * f_{\delta}$. Using the identity $(\mathbf{F} \cdot \mathbf{H}) \mathbf{G}=\mathbf{F} \times(\mathbf{G} \times \mathbf{H})+$ $(\mathbf{F} \cdot \mathbf{G}) \mathbf{H}$ one obtains for $|\mathbf{x}| \neq 0$,

$$
\begin{aligned}
\mathbf{u}(\mathbf{x}) & =\mathbf{m} f_{\delta}+(\mathbf{m} \cdot \nabla)\left(\nabla G * f_{\delta}\right)=\mathbf{m} f_{\delta}+\nabla G *\left(\mathbf{m} \cdot \nabla f_{\delta}\right) \\
& =\mathbf{m} f_{\delta}+\mathbf{m} \sum_{i=1}^{n} \frac{\partial G}{\partial x_{i}} * \frac{\partial f_{\delta}}{\partial x_{i}}+\int \nabla f_{\delta}(\mathbf{z}) \times(\nabla G(\mathbf{x}-\mathbf{z}) \times \mathbf{m}) d \mathbf{z}
\end{aligned}
$$

If one defines the operator $K(\mathbf{x})=\nabla G(\mathbf{x}) \times$, the expression for velocity can be written as

$$
\begin{aligned}
\mathbf{u}(\mathbf{x}) & =\mathbf{m} f_{\delta}+\mathbf{m} \nabla \cdot\left(\nabla G * f_{\delta}\right)+\int \nabla f_{\delta}(\mathbf{z}) \times K(\mathbf{x}-\mathbf{z}) \mathbf{m} d \mathbf{z} \\
& =\mathbf{m} f_{\delta}-\mathbf{m} \Delta\left(-G * f_{\delta}\right)+\nabla \times \int K(\mathbf{x}-\mathbf{z}) \mathbf{m} f_{\delta}(\mathbf{z}) d \mathbf{z} \\
& =\nabla \times\left(K * f_{\delta}\right) \mathbf{m}=K *\left(\nabla \times \mathbf{m} f_{\delta}\right)
\end{aligned}
$$

which is the velocity field due to a vorticity distribution $\xi(\mathbf{x})=\nabla \times \mathbf{m} f_{\delta}(\mathbf{x})$. It is therefore important to analyze in detail the vorticity induced by impulse vectors and to establish connections with vortex methods. 


\subsection{Two-dimensional Impulse and Vortex Dipoles}

The previous calculation shows that the velocity field due to one impulse blob is the same as the velocity induced by the vorticity $\xi(\mathbf{x})=\nabla \times \mathbf{m} f_{\delta}(\mathbf{x})$. This section presents an analysis of this vorticity in two dimensions and its connection to vortex blobs.

Given an approximate impulse field consisting of one blob located at the origin, $\tilde{\mathbf{m}}(\mathbf{x})=\mathbf{m}^{0}(t) f_{\delta}(\mathbf{x})$, one can compute the curl of $\tilde{\mathbf{m}}(\mathbf{x})$ to get

$$
\xi(\mathbf{x})=\nabla \times \mathbf{m}^{0} f_{\delta}(\mathbf{x})=\nabla f_{\delta}(\mathbf{x}) \times \mathbf{m}^{0} .
$$

Let the impulse strength be given by $\mathbf{m}^{0}(t)=\left(m_{1}, m_{2}, 0\right)^{T}$ and $\xi=(0,0, \omega)^{T}$. Then Eq. (4.6) implies

$$
\omega(\mathbf{x})=\nabla f_{\delta}(\mathbf{x}) \cdot\left(m_{2},-m_{1}, 0\right)^{T}=\nabla f_{\delta}(\mathbf{x}) \cdot\left(\mathbf{m}^{0} \times \hat{\mathbf{z}}\right)
$$

where $\hat{\mathbf{z}}$ is the unit vector normal to the plane. The vector $\left(\mathbf{m}^{0} \times \hat{\mathbf{z}}\right)$ has magnitude $\left|\mathbf{m}^{0}\right|$ and is perpendicular to $\mathbf{m}^{0}$. Thus, one can interpret the vorticity as $\left|\mathbf{m}^{0}\right|$ times the derivative of the cutoff function $f_{\delta}$ in the direction of $\left(\mathbf{m}^{0} \times \hat{\mathbf{z}}\right)$. Let $\mathbf{h}$ represent a vector in the direction of $\left(\mathbf{m}^{0} \times \hat{\mathbf{z}}\right)$ and let $|\mathbf{h}|=h$. Then, the vorticity may be written as

$$
\begin{aligned}
\omega(\mathbf{x}) & =\left|\mathbf{m}^{0}\right| \lim _{h \rightarrow 0} \frac{f_{\delta}(\mathbf{x}+\mathbf{h})-f_{\delta}(\mathbf{x}-\mathbf{h})}{2 h} \\
& =\lim _{h \rightarrow 0} \omega^{0}\left[f_{\delta}(\mathbf{x}+\mathbf{h})-f_{\delta}(\mathbf{x}-\mathbf{h})\right],
\end{aligned}
$$

where $\omega^{0}=\left|\mathbf{m}^{0}\right| / 2 h$. This expression shows that the vorticity induced by each impulse vector is that of a vortex dipole. A vortex dipole is the limit of two vortex blobs of equal and opposite strength as their separation goes to zero. Since the vortex strengths depend on the separation but $2 h \omega^{0}=\left|\mathbf{m}^{0}\right|$ is constant in the limit process, this limit is taken in a way that maintains a constant dipole moment equal to $-2 \mathbf{h} \omega^{0}=\left(\hat{\mathbf{z}} \times \mathbf{m}^{0}\right)$.

In two dimensions, a discrete impulse vector induces the same velocity field as a vortex dipole with dipole moment prescribed by the impulse vector. If one neglects the limit process in the expression for vorticity, and simply places two vortex blobs along the line normal to $\mathbf{m}^{0}$ at a distance $2 h$ with strengths $\omega^{0}= \pm\left|\mathbf{m}^{0}\right| / 2 h$, the new vorticity $\omega(\mathbf{x})$ may be written as

$$
\omega(\mathbf{x})=\omega^{0}\left[f_{\delta}(\mathbf{x}+\mathbf{h})-f_{\delta}(\mathbf{x}-\mathbf{h})\right]
$$

There are several key observations that one can make about this expression. One way to gain intuition about impulse variables is to think of each vector as approximately a vortex pair 


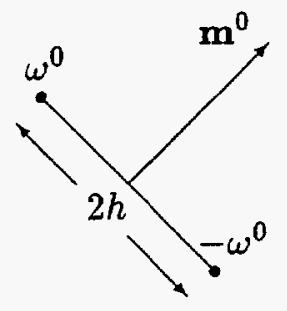

Figure 4.1: Vortex Pair Approximation to Impulse Vector.

in the sense explained above. The approximation is more accurate as the two vortices are placed closer together near the base of the impulse vector. It is clear that Eq. (4.7) comes from replacing the derivative in expression (4.6) by a centered-difference approximation; thus the error in this replacement is $O\left(h^{2}\right)$. One of the most important observations is that the two vortex blobs in the formula for $\bar{\omega}(\mathbf{x})$ use the same cutoff function as the impulse blob. This allows one to export the role of the cutoff function in the accuracy of vortex methods into impulse methods, and validates the use of these cutoff functions.

The preceding discussion points to the importance of curves which are locally perpendicular to the impulse vectors. These curves are the m-orthogonal surfaces discussed in the previous chapter. Since impulse is a collection of vortex dipoles along the m-orthogonal curve, the flow represented by the impulse can be approximated by the flow due to an appropriate vorticity distribution along the curve. If there is vorticity in a strip surrounding the curve such that the net vorticity in the strip is zero, then the velocity due to the vorticity can be written as an line integral along the curve and the velocity due to impulse vectors which represent the same flow is a discretization of this integral. This is the subject of the next section.

\subsection{The Velocity Field}

Suppose that the vorticity is $\xi=(0,0, \omega)^{T}$, where the support of $\omega(\mathbf{x})$ is the strip in the figure below. Let the dotted curve have parametric representation $\mathbf{x}(s)$, where the arclength parameter $s$ is zero at the left end of the curve and $s=B$ at the right end. Let the thickness of the strip be $h$. Assume that the vorticity varies along the curve but that the strip is narrow enough that $\omega(\mathbf{x})$ can be considered constant in the transversal direction. 


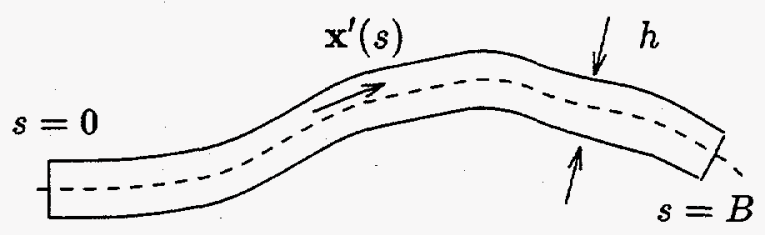

Figure 4.2: Strip in which vorticity is concentrated. The strip is assumed to have thickness $h$ and be parametrized by $s$.

Assume also that the net vorticity is zero and define $\Gamma(\mathbf{x}(s))=\int_{0}^{s} \omega(\mathbf{x}(q)) d q$. From this definition it is clear that $\Gamma(\mathbf{x}(0))=0$ and that $\Gamma(\mathbf{x}(B))=\int_{0}^{B} \omega(\mathbf{x}(q)) d q=0$ by assumption.

The velocity induced by the vorticity is

$$
\mathbf{u}(\mathbf{x})=K * \omega=\int K(\mathbf{x}-\mathbf{a}) \omega(\mathbf{a}) d \mathbf{a},
$$

where $K(x, y)=\frac{1}{2 \pi}(-y, x) / r^{2}$ is known as the vortex kernel, and $r^{2}=x^{2}+y^{2}$. In vortex methods, one generally uses a smoothed vortex kernel of the type $K_{\delta}(x, y)=K(x, y) F(r)$, where $F(r)$ is the shape factor defined at the beginning of this chapter. Then the velocity field due to the vorticity is approximated by $\mathbf{u}_{\delta}=K_{\delta} * \omega$. In the case at hand,

$$
\mathbf{u}_{\delta}(\mathbf{x})=h \int_{0}^{B} \omega(\mathbf{x}(s)) K_{\delta}(\mathbf{x}-\mathbf{x}(s)) d s=h \int_{0}^{B}\left[\frac{d}{d s} \Gamma(\mathbf{x}(s))\right] K_{\delta}(\mathbf{x}-\mathbf{x}(s)) d s .
$$

Since $\Gamma$ vanishes at both ends of the curve, one can integrate by parts to obtain

$$
\mathbf{u}_{\delta}(\mathbf{x})=-h \int_{0}^{B} \Gamma(\mathbf{x}(s))\left[\frac{d}{d s} K_{\delta}(\mathbf{x}-\mathbf{x}(s))\right] d s
$$

Note that $\frac{d}{d s} K_{\delta}(\mathbf{x}-\mathbf{x}(s))=-\left(\nabla K_{\delta}\right) \mathbf{x}^{\prime}(s)$, where $\mathbf{x}^{\prime}(s)$ is the unit vector tangent to the curve in the direction of increasing $s$. One can discretize the integral above by letting $\left\{\mathbf{x}^{j}\right\}_{j=1}^{N}$ be a set of points along the curve with spacing $(\Delta s)^{j}=\mathbf{x}^{j+1}-\mathbf{x}^{j}$. If the unit tangent vector at $\mathbf{x}^{j}$ is denoted by $\mathbf{x}^{\prime j}$, the discretization of the integral gives

$$
\overline{\mathbf{u}}(\mathbf{x})=\sum_{j=1}^{N} h(\Delta s)^{j} \Gamma\left(\mathbf{x}^{j}\right)\left(\nabla K_{\delta}\left(\mathbf{x}-\mathbf{x}^{j}\right)\right) \mathbf{x}^{i j}
$$

This is exactly the velocity field induced by impulse vectors placed along the curve and normal to it. To see this, differentiate $K_{\delta}$ to get

$$
2 \pi\left(\nabla K_{\delta}\right)(\mathbf{x})=\left(\begin{array}{ll}
-x y & -y^{2} \\
x^{2} & x y
\end{array}\right)\left(\frac{F(r)}{r^{2}}\right)^{\prime} \frac{1}{r}+\left(\begin{array}{ll}
0 & -1 \\
1 & 0
\end{array}\right) \frac{F(r)}{r^{2}} .
$$


Now, use the equalities $x^{2}=r^{2}-y^{2}$ and $-y^{2}=x^{2}-r^{2}$ in the first term to get

$$
\begin{aligned}
2 \pi\left(\nabla K_{\delta}\right)(\mathbf{x}) & =\left(\begin{array}{ll}
-x y & x^{2} \\
-y^{2} & x y
\end{array}\right)\left(\frac{F(r)}{r^{2}}\right)^{\prime} \frac{1}{r}+\left(\begin{array}{ll}
0 & -1 \\
1 & 0
\end{array}\right)\left[\left(\frac{F(r)}{r^{2}}\right)^{\prime} r+\frac{F(r)}{r^{2}}\right] \\
& =\left(\begin{array}{ll}
-x y & x^{2} \\
-y^{2} & x y
\end{array}\right)\left[\frac{r F^{\prime}-2 F}{r^{4}}\right]+\left(\begin{array}{ll}
0 & -1 \\
1 & 0
\end{array}\right)\left[\frac{r F^{\prime}-F}{r^{2}}\right] .
\end{aligned}
$$

Then

$$
2 \pi\left(\nabla K_{\delta}\left(\mathbf{x}-\mathbf{x}^{j}\right)\right) \mathbf{x}^{\prime j}=-\left(\left(\mathbf{x}-\mathbf{x}^{j}\right) \cdot\left(\hat{\mathbf{z}} \times \mathbf{x}^{\prime j}\right)\right)\left(\mathbf{x}-\mathbf{x}^{j}\right)\left[\frac{r F^{\prime}-2 F}{r^{4}}\right]+\left(\hat{\mathbf{z}} \times \mathbf{x}^{\prime j}\right)\left[\frac{r F^{\prime}-F}{r^{2}}\right]
$$

where $\hat{\mathbf{z}}$ is the unit vector normal to the plane and $r=\left|\mathbf{x}-\mathbf{x}^{j}\right|$. Finally, let $\mathbf{m}^{j}=$ $h(\Delta s)^{j} \Gamma\left(\mathbf{x}^{j}\right)\left(\hat{\mathbf{z}} \times \mathbf{x}^{i j}\right)$ be vectors perpendicular to the curve, and let $\hat{\mathbf{x}}^{j}=\left(\mathbf{x}-\mathbf{x}^{j}\right) / r$ in order to simplify the notation. Then the velocity can be written as

$$
\tilde{\mathbf{u}}(\mathbf{x})=\frac{1}{2 \pi} \sum_{j=1}^{N}-\left(\hat{\mathbf{x}}^{j} \cdot \mathbf{m}^{j}\right) \hat{\mathbf{x}}^{j}\left[\frac{r F^{\prime}-2 F}{r^{2}}\right]+\mathbf{m}^{j}\left[\frac{r F^{\prime}-F}{r^{2}}\right] .
$$

This equation is identical to Eq. (4.2).

In practice; one discretizes impulse by identifying many strips in which the vectors can be defined. For example consider the problem of two vortex patches of constant vorticity $+\omega$ and $-\omega$ as shown in figure 4.3. Impulse vectors can be defined along horizontal strips as shown in the figure. If $\mathbf{m}=\left(0, m_{2}\right)$, then $m_{2}(x, y)=\int_{-\infty}^{x} \omega(\zeta, y) d \zeta$.

\subsection{An Example}

Consider the initial vorticity defined in the unit disc, given in polar coordinates by $\omega(r, \theta)=-30 r^{2}(r-1)^{2} \cos \theta$. This vorticity is antisymmetric about the $y$-axis and can be thought of as a large version of a smooth vortex dipole. As described in [7], this flow can be represented by the impulse field $\mathbf{m}=\left(m_{1}, m_{2}\right)$, where $m_{1} \equiv \mathbf{0}$ and $m_{2}(r, \theta)=$ $1-10 r^{3}+15 r^{4}-6 r^{5}$. Horizontal lines are m-orthogonal surfaces. As in [7], the initial parameters are square cells of side $h=0.05$ and cutoff function $f_{\delta}(r)=\delta^{-2} f_{1}(r / \delta)$, where $f_{1}(r)=7 / 2 \pi\left(1-10 r^{3}+15 r^{4}-6 r^{5}\right)$ and $\delta=0.4$. Figures 4.4-4.6 show the evolution of some of these surfaces and the impulse field defined on one of them for time between 0 and 3. The magnitudes of the vectors have been multiplied by a fixed scalar to make them more visible and only half of the surfaces used are shown. 

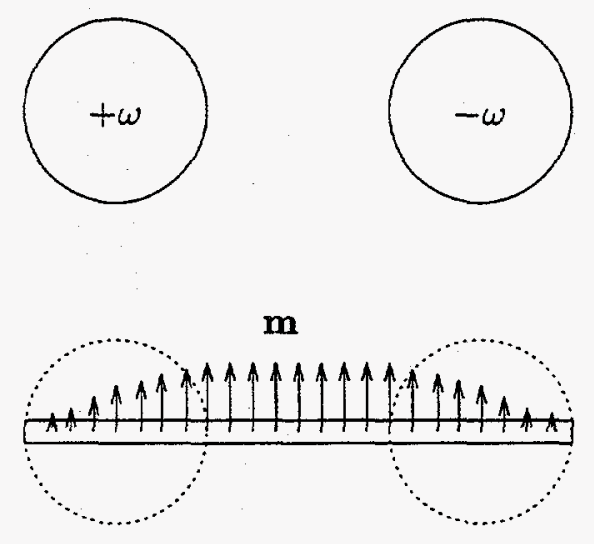

Figure 4.3: Two patches of constant vorticity (top). The bottom picture shows the corresponding impulse field defined on a horizontal strip.

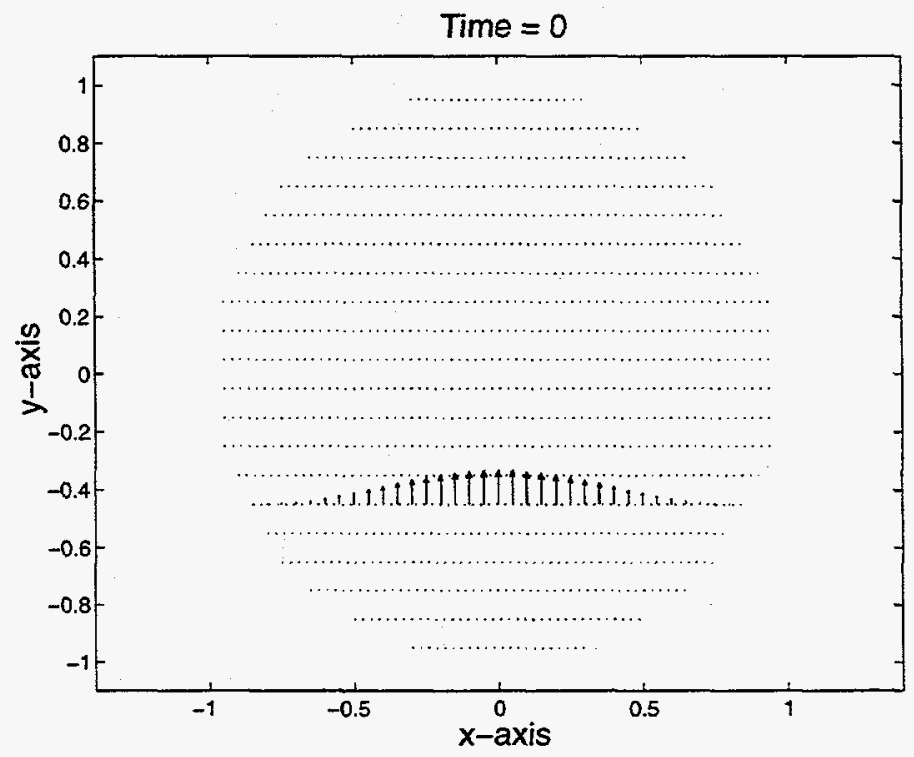

Figure 4.4: Initial m-orthogonal surfaces and the impulse defined on one of them. 


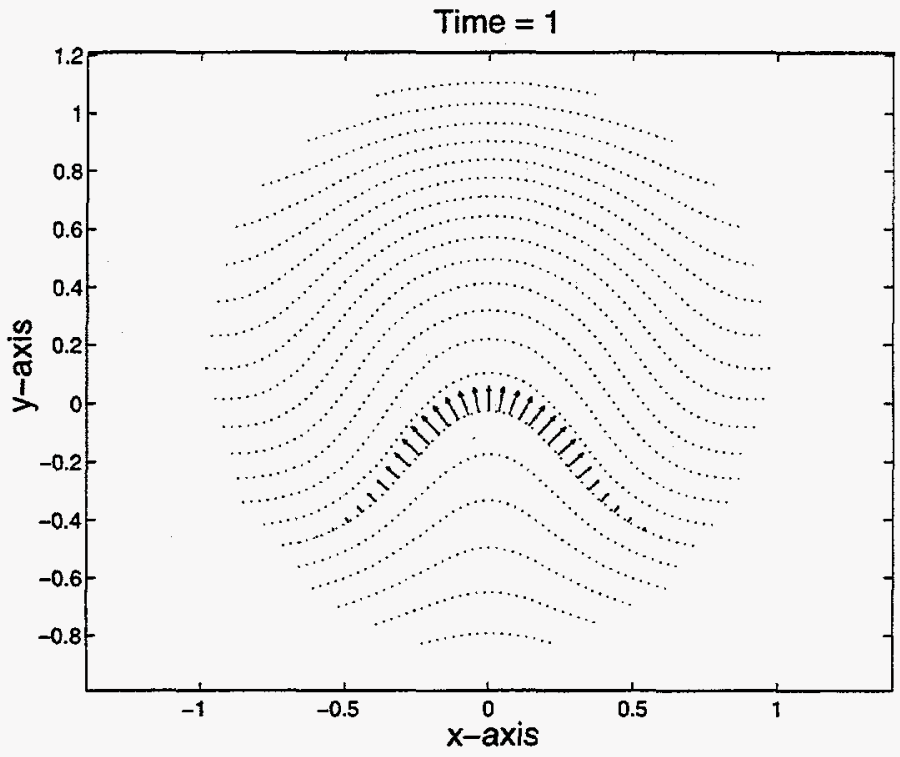

Figure 4.5: m-orthogonal surfaces and the impulse defined on one of them at time $t=1$.

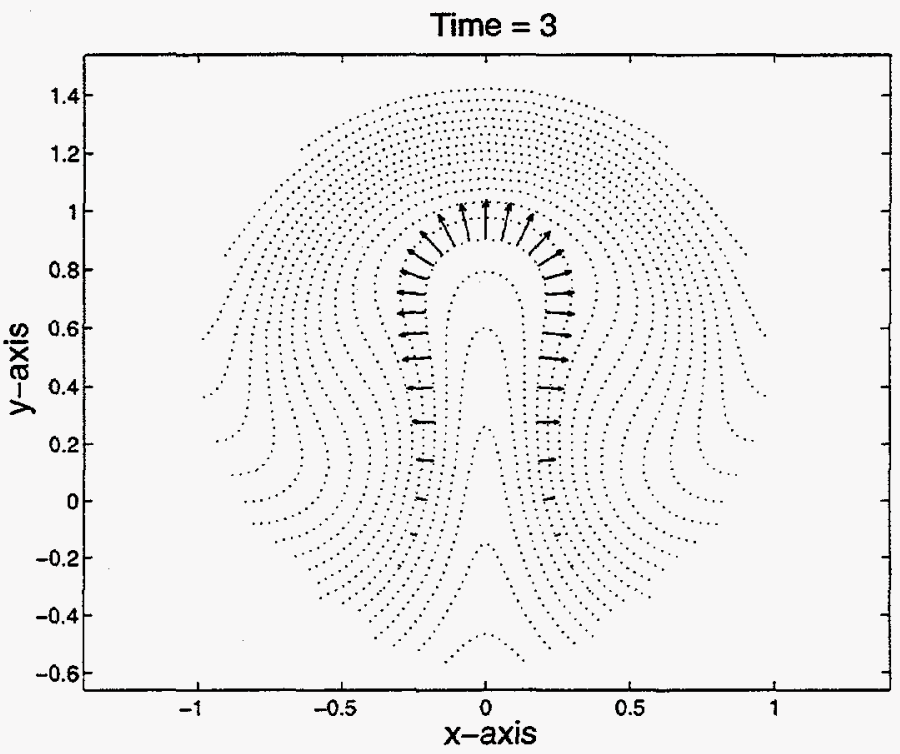

Figure 4.6: m-orthogonal surfaces and the impulse defined on one of them at time $t=3$. 
One readily notices that in regions where the surfaces come close to one another, the surfaces must stretch in order to keep the flow incompressible. As discussed in chapter 3 , the largest growth of vector magnitudes, relative to their initial values, occurs in these regions. If one interprets the impulse discretization along one surface as a quadrature approximation to a line integral on that surface, stretching of the $\mathbf{m}$-orthogonal surfaces translates into larger spacing between particles along some regions of the surface. This will lead to loss of accuracy in the line integral approximation, and numerical solutions which cannot be trusted for long times. The particles and the impulse have been updated with a fifth-order Runge-Kutta method and some discrete invariants have been tracked. The normalized kinetic energy (the Hamiltonian) was conserved to an accuracy of $1 \times 10^{-6}$, and the total impulse and angular momentum were conserved to more than 9 significant digits.

One concludes that in spite of the conservation of invariants of the Hamiltonian system of dipoles, the accuracy with which the collection of vortex dipoles represents the flow induced by the vorticity may decrease in time. As the m-orthogonal surfaces stretch, there are too few impulse vectors to provide an accurate discretization of the line integrals discussed in the previous section. At later times, the flow described by the collection of dipoles may not resemble the intended flow. Numerical experiments which address the issue of accuracy loss are discussed in the next chapter. 


\section{Chapter 5}

\section{Accuracy Considerations in Two Dimensions}

This chapter presents numerical experiments designed to illustrate how impulse discretizations which initially approximate accurately the flow induced by vortices, may evolve into configurations which no longer approximate the correct flow. This is due to a loss of accuracy in the approximation of the velocity integral discussed in the previous chapter. Two different methods for avoiding this loss of accuracy are then presented.

\subsection{Numerical Experiments}

Two numerical experiments are presented in this section. The first one shows the flow induced by two vortex blobs of opposite strength. This simple situation will be used to exemplify the way in which the impulse method can lose the accuracy with which it approximates the flow induced by the vortices. The second experiment shows two circular vortex patches of constant and opposite vorticity. The patches are discretized by vortices and this discretization is approximated by impulse vectors. This experiment was used to compute the order of accuracy of two methods for maintaining accuracy over time. These are presented in subsequent sections. 


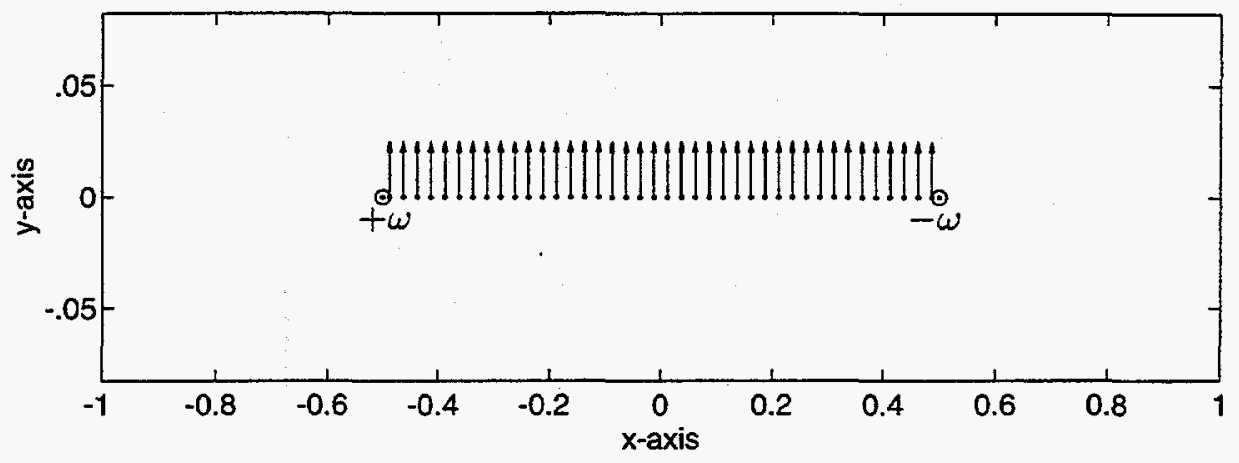

Figure 5.1: Initial impulse which represents the flow induced by two vortex blobs of equal and opposite strength.

\subsubsection{First Experiment: Two Vortex Blobs}

The first example consists of the flow induced by two point vortices of equal but opposite strength $\omega$ as shown in figure 5.1. The positive vortex is located initially at $\alpha=(-0.5,0)$ and the negative one at $\beta=(0.5,0)$. The initial impulse is $\mathbf{m}=\left(m_{1}, m_{2}\right)^{T}$ where $m_{1} \equiv 0$ and $m_{2}(x, y)=\omega$ for $-0.5<x<0.5, y=0$ and zero everywhere else.

If a vortex method is used to solve this problem, the velocity at an arbitrary point $\mathbf{x}=(x, y)$ and time $t=0$ is given by

$$
\left.\mathbf{u}(\mathbf{x})=\frac{\omega}{2 \pi}\left[(-y, x+0.5) \frac{F\left(r_{1}\right)}{r_{1}^{2}}\right]-(-y, x-0.5) \frac{F\left(r_{2}\right)}{r_{2}^{2}}\right]
$$

where $r_{1}=|\mathbf{x}-\alpha|, r_{2}=|\mathbf{x}-\beta|$, and $F$ is the shape factor. In particular, each of the point vortices moves in the $y$-direction with speed $\omega / 2 \pi$. The interval $[-1 / 2,1 / 2]$ on the $x$-axis was divided into $N$ intervals of length $h=1 / N$, and the initial impulse vectors were placed at the center of each interval. The values $N=40$ and $\omega=1$ were used. With this setup, the velocity induced by the impulse represents the midpoint rule approximation of the velocity field obtained by a vortex method which uses the same cutoff function. As the flow evolves, the integration path will wrap around the vortices and will swell up in the center. When this happens, the impulse vectors are sparsely distributed along the integration path, and one can expect the midpoint rule to experience accuracy loss. The problem was first solved with a vortex method keeping track of the location of the vortices and of marker particles placed initially where the impulse vectors start out. The same problem was then solved using the impulse method. Both methods used the 4th-order cutoff function derived from 


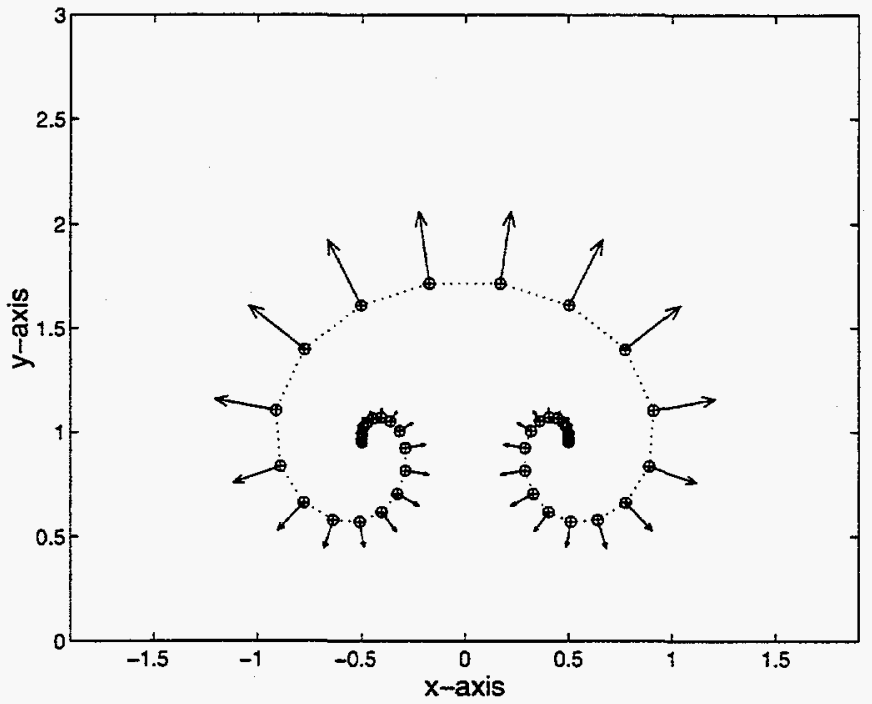

Figure 5.2: Solutions at time $t=6$ obtained with a vortex method $(\oplus)$ and the impulse method (arrows).

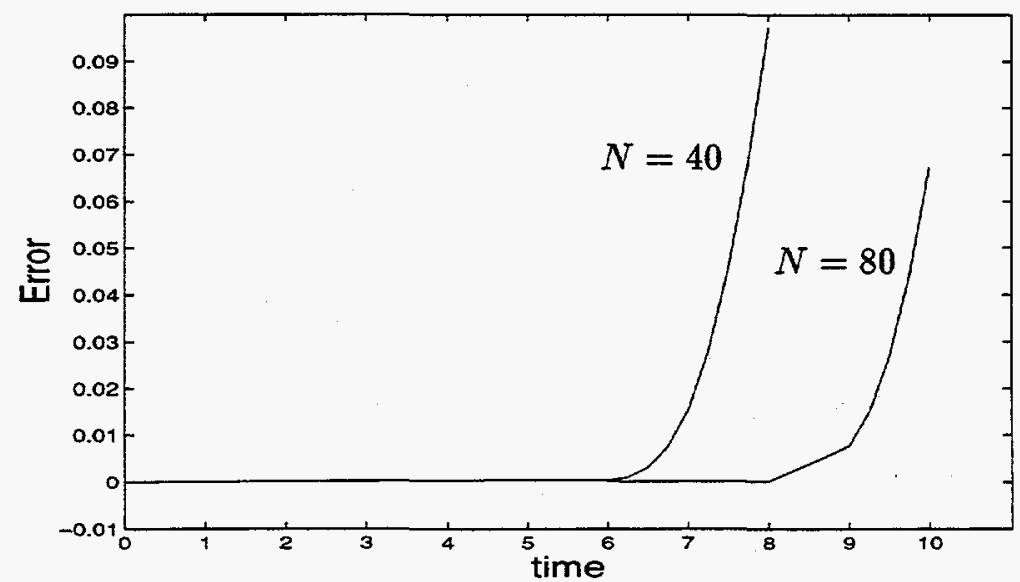

Figure 5.3: Error in particle positions for the impulse method for time $0 \leq t<10$ and discretizations corresponding to $N=40$ and $N=80$. The error was defined as the average difference in particles positions evolved once with the vortex method and once with the impulse method. 
$f_{1}(r)=\frac{1}{2 \pi}\left(e^{-r^{2}}+\frac{1}{2} e^{-r^{2} / 2}\right)$ and cutoff parameter $\delta=0.3$.

The solutions at time $t=6$ are shown in figure 5.2. The figure shows the location of the marker particles obtained with the vortex method, as well as the impulse vectors and their locations as obtained with the impulse method. The solutions do not differ appreciatively in spite of the increased separation between contiguous impulse vectors. The particles at the endpoints of the curve are the vortices. As a way to measure the discrepancy in the particle positions obtained with the impulse method in comparison with the vortex method, one can define the error

$$
\text { Error }=\frac{1}{N} \sum_{j=1}^{N}\left|\mathbf{x}_{V}^{j}-\mathbf{x}_{I}^{j}\right|
$$

where $x_{V}^{j}$ is the location of the $j$-th particle obtained with the vortex method and $x_{I}^{j}$ is the location obtained with the impulse method. Figure 5.3 shows the variation of this error in time for two different discretizations corresponding to $N=40$ and $N=80$. For times $t \leq 6$, the two solutions agree well. For later times and $N=40$, the error increases drastically and the solutions begin to differ significantly (see Figure 5.4). As expected, a finer discretization allows the impulse method to maintain good accuracy for longer times. In both cases, the sharp increase of the error occurred when the largest separation between contiguous vectors was approximately equal to the cutoff parameter $\delta$.

Figure 5.4 shows the solutions at time $t=9$ and $N=40$. Obviously, the flow induced by the impulse no longer approximates the flow induced by the two vortex blobs. The vectors are so far apart that they do not approximate the right flow. One may think of the impulse flow as that of vortex dipoles at the base of the vectors. The positions found with the vortex method indicate the locations where the impulse vectors should be. However, at this time more vectors are needed to accurately approximate the corresponding line integral along the twisted curve. Even as the impulse method loses the accuracy with which it approximates the flow induced by the two vortices, the invariants associated with the Hamiltonian system continue to be preserved to an accuracy of $10^{-8}$.

\subsubsection{Second Experiment: Two Vortex Patches}

A new problem consists of the discretization of two vortex patches of radius 0.25 , strengths +1 and -1 , and centered at $(-0.5,0)$ and $(0.5,0)$ respectively, as shown in figure 5.5. In the impulse method, vortex pairs were represented by impulse vectors pointing 


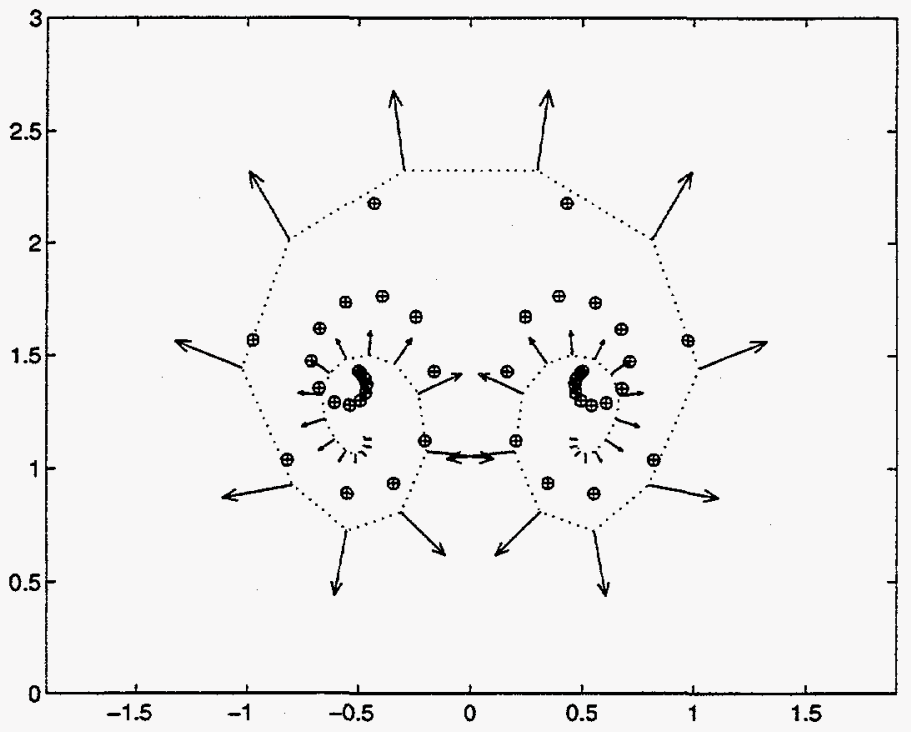

Figure 5.4: Solutions at time $t=9$ obtained with a vortex method $(\oplus)$ and the impulse method (arrows) and $N=40$.

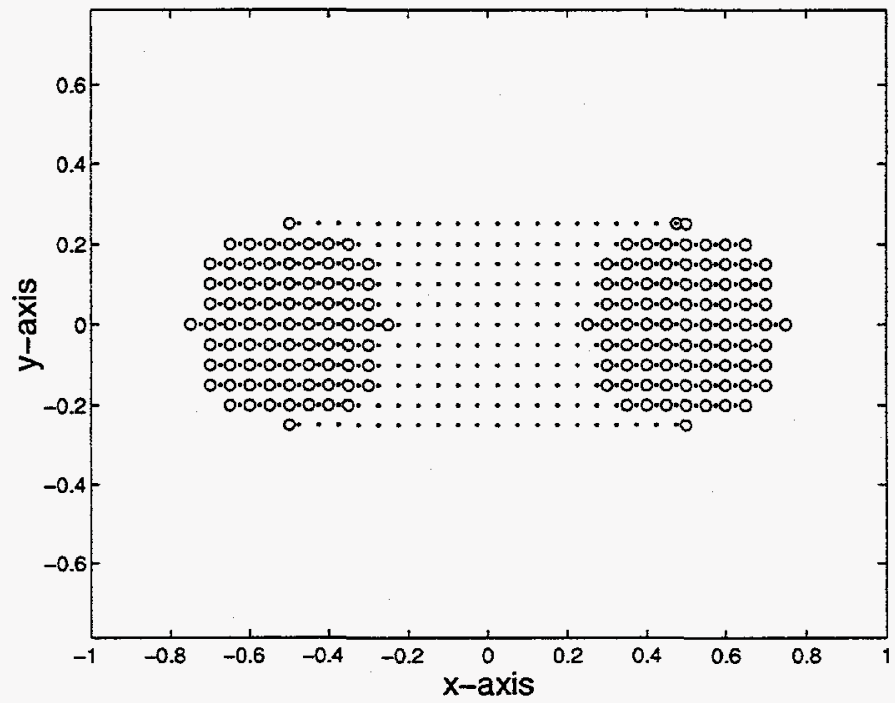

Figure 5.5: Initial discretization of the vortex patches with vortices (circles) and location of impulse vectors (dots). 
in the positive $y$-direction as in the preceding experiment, but some vortex pairs share impulse vectors with other pairs in order to keep the particle number as low as possible. The situation is the same as the one illustrated in figure 4.3. In this case, the parameters used were $\delta=0.5$ and the inter-particle spacing $h=0.05$. The particles were evolved with the
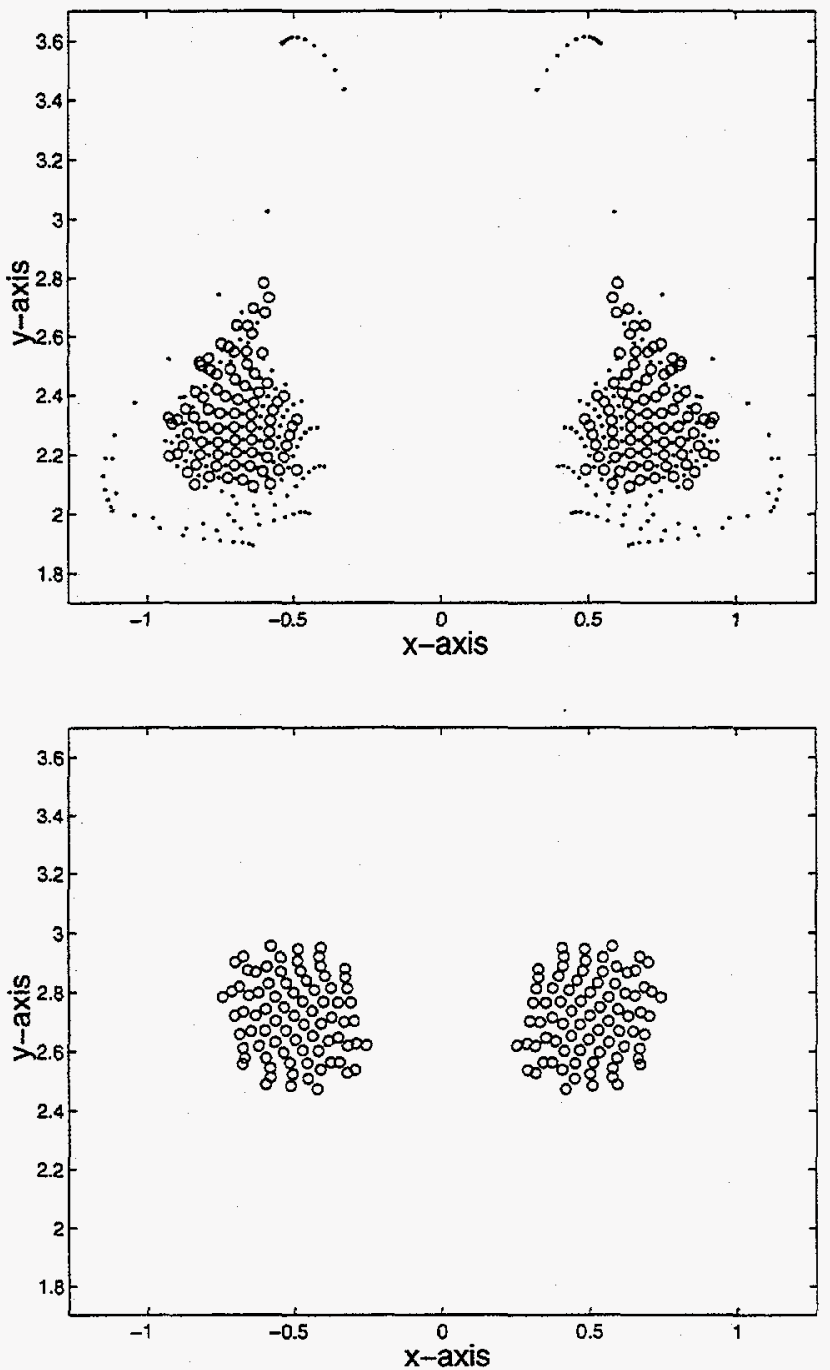

Figure 5.6: Solution at $t=4.76$. The top figure shows the vortex locations (circles) and the location of the impulse vectors (dots) obtained with the impulse method. The bottom figure shows the location of the vortices obtained with the vortex method.

impulse method to time $t=4.76$ and the positions of the vortices were compared with the ones obtained with the vortex method. Figure 5.6 shows the location of the vortices (circles) and impulse vectors (dots) given by the impulse method at $t=4.76$ and the locations of 
the vortices obtained with the vortex method. The vortex positions given by the vortex method indicate that the patches should still be approximately circular, with centers about 0.5 units higher than the locations given by the impulse method. The patches found with the impulse method are already coming apart.

\subsection{Impulse Method With Refinement}

The accuracy in the impulse approximation of the velocity field given by the vortex method can be maintained by adding new impulse vectors throughout the evolution of the flow. This technique is referred to as the impulse method with refinement. In this method, new vectors are introduced whenever the separation between contiguous vectors surpasses a predetermined length. The refinement refers to the approximation of the vortex flow by impulse vectors, not to new vortex blob discretizations of the patches. In other words, the number of vortex blobs is fixed in the beginning of the problem. The procedure to place a new vector is as follows: each of the m-orthogonal curves is piece-wise linear with a vector at the midpoint of each linear piece (see figure 5.7). If a linear piece is too long, replace the vector by two vectors in the same direction and with half the magnitude of the original one. The new vectors are equally spaced along the piece of curve. This procedure

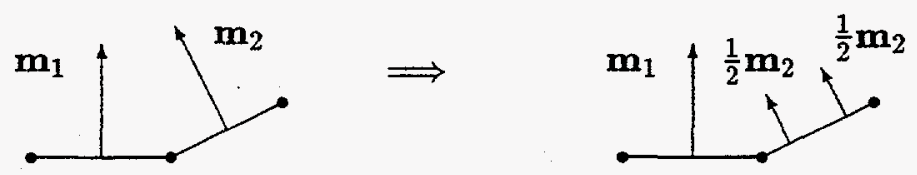

Figure 5.7: Refinement procedure which preserves impulse. The vector $\mathbf{m}_{2}$ is replaced by two vectors in the same direction and of half the magnitude of the original.

is designed to preserve the discrete impulse, $\mathbf{I}=\sum \mathbf{m}^{j}$, exactly. Invariants which depend on the discretization, such as the energy, cannot be expected to be preserved as well as impulse since the discretization is being changed. Even without the dynamics, changes in the number of vectors of a discretization, say of figure 5.1, lead to slight changes in the total energy due to its sensitivity to the discretization. However, one can expect to conserve all invariants approximately during the refinement procedure.

The results of the impulse method with refinement for the problem of two vortex 


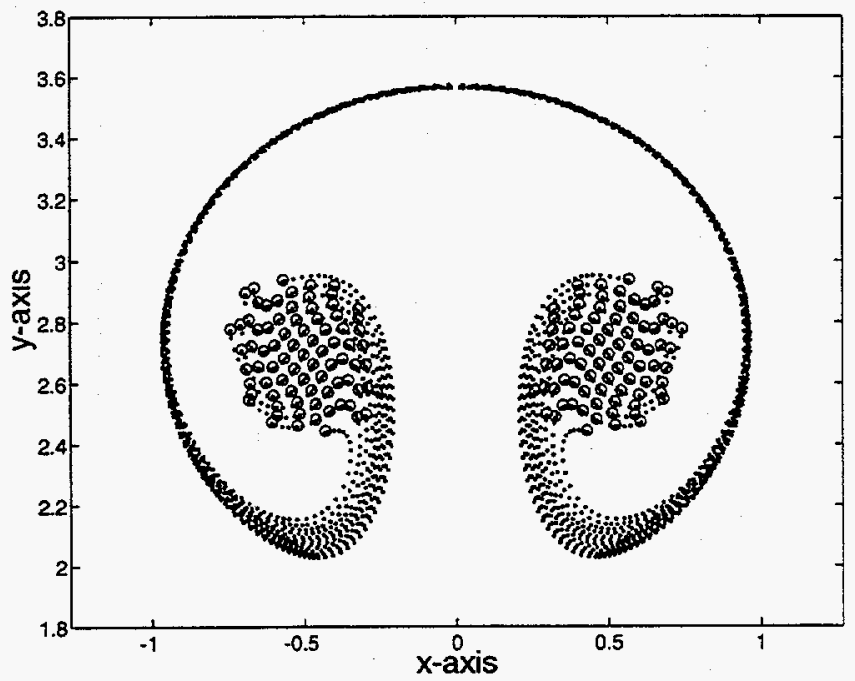

Figure 5.8: Solution at $t=4.72$ found with the impulse method with refinement.

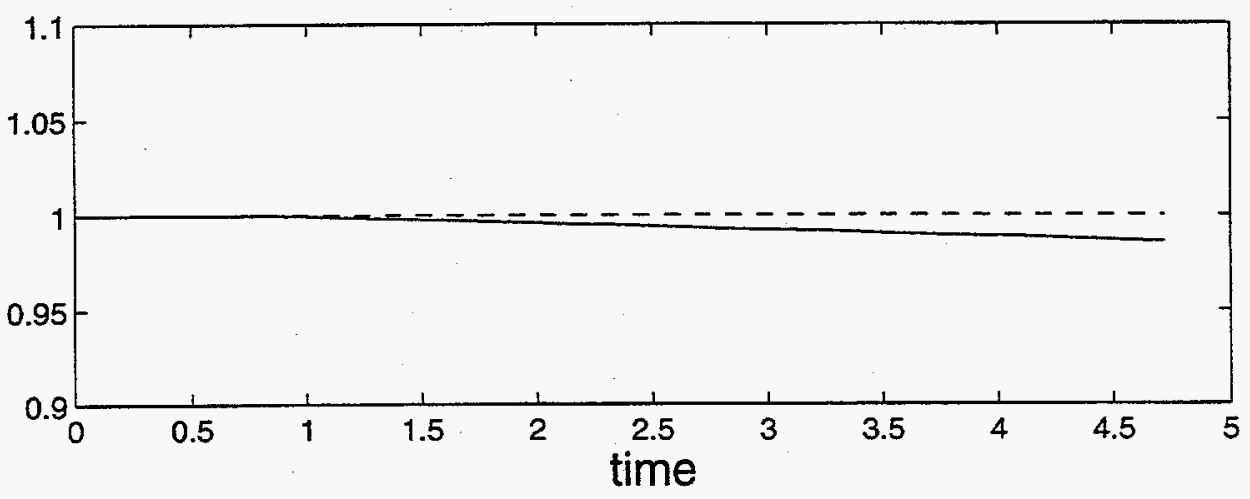

Figure 5.9: Normalized impulse (dashed) and energy (solid) at $t=4.72$ found with the impulse method with refinement. 
patches are shown in figure 5.8 at time $t=4.72$. In this case, an impulse vector was replaced by two vectors whenever the discretization size (the inter-particle spacing) reached twice the value of the original discretization, which was set to $h=0.05$. In comparison with figure 5.6, it is clear that the refinement procedure allows the impulse method to maintain the accuracy with which it approximates the flow. The approximate conservation of energy and exact conservation of impulse are shown in figure 5.9. However, the computation using refinement becomes increasingly expensive in time, since the number of impulse variables continually increases. For instance, the number of particles used to represent the initial flow was 290, while in figure 5.6 (at time 4.72) there are 1180 particles. When the velocity at each particle is computed directly, i.e. when the contribution to the velocity at each particle location due to every particle is computed separately, then the number of function evaluations for $N$ particles is of $O\left(N^{2}\right)$. Without the use of fast summation techniques, the impulse method with refinement eventually becomes prohibitively expensive.

In order to estimate the order of accuracy of the impulse approximation to the flow induced by the vortices, the same vortex discretization was approximated by impulse vectors with initial discretization $h=0.025$. The solution was carried to $t=4.72$ and compared with the vortex method solution. This final time was chosen arbitrarily as representative of times when the original impulse method is known to yield wrong answers. At other final times, the results were similar. As shown in figure 5.10, the discrepancies in the vortex positions decreased by about a factor of 4 . This indicates that the impulse method with refinement is second order accurate. The previous chapter explained that the impulse flow was the midpoint-rule approximation of the vortex flow, and thus should be second order accurate in space. The results support this statement.

\subsubsection{Return to the Example in $\$ 3.7$}

An example of a large smooth vortex dipole taken from [7] was presented at the end of the last chapter (refer to figures 4.4-4.6). The results there and in [7] were shown at times too short for the accuracy loss to be perceived visually. Figure 5.11 shows the solution of the same problem at a later time. In this figure it is evident that the flow represented by the impulse is different from the original flow. Material curves are no longer resolved between contiguous vectors as these curves appear to cross one another. Figure 5.12 shows the solution at the same time obtained with the impulse method with refinement. 

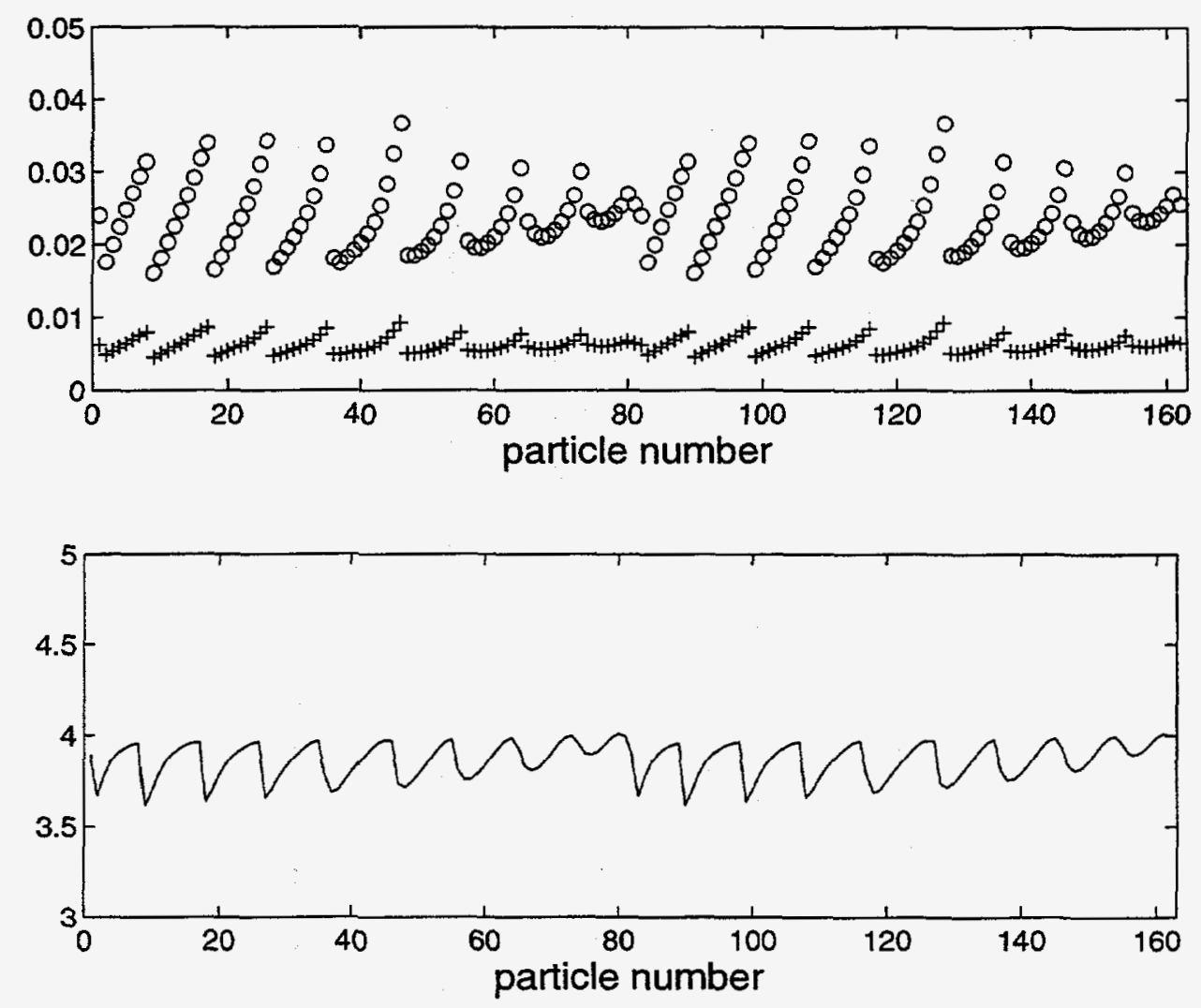

Figure 5.10: Comparison of vortex positions at $t=4.72$. The top figure shows the distance between corresponding vortices given by the impulse method with refinement for $h=0.05$ (circles) and $h=0.025$ (crosses), compared with the vortex method. The bottom figure shows the ratio of the distances. 


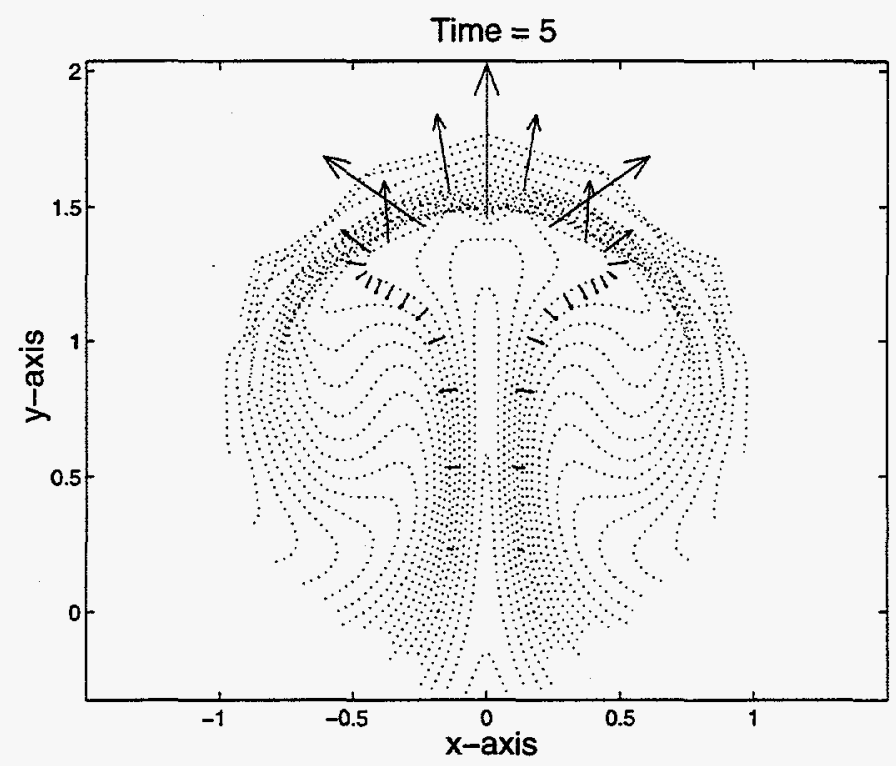

Figure 5.11: m-orthogonal surfaces and the impulse defined on one of them at time $t=5$. Solution obtained with the impulse method.

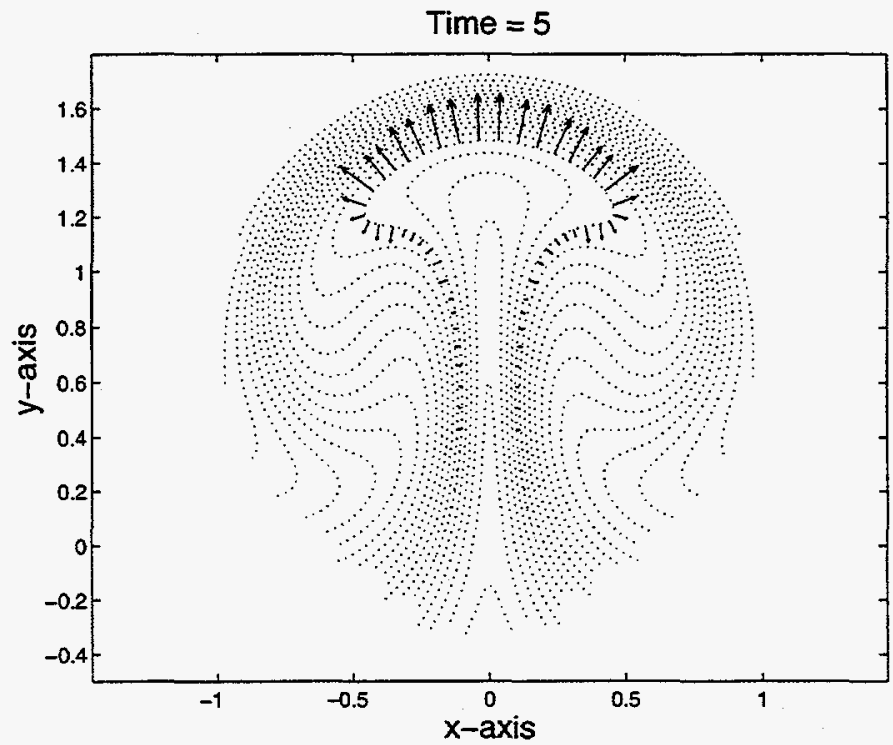

Figure 5.12: m-orthogonal surfaces and the impulse defined on one of them at time $t=5$. Solution obtained with the impulse method with refinement. 
The refinement parameter used was $2 h$, where $h$ was the original inter-particle spacing. The material curves remain smooth and do not cross one another. Figure 5.13 shows the increase in the number of particles for this problem using the impulse method with refinement.

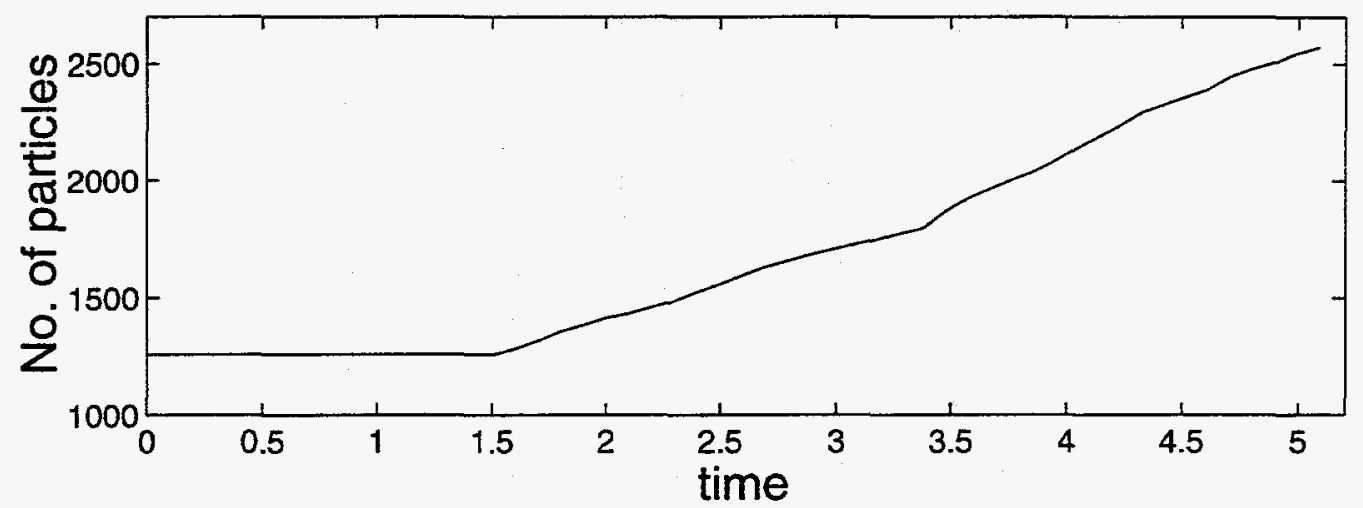

Figure 5.13: Variation in the number of particles used in the impulse method with refinement for the problem of $\S 3.7$.

\subsection{Impulse Method With Resurfacing}

One would like to have a method that maintains the initial accuracy of the impulse discretization and in which the number of particles used does not grow without bound. A second variant of the impulse method, impulse method with resurfacing, is introduced now. In figure 5.2, one can stop the evolution of the flow and replace the impulse field (and the curve along which it is defined) by a new field along a straight line connecting the vortices, which are located at the endpoints of the curve. The non-uniqueness of the impulse field that represents a given flow is what allows this replacement. In the case of the two vortex blobs of the first example in this chapter, any curve that connects the two vortices can be used to define an impulse field. This field would be perpendicular to the curve and of constant magnitude along it.

In the impulse method with resurfacing, the impulse vectors along a curve connecting a vortex pair are evolved a few time steps and then the vectors are discarded and a new curve is placed connecting the pair. For curves that connect several vortex pairs, as in the example with the patches, the new curves are chosen to be shorter and nearly horizontal. This prevents the total number of impulse vectors needed to represent the flow 
from growing unboundedly and allows the computation of solutions for longer times.

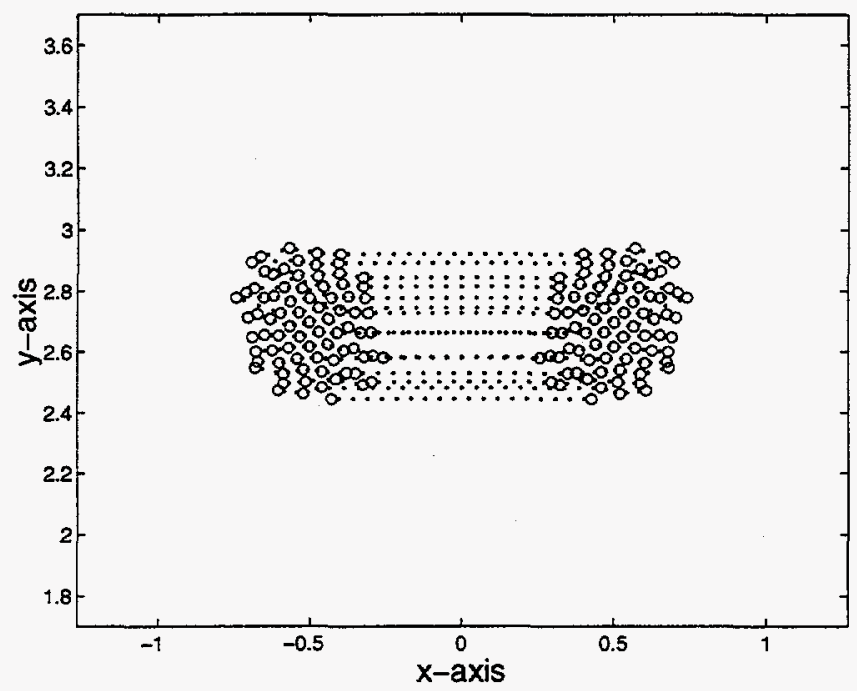

Figure 5.14: Solution at $t=4.72$ found with the impulse method with resurfacing with $\delta=0.5$ and $h=0.05$.

The problem of the vortex patches was solved using the impulse method with resurfacing. Figure 5.14 shows the particle positions obtained with this method at time $t=4.72$ as in the previous figures. In this case, the new $\mathbf{m}$-orthogonal curves are replaced at the end of every time step to ensure that the initial discretization defines the accuracy of the method. One can define the error in the position of the vortices as in Eq. (5.1)

$$
\text { Error }=\frac{1}{N} \sum_{j=1}^{N}\left|\mathbf{x}_{V}^{j}-\mathbf{x}_{I}^{j}\right|
$$

where $\mathbf{x}_{I}^{j}$ represents the position of the $j$-th vortex obtained by the impulse method with resurfacing and $x_{V}^{j}$ represents the position of the same vortex obtained with the vortex method. Table 5.1 shows the results from two impulse discretizations corresponding to inter-particle separations of $h=0.05$ and $h=0.025$. The numbers in the table indicate that the errors increase linearly in time and decrease by about a factor of four when the discretization is halved. This is the same result shown in figure 5.10 for the case of the impulse method with refinement. However, the number of particles using the resurfacing procedure does not increase without bound as it does in the refinement. For instance, the number of particles used by the resurfacing procedure for the case of the vortex patches and $h=0.025$ is shown in figure 5.15. The alignment of the particles in the initial conditions 


\begin{tabular}{|c|c|c|c|c|}
\hline & \multicolumn{2}{|c|}{$h=0.05$} & \multicolumn{2}{c|}{$h=0.025$} \\
\hline time & $\max _{j}\left|\mathbf{x}_{V}^{j}-\mathbf{x}_{I}^{j}\right|$ & $\frac{1}{N} \sum_{1}^{N}\left|\mathbf{x}_{V}^{j}-\mathbf{x}_{I}^{j}\right|$ & $\max _{j}\left|\mathbf{x}_{V}^{j}-\mathbf{x}_{I}^{j}\right|$ & $\frac{1}{N} \sum_{I}^{N}\left|\mathbf{x}_{V}^{j}-\mathbf{x}_{I}^{j}\right|$ \\
\hline \hline$t=2.4$ & $7.4646 \mathrm{e}-04$ & $4.2806 \mathrm{e}-04$ & $1.8560 \mathrm{e}-04$ & $1.1063 \mathrm{e}-04$ \\
\hline$t=4.72$ & $13.431 \mathrm{e}-04$ & $8.0132 \mathrm{e}-04$ & $3.5754 \mathrm{e}-04$ & $2.0817 \mathrm{e}-04$ \\
\hline$t=7.92$ & $22.859 \mathrm{e}-04$ & $12.648 \mathrm{e}-04$ & $6.1270 \mathrm{e}-04$ & $3.3106 \mathrm{e}-04$ \\
\hline
\end{tabular}

Table 5.1: Errors for the vortex patch problem using the impulse method with resurfacing and two different discretizations. The method used cutoff parameter $\delta=0.5$ and time step $\Delta t=0.08 . \mathbf{x}_{V}^{j}$ is the position of the $j$-th particle obtained with the vortex method, $\mathbf{x}_{I}^{j}$ is the position of the same particle obtained with the impulse method.

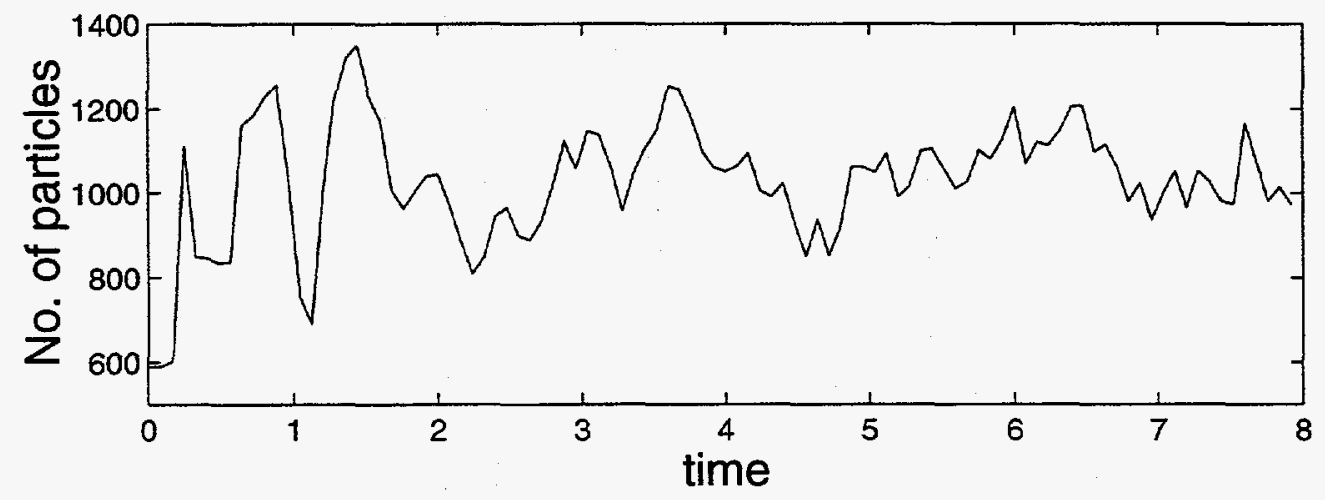

Figure 5.15: Variation in the number of particles used in the impulse method with resurfacing for $h=0.025, \delta=0.5$ and $\Delta t=0.08$. 
allows the number of particles to be the smallest at time $t=0$. In this example, the initial number of particles was 590. The number of particles at later times fluctuates about 1000 .

The errors were also computed for three separate runs of the impulse method with refinement performing the resurfacing every 1,5 and 10 time steps respectively. These errors are shown in table 5.2 and correspond to $\frac{1}{N} \sum_{1}^{N}\left|\mathbf{x}_{V}^{j}-\mathbf{x}_{I}^{j}\right|$. The errors increase as the frequency with which the resurfacing is performed decreases. This is to be expected since the separation between particles along an $\mathbf{m}$-orthogonal curve is larger after 10 steps than the separation after only one step. Table 5.3 shows the variation of the errors for different time steps.

\begin{tabular}{|c|c|c|c|}
\hline Time & $\begin{array}{l}\text { Resurfacing every } \\
\text { iteration }\end{array}$ & $\begin{array}{l}\text { Resurfacing every } \\
\text { 5 iterations }\end{array}$ & $\begin{array}{l}\text { Resurfacing every } \\
\text { 10 iterations }\end{array}$ \\
\hline \hline$t=2.4$ & $4.2806 \mathrm{e}-04$ & $4.8407 \mathrm{e}-04$ & $6.7586 \mathrm{e}-04$ \\
\hline$t=4.72$ & $8.0132 \mathrm{e}-04$ & $9.4746 \mathrm{e}-04$ & $15.485 \mathrm{e}-04$ \\
\hline$t=7.92$ & $12.648 \mathrm{e}-04$ & $21.101 \mathrm{e}-04$ & $36.562 \mathrm{e}-04$ \\
\hline
\end{tabular}

Table 5.2: Errors for the impulse method with resurfacing using three frequencies of resurfacing. The method used cutoff parameter $\delta=0.5, h=0.05$ and time step $\Delta t=0.08$.

\begin{tabular}{|c|c|c|c|c|}
\hline & \multicolumn{2}{|c|}{$\Delta t=0.125$} & \multicolumn{2}{c|}{$\Delta t=0.25$} \\
\hline time & $\max _{j}\left|\mathrm{x}_{V}^{j}-\mathrm{x}_{I}^{j}\right|$ & $\frac{1}{N} \sum_{1}^{N}\left|\mathrm{x}_{V}^{j}-\mathrm{x}_{I}^{j}\right|$ & $\max _{j}\left|\mathrm{x}_{V}^{j}-\mathrm{x}_{I}^{j}\right|$ & $\frac{1}{N} \sum_{I}^{N}\left|\mathrm{x}_{V}^{j}-\mathrm{x}_{I}^{j}\right|$ \\
\hline \hline$t=1.25$ & $2.1677 \mathrm{e}-03$ & $1.1738 \mathrm{e}-03$ & $3.1036 \mathrm{e}-03$ & $1.9639 \mathrm{e}-03$ \\
\hline$t=2.50$ & $3.6319 \mathrm{e}-03$ & $2.2104 \mathrm{e}-03$ & $4.9524 \mathrm{e}-03$ & $2.6307 \mathrm{e}-03$ \\
\hline$t=3.75$ & $4.6283 \mathrm{e}-03$ & $3.2911 \mathrm{e}-03$ & $9.4152 \mathrm{e}-03$ & $3.8728 \mathrm{e}-03$ \\
\hline
\end{tabular}

Table 5.3: Errors for the vortex patch problem using the impulse method with resurfacing and two different time steps. The method used $\delta=0.25$ and discretization size $h=0.05$. 


\subsubsection{Finding Vortex Positions From the Impulse Discretization}

The impulse method with resurfacing discussed in the previous section treated the vortices as marker particles and used their positions to define the new surfaces and to compare the results with those obtained with the vortex method. In general, one must be able to extract information about the vorticity in the flow from the impulse discretization, without having to track the vortices in addition to the impulse variables. This section explains a procedure for locating vortices which induce a flow equal to the flow induced by the impulse vectors up to $O\left(h_{0}^{2}\right)$, for a prescribed parameter $h_{0}$.

Consider the velocity field due to an impulse vector $\mathbf{m}$ located at the origin and pointing in the positive $y$-direction. This velocity field is $\mathbf{u}(\mathbf{x})=|\mathbf{m}| \partial_{x} \mathbf{K}_{\delta}(\mathbf{x})$, where $\mathbf{K}_{\delta}=\mathbf{K} * f_{\delta}$ is the smoothed vortex kernel from $\S 4.6$. Assume that $h_{0}$ is given and that $\mathbf{K}_{\delta}$ has at least three continuous derivatives. Then the velocity above can be written as

$$
\begin{aligned}
\mathbf{u}(\mathbf{x}) & =|\mathbf{m}|\left\{\frac{1}{2 h_{0}}\left[\mathbf{K}_{\delta}\left(x+h_{0}, y\right)-\mathbf{K}_{\delta}\left(x-h_{0}, y\right)\right]+\frac{h_{0}^{2}}{6} \partial_{x}^{3} \mathbf{K}_{\delta}(\zeta, y)\right\} \\
& =\xi\left[\mathbf{K}_{\delta}\left(x+h_{0}, y\right)-\mathbf{K}_{\delta}\left(x-h_{0}, y\right)\right]-\frac{\xi h_{0}^{3}}{3} \partial_{x}^{3} \mathbf{K}_{\delta}(\zeta, y),
\end{aligned}
$$

where $\xi=|\mathbf{m}| / 2 h_{0}$ and $\zeta$ is some number between $-h_{0}$ and $h_{0}$.

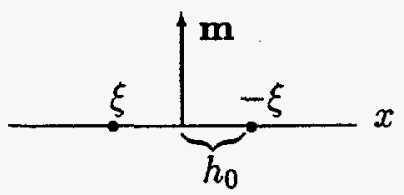

An error of size up to $\frac{1}{3} \xi h_{0}^{3}\left\|\partial_{x}^{3} \mathbf{K}_{\delta}\right\|$ has been committed in the replacement of an impulse vector by two vortices at a distance $h_{0}$ on either side of the vector. This is the impulse discretization error. Vortices close to the vector induce a better approximation to the impulse flow than weaker vortices placed farther apart. If two or more vortices created in this way are located at the same point, they can be combined into one vortex with strength equal to the sum of the strengths of all the vortices that have been combined. No vortex is needed if the combined vorticity is zero. In practice, two vortices will not generally be placed at the exact position. However, there is some flexibility in the placement of the vortices which can be deduced from the following error analysis. Let $\mathbf{a}=\left(a_{1}, a_{2}\right)^{T}$ and suppose one changes the position of the left vortex to the point $-\left(h_{0}+a_{1}, a_{2}\right)$ instead of 
$-\left(h_{0}, 0\right)$. Then a Taylor series expansion of $\mathbf{K}_{\delta}(x, y)$ yields

$$
\mathbf{K}_{\delta}\left(x+h_{0}+a_{1}, y+a_{2}\right)=\mathbf{K}_{\delta}\left(x+h_{0}, y\right)+\mathbf{a} \cdot D \mathbf{K}_{\delta}\left(\eta_{1}, \eta_{2}\right),
$$

where $\left(\eta_{1}, \eta_{2}\right)$ is a point in a ball of radius $|\mathbf{a}|$ centered at $\left(-h_{0}, 0\right)$. A balance of the error due to the change in location of one vortex and the impulse discretization error leads to $|\mathbf{a}| \leq \frac{1}{3} h_{0}^{3}\left\|\partial_{x}^{3} \mathbf{K}_{\delta}\right\| /\left\|D \mathbf{K}_{\delta}\right\|$. The same could be said about the vortex on the right.

The norms above depend only on the cutoff function and can be computed in advance once the choice of cutoff function has been made. From the definition of the shape factor $F(r)$, it is clear that we can write $F(r)=q^{2} G(q)$, where $q=r / \delta$ and $G(q) \neq$ 0 anywhere. Therefore, the vortex kernel can be written in polar coordinates as $K_{\delta}=$ $\hat{\theta} q G(q) \delta^{-1}$. Upon differentiation of this expression with respect to $x$ or $y$ we conclude that $D \mathbf{K}_{\delta}(x, y)$ scales as $\delta^{-2}$ and $\partial_{x}^{3} \mathbf{K}_{\delta}(x, y)$ scales as $\delta^{-4}$. Thus for the error terms to be of the same order, the vector a must satisfy

$$
|\mathbf{a}|=\frac{h_{0}^{3}}{3}\left\|\partial_{x}^{3} \mathbf{K}_{\delta}\right\| /\left\|D \mathbf{K}_{\delta}\right\|=O\left(h_{0}^{3} \delta^{-2}\right)
$$

so vortices can be placed within $O\left(h_{0}^{3} \delta^{-2}\right)$ of $\pm\left(h_{0}, 0\right)$ without incurring larger errors than the discretization errors. This scaling is independent of the cutoff function used; the dimensionless expressions $\delta^{2} D \mathbf{K}_{\delta}$ and $\delta^{4} \partial_{x}^{3} \mathbf{K}_{\delta}$ depend on the cutoff function but can be computed once and for all for each function.

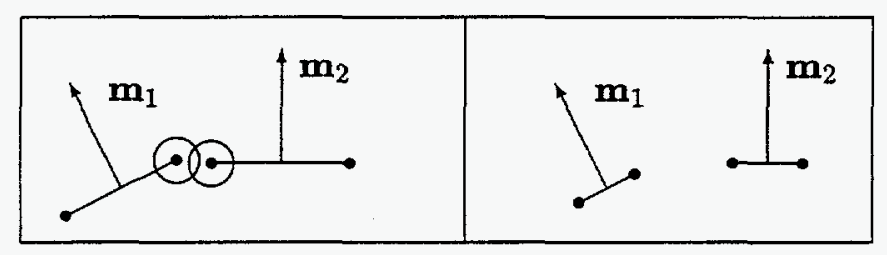

Figure 5.16: The vectors $\mathbf{m}_{1}$ and $\mathbf{m}_{2}$ can be replaced by two pairs of vortices located near the base of the vectors. The left picture shows a low accuracy representation of the impulse flow but allows the combination of the middle vortices. The right picture shows a more accurate representation of the impulse flow by the vortices.

The error in the replacement of an impulse vector by two vortices is $O\left(\xi h_{0}^{3} / \delta^{4}\right)$ as indicated in Eq. (5.2). After integrating along a strip to which the vectors are perpendicular, the error becomes $O\left(\xi h_{0}^{2} / \delta^{4}\right)$. When $h_{0} / \delta=O(1)$, the discretization errors become extremely large as seen in figure 5.3 . 
The freedom in the placement of the vortices that approximate the flow induced by impulse can be put to good use in the impulse method with resurfacing. For a given collection of impulse vectors and a parameter $h_{0}$, one can find vortex pairs that approximate the flow induced by the impulse and systematically combine vortices that are within $O\left(h_{0}^{3} \delta^{-2}\right)$ of one another. The circles of the left side of figure 5.16 represent the regions where the corresponding vortices may be placed. Those vortices would be combined into a single vortex, with strength equal to the sum of the strengths of the two, placed in the middle of the intersection of the circles. This process allows the cancellation of opposite vortices within $O\left(h_{0}^{3} \delta^{-2}\right)$ of each other.

\subsubsection{Example}

Returning to the example of the two vortex patches, suppose that the impulse discretization uses initially the inter-particle spacing $h=0.05$ and cutoff radius $\delta=0.5$. The location of the impulse vectors after a short time are shown in figure 5.17. The figure

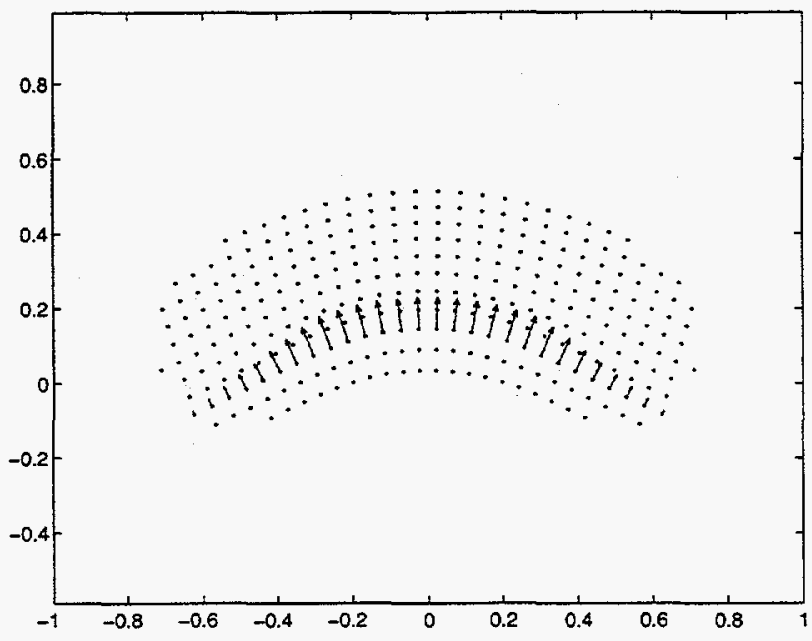

Figure 5.17: Location of impulse vectors before finding vortices. One $\mathbf{m}$-orthogonal curve shown.

also shows the impulse vectors along one m-orthogonal curve. In order to find a collection of vortices which induce the same flow as this impulse field, the impulse vectors can be replaced by vortex pairs near the base of the vectors. The strengths of the vortices are determined by the equation $2 h_{0} \omega^{j}=\left|\mathbf{m}^{j}\right|$, where the parameter $h_{0}$ is half the distance separating the vortices of a pair. At time $t=0$, the choice $2 h_{0}=h$ would lead to a discretization of the 
vortex patches by vortices separated a distance $h$. At the instance shown in figure 5.17, the impulse vectors have separated beyond the initial inter-particle spacing $h$ so that they represent the vortex patches to a lower accuracy. The vortex representation of the impulse

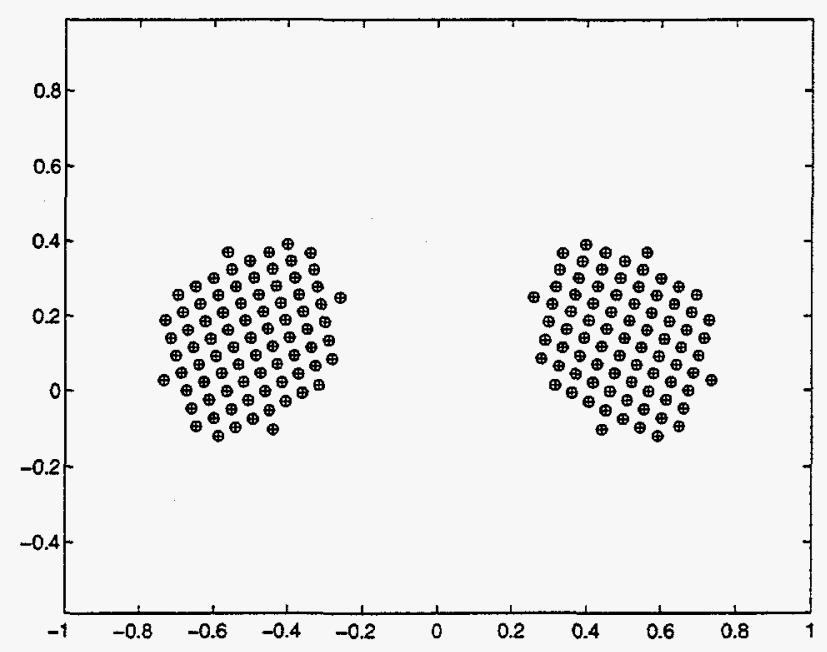

Figure 5.18: Location of vortices with $2 h_{0}=0.065$. All impulse vectors allowed the cancellation of vorticity except in the two circular patches.

flow for $2 h_{0}=1.3 h$ is shown in figure 5.18. This value of $h_{0}$ is large enough to ensure the cancellation of vorticity between the patches. If the value of $h_{0}$ is decreased, and thus a more accurate vortex representation of the impulse flow chosen, one can expect some vorticity to be left between the patches. Figure 5.19 shows the case of $2 h_{0}=1.2 h$, in which vortices from contiguous impulse vectors were combined but their strengths did not cancel due to the more severe restriction in the placement of the vortex pairs. The result is a few additional vortices whose strengths have absolute value less than 0.0045 , while the vortices in the patches have strengths \pm 1 . The difference between the flow induced by this collection of vortices and the patches is entirely due to the effect of the weak vortices. This effect is small since these vortices are far from the patches.

If one decreases the value of $h_{0}$ even more, the vortex representation of the impulse How departs further from a discretization of the two patches. Figure 5.20 shows results for the case $2 h_{0}=1.1 h$. Some vortices end up very close to vortices from adjacent impulse vectors but not close enough for them to be combined. This leaves a cloud of strong vortices between the patches. Vortices of opposite strength between the patches are so close to each other that they cannot be distinguished easily in the figure. The net effect of these vortices 


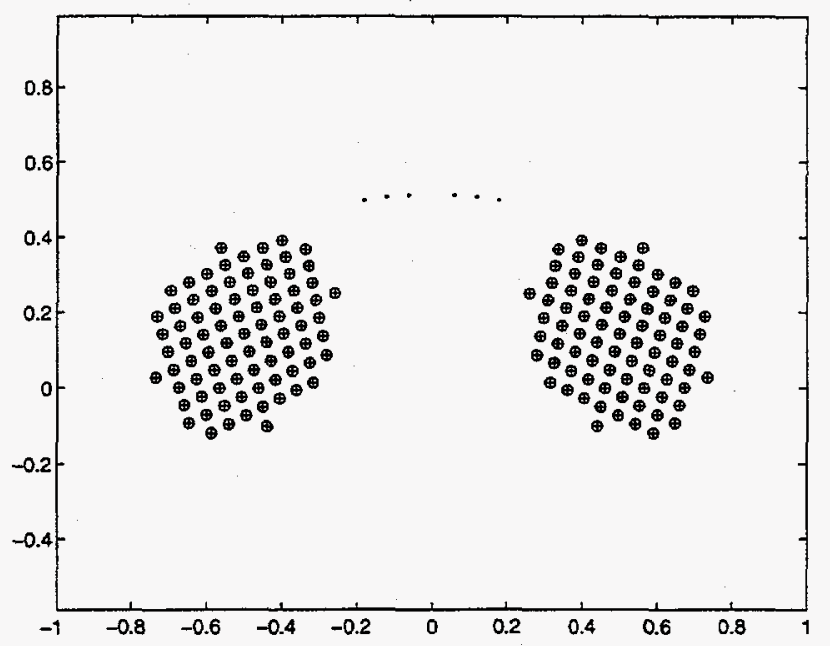

Figure 5.19: Location of vortices with $2 h_{0}=0.06$. A few vortices at the top combined but their vorticity did not cancel leaving vortices of strength smaller than 0.0045 .

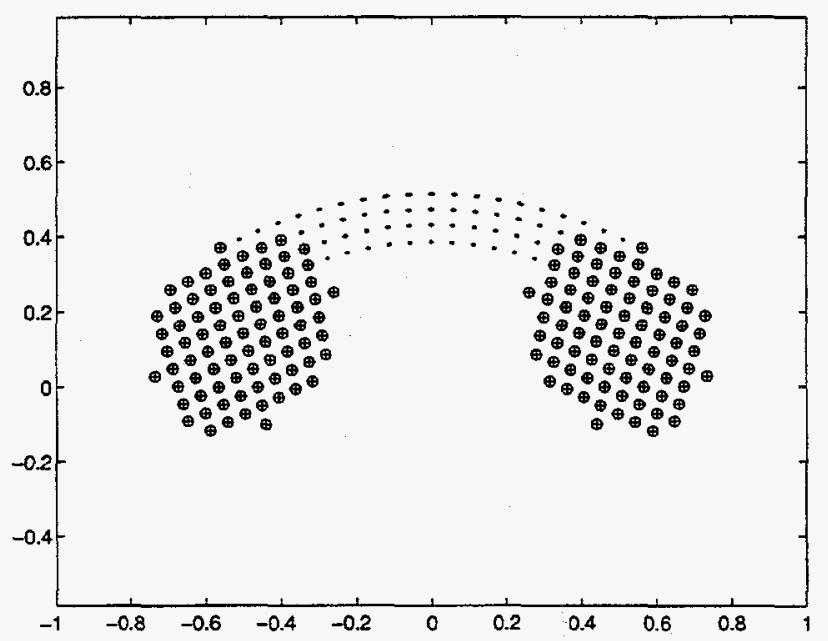

Figure 5.20: Location of vortices with $2 h_{0}=0.055$. Some impulse vectors are not close enough to their neighbors for vortices to combine. The result is pairs of vortices of strength up to \pm 10 inducing nearly opposing flows. 
is small because they induce nearly opposite flows since they are so close to each other, not because they are weak.

The last two figures show that as one finds collections of vortices that approximate the vorticity induced by a given impulse discretization to increasingly higher accuracy, the difference between the vortices found and the discretization of the patches becomes larger. This is the case since the impulse vectors represent the vortex patches to an accuracy which decreases as time increases. The small effects manifest themselves as weak vortices or as vortices (not necessarily weak) of opposite strength and very near each other inducing nearly opposite flows. In either case, the additional vortices must must be included as part of the approximation to the impulse flow. 


\section{Chapter 6}

\section{Impulse Variables in Three Dimensions}

The dipole character of the flow induced by impulse in either two or three dimensions was displayed in chapter 4 . In three dimensions, the flow induced by impulse vectors can be viewed as an approximation to the flow induced by small vortex loops. This view is more convenient for gaining insight about the role of discrete impulse in the numerical method presented in chapter 4 . This chapter begins by explaining the connection between impulse and vortex loops and then presents the three-dimensional versions of the refinement and resurfacing procedures introduced in the previous chapter. Numerical examples are presented throughout this chapter.

\subsection{Impulse and Vortex Loops}

This section presents a part of the discussion in [22] which leads to the conclusion that at sufficiently long distances, any localized vorticity distribution induces the same flow as a vortex dipole. The presentation in [22] is in the context of magnetostatics but its interpretation in fluid dynamics is clear if one keeps in mind the following correspondence between variables in the two fields:

\begin{tabular}{|l|l|}
\hline Magnetostatics & Fluid Dynamics \\
\hline current density, J & vorticity, $\xi$ \\
magnetization, $\mathbf{m}$ & impulse, $\mathbf{m}$ \\
magnetic induction, B & velocity, $\mathbf{u}$ \\
\hline
\end{tabular}


The flow induced by a localized vorticity field in the shape of a small loop can be identified with the flow induced by an impulse vector normal to the plane of the loop in the following way. Assume $\xi(\mathbf{x})$ is a vorticity distribution confined to a small set containing the origin. The flow induced by $\xi$ can be written as

$$
\mathbf{u}(\mathbf{x})=\nabla \times(G * \xi)
$$

where $G(\mathbf{x})=(4 \pi|\mathbf{x}|)^{-1}$ is the Green's function for the Laplacian operator in free space. The size of the set is assumed to be small relative to the distance from the observer; that is, $|\mathbf{y}| \ll|\mathbf{x}|$ whenever $\xi(\mathbf{y}) \neq 0$. One can make use of the expansion

$$
\frac{1}{|\mathbf{x}-\mathbf{y}|}=\frac{1}{|\mathbf{x}|}+\frac{\mathbf{x} \cdot \mathbf{y}}{|\mathbf{x}|^{3}}+\cdots
$$

to write the convolution as

$$
\begin{aligned}
(G * \xi)(\mathbf{x}) & =\frac{1}{4 \pi} \int \frac{\xi(\mathbf{y})}{|\mathbf{x}-\mathbf{y}|} d \mathbf{y} \\
& =\frac{1}{4 \pi|\mathbf{x}|} \int \xi(\mathbf{y}) d \mathbf{y}+\frac{1}{4 \pi|\mathbf{x}|^{3}} \int(\mathbf{x} \cdot \mathbf{y}) \xi(\mathbf{y}) d \mathbf{y}+\cdots
\end{aligned}
$$

where the neglected terms are no larger than $O\left(|\mathbf{y}|^{2} /|\mathbf{x}|^{3}\right)$. Since $\xi$ is divergence-free and compactly supported, it can easily be shown that the first integral in Eq. (6.1) is zero and the second integral satisfies

$$
\int(\mathbf{x} \cdot \mathbf{y}) \xi(\mathbf{y}) d \mathbf{y}=-\frac{1}{2} \mathbf{x} \times \int \mathbf{y} \times \xi(\mathbf{y}) d \mathbf{y} .
$$

Then the first non-zero term in the Taylor expansion of the Green's function yields

$$
(G * \xi)(\mathbf{x})=-\frac{\mathbf{x} \times \mathbf{I}}{4 \pi|\mathbf{x}|^{3}}
$$

where $\mathbf{I}=\frac{1}{2} \int \mathbf{y} \times \xi(\mathbf{y}) d \mathbf{y}$ is the impulse as defined in [2]. Therefore the flow due to the localized vorticity distribution is

$$
\mathbf{u}(\mathbf{x})=\nabla \times \frac{\mathbf{I} \times \mathbf{x}}{4 \pi|\mathbf{x}|^{3}}=\frac{1}{4 \pi|\mathbf{x}|^{3}}\left[\frac{3 \mathbf{x}(\mathbf{x} \cdot \mathbf{I})}{|\mathbf{x}|^{2}}-\mathbf{I}\right],
$$

which is the flow induced by a vortex dipole [22]. Note that if $\xi(\mathbf{x})=\nabla \times \mathbf{m} f_{\delta}(\mathbf{x})$, the equation above becomes

$$
\mathbf{u}(\mathbf{x})=\frac{1}{4 \pi|\mathbf{x}|^{3}}\left[\frac{3 \mathbf{x}(\mathbf{x} \cdot \mathbf{m})}{|\mathbf{x}|^{2}}-\mathbf{m}\right]
$$

which is exactly the impulse flow described by Eq. (4.2) outside the support of $f_{\delta}$. This analysis is valid for any localized vorticity distribution and for the evaluation point $\mathbf{x}$ in the 
far field. One concludes that far away from any localized vorticity distribution, the flow is that of a vortex dipole. In particular, if the vorticity is confined to a closed thin loop in the $x y$-plane with circulation $\Gamma$, then

$$
\mathbf{m}=\frac{1}{2} \int \mathbf{y} \times \xi(\mathbf{y}) d \mathbf{y}=\frac{\Gamma}{2} \oint \mathbf{y} \times d \mathbf{l},
$$

where $d I$ is a line element along the loop as shown in figure 6.1. Equation 6.2 implies that $\mathbf{m}$ is a vector perpendicular to the plane of the loop and with magnitude $|\mathbf{m}|=A \Gamma$, where $A$ is the area inside the loop (see figure 6.1).

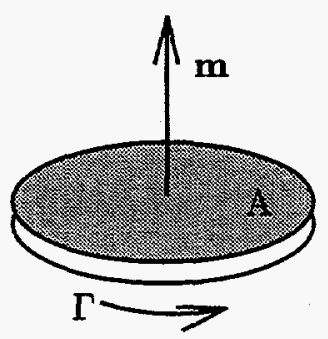

Figure 6.1: The impulse vector $\mathbf{m}$ induces the same flow as the vortex loop if $|\mathbf{m}|=A \Gamma$, where $A$ is the area enclosed by the loop and $\Gamma$ is the circulation around the loop.

\subsection{Comments}

A large thin vortex loop can be represented by a collection of many small loops lying on a surface that spans the large loop as in figure 6.2. Each of the small loops can be thought of as an impulse vector normal to the plane of the loop and with magnitude equal to the surface area enclosed by the loop multiplied the circulation around it. Impulse, $\mathbf{m}$, is thus a variable that localizes vorticity.

The non-uniqueness of $\mathbf{m}$ is evident in this interpretation of impulse since there are infinitely many surfaces that span a particular vortex loop. By construction, each surface is an m-orthogonal surface for the impulse field defined on it. Given a large, thin vortex loop that does not lie in a plane, the surface which requires the least number of impulse vectors of a prescribed size in its discretization is a surface with minimal area. The problem of finding the optimal impulse field to represent the flow induced by an arbitrary vortex loop is thus connected with finding minimal surfaces attached to a frame. 


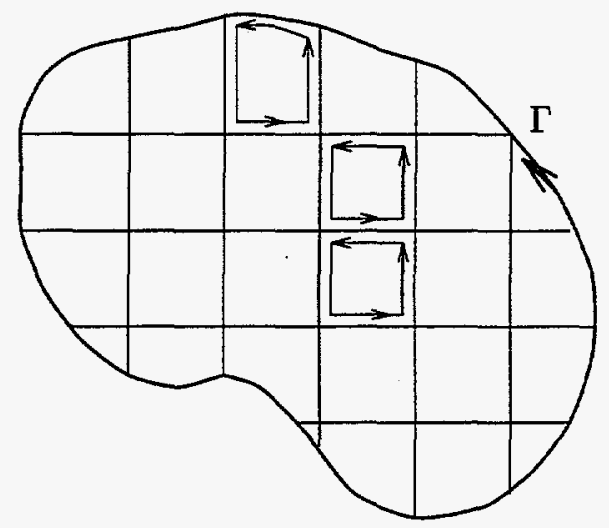

Figure 6.2: A large vortex loop represented as a collection of small loops.

A three-dimensional version of the impulse method with resurfacing presented in the previous chapter requires a recipe for collapsing the small loops represented by individual impulse vectors to the large loop. Then, one must define a new surface and the new impulse field on it. In two dimensions the new surface was a piece-wise linear curve; in three dimensions, the recipe for finding the minimal surface will not be presented here but is left for future work.

It was pointed out in chapter 3 that the magnitude of the an impulse vector is proportional to the area of a piece of the $\mathbf{m}$-orthogonal surface. That result is made obvious by Eq. (6.2) and the fact that the circulation around a vortex loop carried by incompressible flow is a constant of the motion [16]. In the case of a large vortex loop with unit circulation, the quantity $\sum\left|\mathbf{m}^{j}\right|$ is a measure of the area of the $\mathbf{m}$-orthogonal surface which is moving with the flow. As observed in [14] and seen in the example of $\$ 2.3 .2$, this quantity increases in time even when the total impulse is conserved.

\subsection{Examples}

This section illustrates the implementation of the impulse method presented by Buttke for three-dimensional flows. Two examples of flows with closed, circular vortex lines are presented. In the first example, the vorticity is confined to a sphere; the second one is a traditional vortex ring. The cutoff function used in both cases is the fourth-order function 
$f_{\delta}(r)=\delta^{-3} f_{1}(r / \delta)$, where $f_{1}(r)=\frac{3}{8 \pi}\left(5-3 r^{3}\right) e^{-r^{3}}[3]$. Numerical examples of the impulse method with refinement and resurfacing will be presented in the next sections.

\subsubsection{Hill's Spherical Ring}

The first example, presented in [2], is one in which the support of the vorticity is a ball of radius $a$ which translates in space but remains invariant otherwise. The vorticity in cylindrical coordinates $(r, \theta, z)$ is given by

$$
\xi(r, \theta, z)=(0, A r, 0)
$$

where $A$ is a constant. The vorticity is normal to the $z$-axis and constant on cylindrical shells lying within the ball. The same flow in terms of impulse can be written as

$$
\mathbf{m}(r, \theta, z)=\frac{A}{2}\left(0,0, a^{2}-z^{2}-r^{2}\right)
$$

for $(r, \theta, z)$ inside the sphere. The constant of integration has been chosen so that $\mathbf{m}(\mathbf{x}) \equiv \mathbf{0}$ for $|x| \geq a$. Since the support of impulse density and the support of vorticity are identical in this case, the impulse method is as efficient as a numerical method based on vorticity. Figure 6.3 shows one of the initial $\mathrm{m}$-orthogonal surfaces for a sphere of unit radius and $A=2$. The top figure shows several circles on the surface of the sphere marking latitudes at fixed vertical increments. The bottom picture shows the initial impulse field on one surface.

The flow induced by the vorticity distribution of Eq. (6.3) is steady and can be computed analytically [2]. The velocity field relative to axes moving with the sphere is

$$
\mathbf{u}(r, \theta, z)=\frac{2}{5}\left(r z, 0,1-z^{2}-2 r^{2}\right)
$$

From this expression, it is easy to see that a particle on the surface of the sphere, say at a point $(r, \theta, z)$ with $r^{2}=1-z^{2}$, moves along the surface of the sphere with speed $2 r / 5$ in the direction away from the the point $(0,0,1)$, the north pole. Consequently, the latitudinal curves in figure 6.3 will drift downward on the surface of the sphere. The curves near the equator will move faster than those near the poles. Figure 6.4 shows the same curves and surface as the previous figure but at time $t=1.2$. The cutoff radius was fixed at $\delta=0.35$ and the discretization size was set to about 0.18 . The $\mathbf{m}$-orthogonal surfaces passing through the center of the sphere swell up faster than others since the flow is fastest 

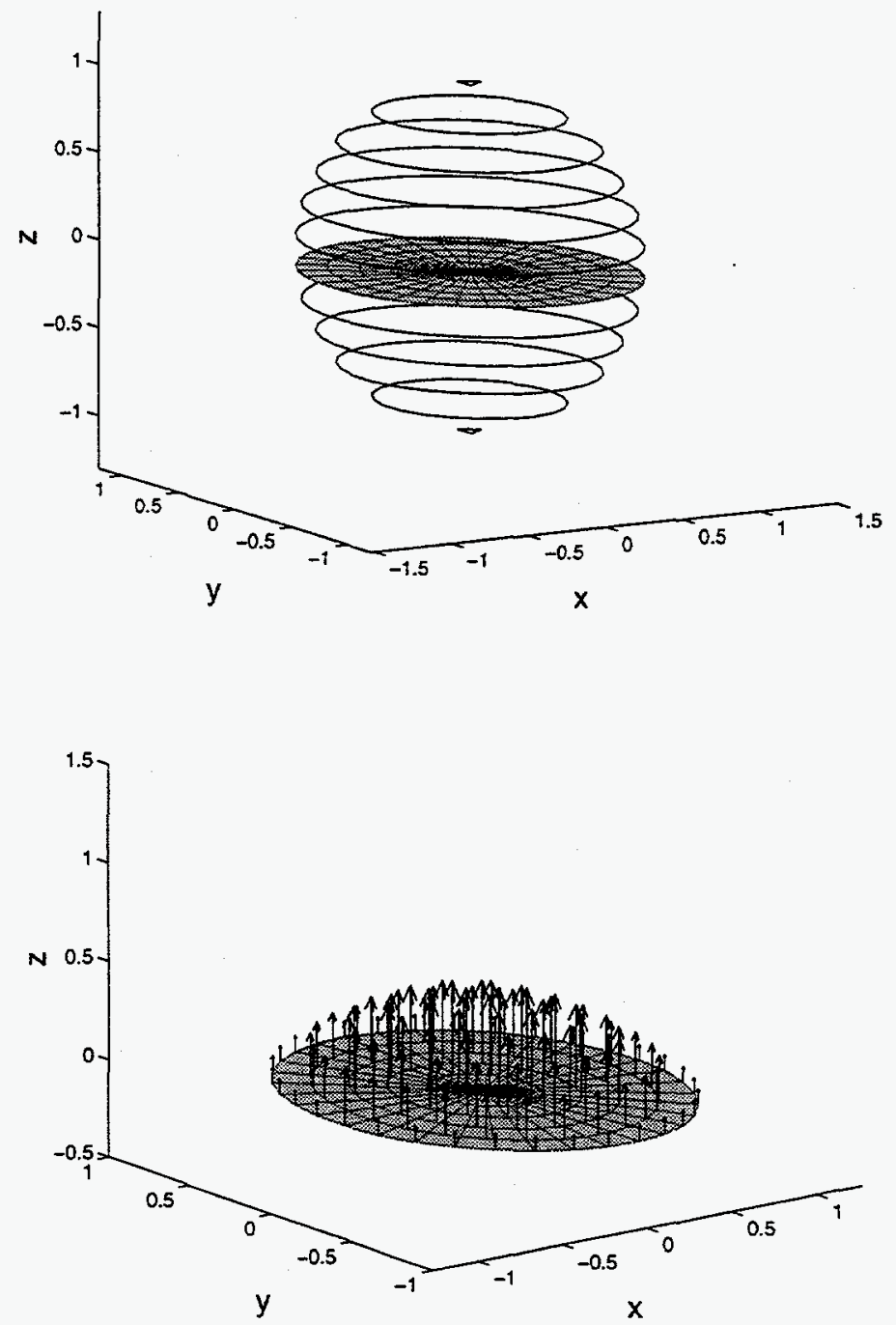

Figure 6.3: Latitudinal curves on the initial sphere. The initial impulse is defined on flat surfaces like the one shown in the top picture. The bottom picture shows the impulse on one $\mathbf{m}$-orthogonal surface at time $t=0$. 

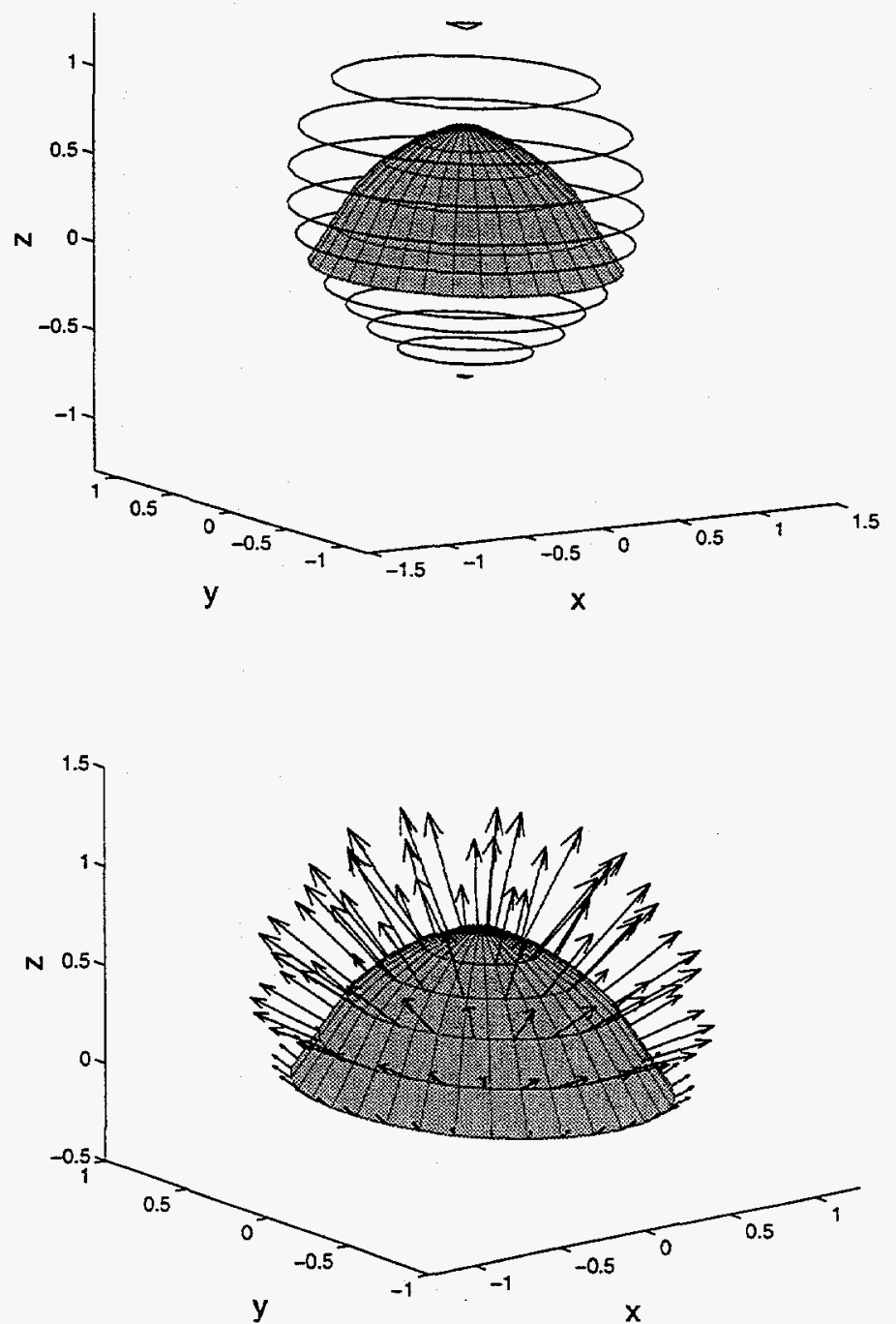

Figure 6.4: Latitudinal lines on the sphere at $t=1.2$. The m-orthogonal surfaces swell up. The bottom picture shows the impulse on one $\mathbf{m}$-orthogonal surface at time $t=1.2$. 
at the center of the sphere. The set of stagnation points includes the north and south poles, so the m-orthogonal surfaces near the poles swell up slowly. A front view of the latitudinal curves in figure 6.4 is shown in figure 6.5 for a better appreciation of their downward motion. One can see here that the sphere has translated in the positive $z$-direction while the circles on the surface have migrated down.
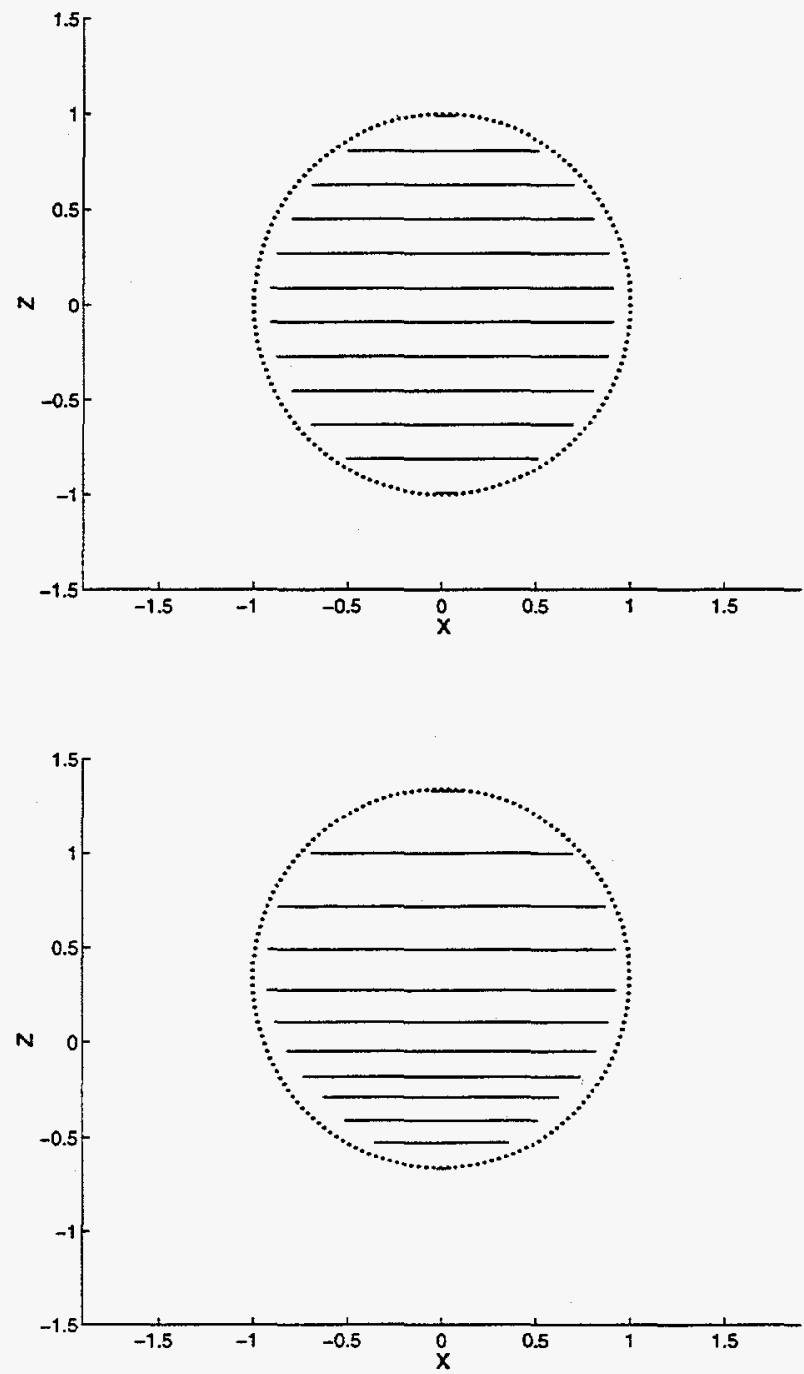

Figure 6.5: Front view of the latitudinal lines on the surface of the sphere at time $t=0$ (top) and time $t=1.2$ (bottom). The lines travel away from the north pole. 


\subsubsection{A Vortex Ring}

While the vorticity distribution in the previous example may be considered a vortex ring, a more traditional vortex ring in which the vorticity is confined to a torus of radius $R$ and core radius $a$ with $a<R$, is presented in this section. The impulse density that defines a vortex ring of constant vorticity $\xi(r, \theta, z)=(0, \omega, 0)$ is $\mathbf{m}(r, \theta, z)=\left(0,0, m_{3}\right)$ where

$$
m_{3}(r, \theta, z)= \begin{cases}\omega\left[R+\sqrt{a^{2}-z^{2}}-r\right], & \text { for }(r-R)^{2}+z^{2} \leq a^{2} \\ \omega \sqrt{a^{2}-z^{2}}, & \text { for } z^{2} \leq z^{2} \text { and } r \leq R-\sqrt{a^{2}-z^{2}} .\end{cases}
$$

Note that the impulse is continuous while the vorticity is not. The impulse density of this example cannot be restricted to the torus [7] since the circulation around a loop which encircles the ring but lies outside of the support of the vorticity is $\pi a^{2} \omega$. As a consequence, more particles are needed in this case for the representation of the ring by impulse than by vortex elements.

Figure 6.6 shows the initial ring for $R=0.5, a=0.2$ and vorticity with $\omega=1 / 2 a$ so that $m_{3}(0,0,0)=1$. The m-orthogonal surfaces are horizontal discs as in figure 3.7 in chapter 3 .

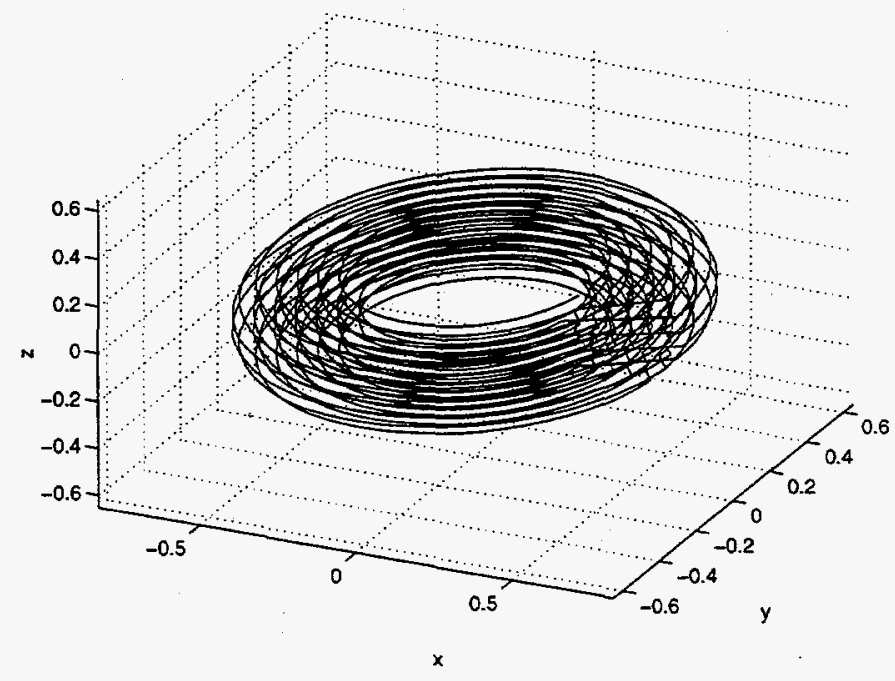

Figure 6.6: Initial vortex ring of radius $R=0.5$ and core radius $a=0.2$.

The impulse density was approximated by points on a grid which is nearly uniform in cylindrical coordinates with spacing $h=0.06$. The cutoff radius was set at $\delta=0.3$. The ring at time $t=4$ is shown in figure 6.7. At this time the core has rotated about one half 


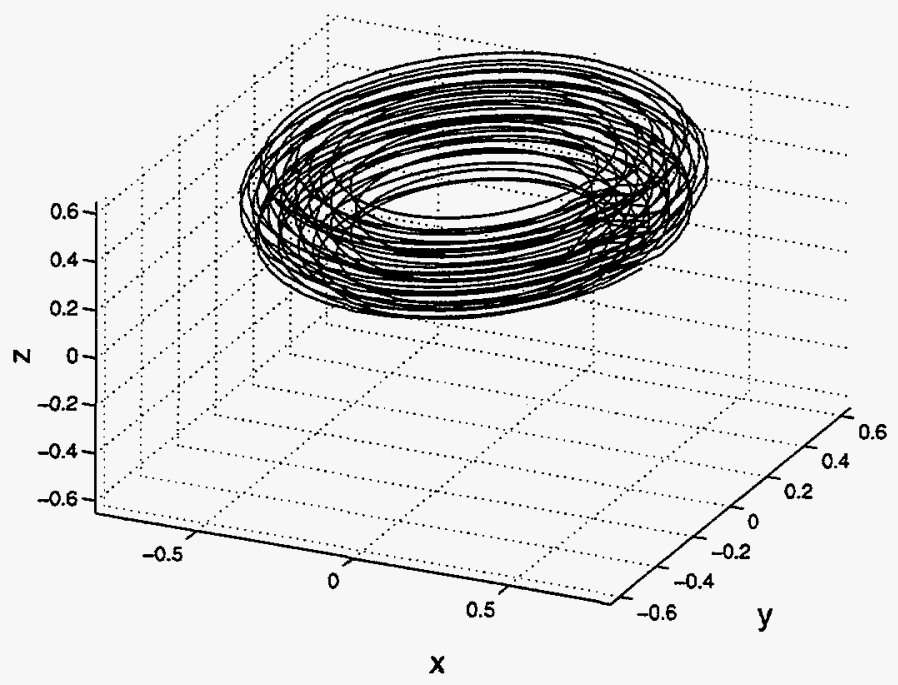

Figure 6.7: Vortex ring of radius $R=0.5$ and core radius $a=0.2$ at time $t=4$.

of a revolution and the m-orthogonal surfaces are swollen due to the fluid ejected through the ring. Figure 6.8 shows one of these surfaces at various times in the evolution of the flow. By $t=2$, the impulse vectors on the top surfaces had stretched by a factor of 3 near the center of the ring. By $t=4$, the same vectors had stretched by a factor of 8 to 11 , indicating that the impulse density is no longer well approximated in those regions.

It is important to stress that the energy, impulse, and angular momentum have been kept invariant to at least 8 significant digits throughout the computations in both examples of this chapter. As in two dimensions, the conservation of these invariants will continue as the method solves accurately the Hamiltonian system of dipoles. The question of how long those dipoles represent the flow induced by the vortex ring is a separate one.

\subsection{Impulse Method With Refinement}

A three-dimensional analogue of the refinement procedure from last chapter can be designed now that the connection between impulse vectors and vortex loops has been established. One thinks of a vector $\mathbf{m}$ located at the point $\mathbf{x}$ as an object that induces the flow of a vortex loop centered at $\mathbf{x}$, lying on the plane normal to $\mathbf{m}$, and having circulation $\Gamma$ and area $A$. It is now known that the relation $|\mathbf{m}|=A \Gamma$ holds. A refined representation of the same flow is given by smaller loops which span the former one and whose circulation 

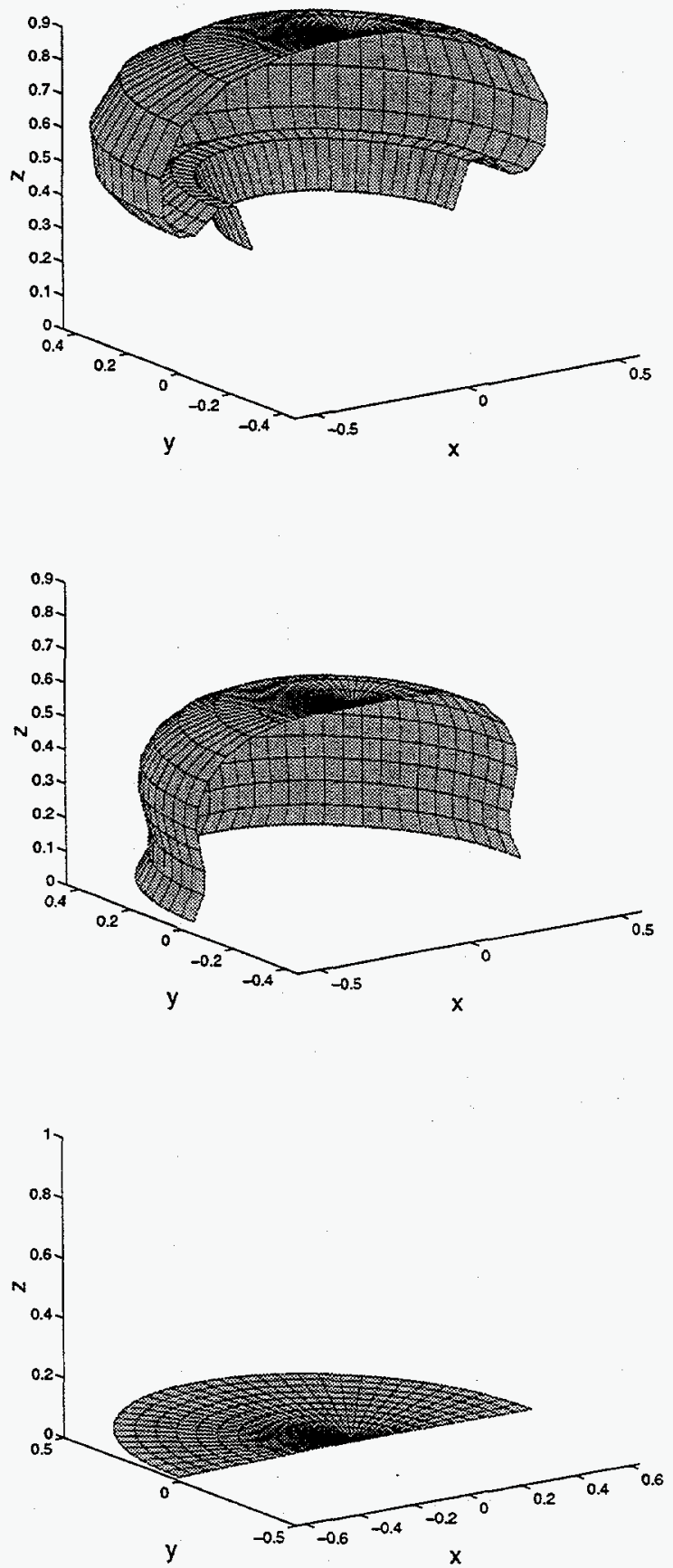

Figure 6.8: Figures showing half of an $\mathbf{m}$-orthogonal surface located initially on the $x y$ plane. The surface is shown at times $t=0$ (bottom), $t=2$ (middle) and $t=4$ (top). 
is still $\Gamma$. If one chooses vectors $\mathbf{m}_{1}, \mathbf{m}_{2}, \cdots, \mathbf{m}_{L}$ to represent the smaller loops, then the conditions

$$
\sum_{k=1}^{L}\left|\mathbf{m}_{k}\right|=A \Gamma, \quad \sum_{k=1}^{L} \mathbf{m}_{k}=\mathbf{m}
$$

will enforce incompressibility and ensure the invariance of impulse and circulation around the original loop. In contrast to the two-dimensional case, the shape of the loops is arbitrary, and so there is no unique way to subdivide a loop. For instance, one can consider a loop of area $A$ centered at $\mathbf{x}$ and subdivide it into 4 loops of area $A / 4$ located on the corners of a square of side $\sqrt{A} / 2$ as shown in figure 6.9. This convention is adopted here.

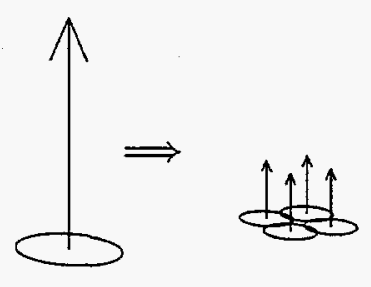

Figure 6.9: Refinement of impulse in three dimensions. The impulse vector on the left induces the flow of a vortex loop of area $A$. The refinement process replaces the vector by four vectors of magnitude $1 / 4$ the original one.

Consider a vortex ring of radius $R=1$ and core radius $a=0.4$. As noted before, the surfaces at the top of the ring are the first ones to suffer the most stretching so an morthogonal surface initially at $z=0.32$ is presented in figure 6.10 . The surface is shown as a collection of loops at $t=4$ both without refinement (top) and with refinement (bottom). The size of each loop is proportional to the size of the vector it represents. The ring is initialized by points on a cylindrical grid of size $h=0.16$ and the cutoff radius is $\delta=0.35$. These parameters are chosen merely for the illustration of the refinement procedure. The criterion used for the refinement is the area spanned by the loop being 4 times its original value. Once the subdivisions occur, each vector evolves independently giving more flexibility to the surface. The energy is conserved to within $4 \%$ of its initial value throughout the computation. Note that some of the loops have not met the criterion yet while others have already been subdivided. It is also evident that the loops run into each other in the azimuthal direction but show gaps on rays of constant angle. This is an indication that the surface does not stretch equally in all directions but favors directions of constant $\theta$. This kind of information is helpful in deciding what refinement criteria to use. For this problem, 
one may want to subdivide each loop into 2 loops centered along the intersection of the plane of the loop with the planes of constant $\theta$.
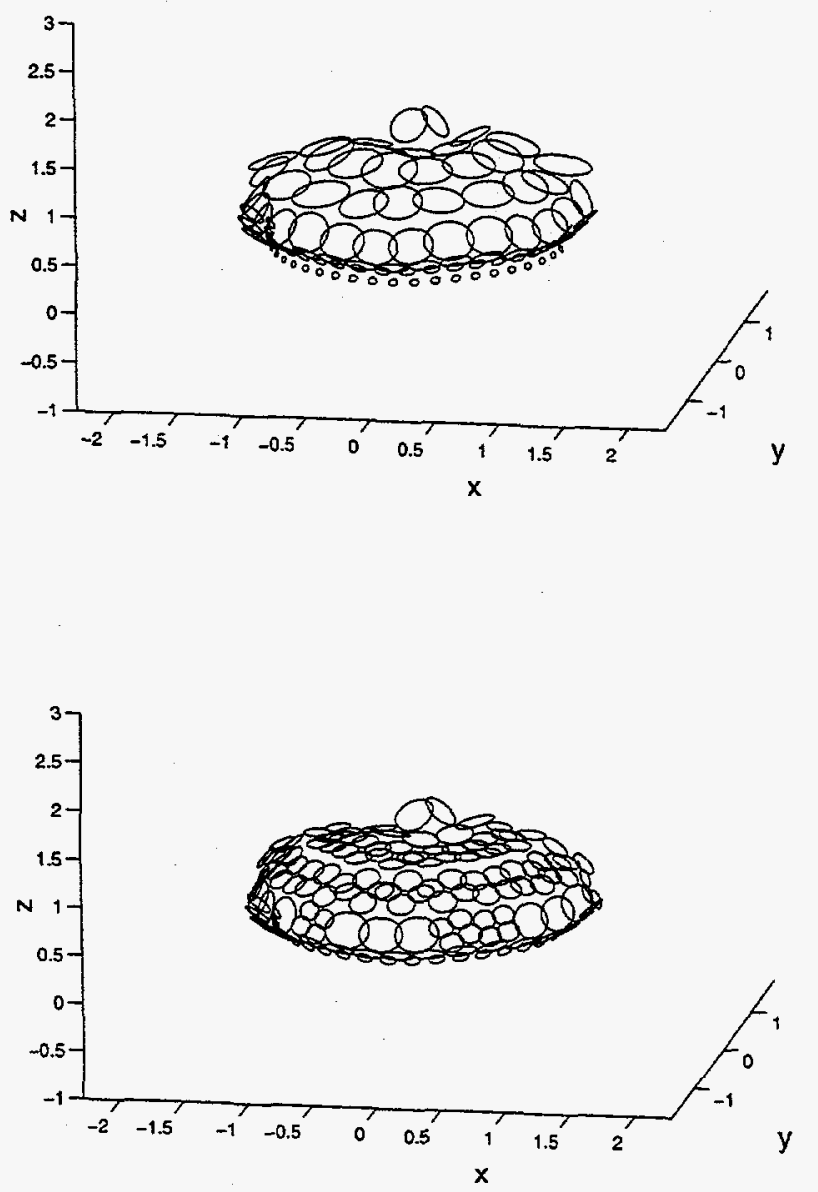

Figure 6.10: Vortex loops representing an m-orthogonal surface for a ring of $R=1$ and $a=0.4$ at time $t=4$. The top picture shows the loops without refinement; the bottom picture shows the refinement of some loops while others have not yet been refined.

\subsection{Impulse Method With Resurfacing}

Consider a large, thin vortex loop and an impulse field that represents the flow induced by the loop. This impulse is defined on a surface which spans the loop. The most efficient impulse representation of this flow would be one in which the impulse is defined 
on a minimal surface bounded by the loop. Thus a general resurfacing procedure would take a collection of impulse vectors as input and would (1) determine the vortex loop they represent, (2) compute a minimal surface that spans the loop, and (3) define a new impulse field on the minimal surface.

In two dimensions, each impulse vector was replaced by two vortices whose positions were adjusted to exploit cancellation of vorticity. In three dimensions, each impulse vector represents a small vortex loop of arbitrary shape lying on a plane normal to the vector and enclosing a given area. The situation is more intricate here since the arbitrariness of the small loops makes it difficult to determine whether two adjoining small loops can be combined to form a larger one. Additionally, the computation of a minimal surface attached to a frame is a complex mathematical task in itself [10]. Some frames allow topologically different minimal surfaces. From the point of view of impulse variables, the resurfacing procedure should produce a surface whose area is smaller than the area of the surface being replaced but not necessarily a minimal surface. One with nearly minimal area may be sufficient for the definition of the new impulse field and may avoid some of the high computational cost of reaching the minimal surface.

A general resurfacing procedure is left for future work and will not be presented at this time. However, the particular case of the smooth vortex ring of the previous section will be used as an illustration of the resurfacing procedure. In this case, the initial morthogonal surfaces are flat discs where the impulse defined on each surface represents the flow of several concentric circular vortex loops lying on that surface. One can make use of the fact that the vortex loops remain circular and flat as the flow evolves, so that one point on the loop is enough to determine the location of the entire loop. A threading routine similar to the one in two dimensions is used here to connect several loops with one surface in order to keep the number of surfaces (and therefore impulse vectors) small. Figure 6.11 shows $m$-orthogonal surfaces at time $t=1$ before and after the resurfacing. Note that the new surface does not necessarily represent the same vortex loops as the old surface, but the new surfaces together do represent all vortex loops and have smaller area than the surfaces being replaced. As a consequence, the new impulse field consists of vectors of smaller magnitude than the replaced vectors and an equal number of vectors can be used to represent the flow with higher accuracy. 

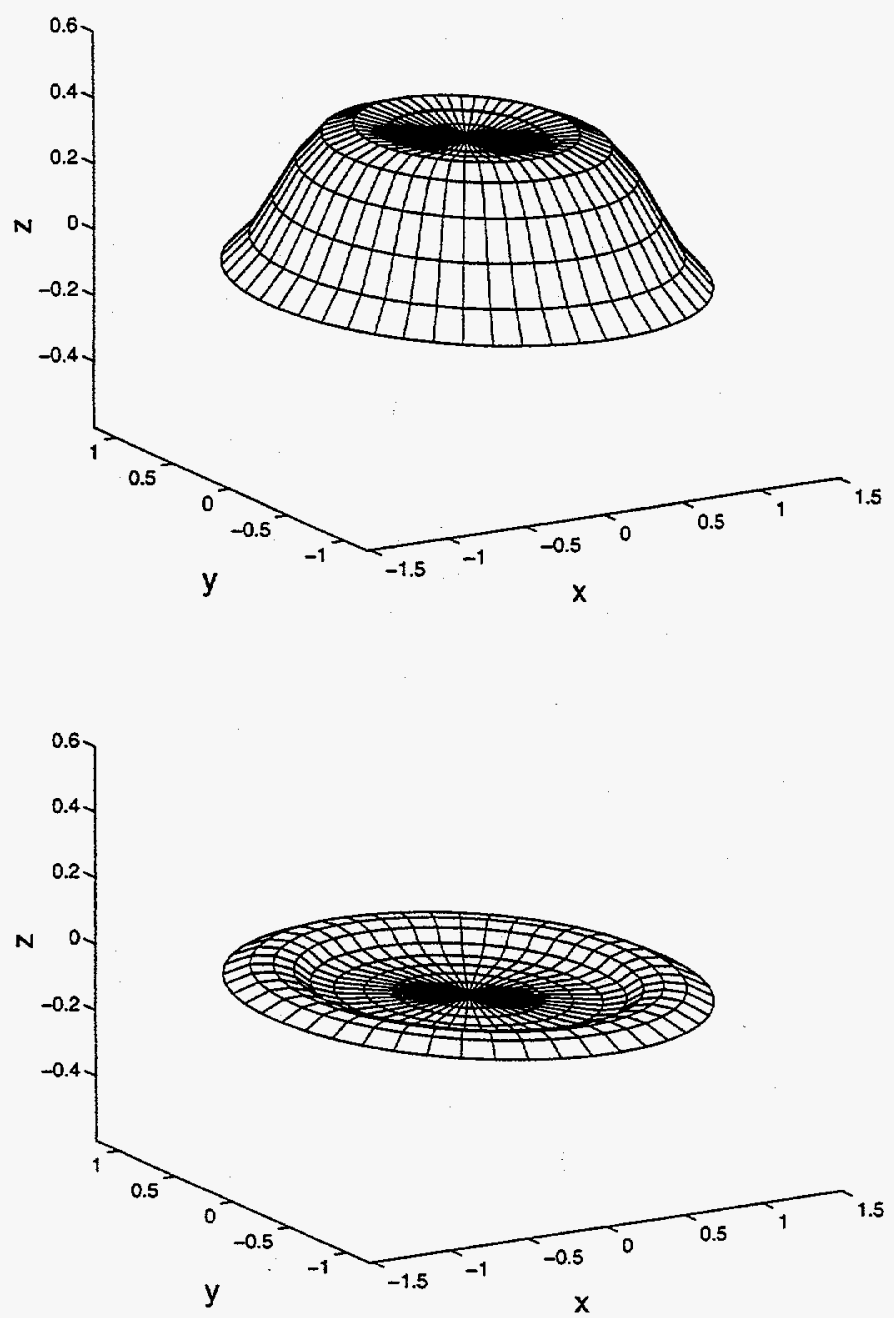

Figure 6.11: $\mathrm{m}$-orthogonal surfaces for a vortex ring at time $t=1.0$. The top figure shows one of the original surfaces and the bottom figure shows a new surface after the resurfacing procedure. 


\section{Chapter 7}

\section{Application of Impulse Methods to Immersed Boundary Problems}

The previous chapters address the approximation of vorticity fields by impulse and techniques for maintaining the accuracy of these approximations over time. This chapter discusses applications of impulse methods to problems for which these methods are particularly well-suited. Consider the example of a flow in which a force acts on immersed boundaries, like the interface between two fluids or an elastic membrane containing a fluid. Since force is by definition a rate of change of impulse, it is natural to represent the effect of forces acting on particular regions of the flow by introducing an impulse field which changes according to the Navier-Stokes equations with an additional force term.

Interesting situations of a fluid in a region bounded by an elastic membrane include air flow in the lungs, blood flow through the heart, and water sloshing inside a balloon. The key feature common to these examples is the interaction between the elastic forces on the boundary and the fluid inside and outside. As the membrane stretches and contracts, the forces will affect the motion of the fluid and this motion will alter the configuration of the boundary, which determines the forces.

Previous numerical treatments of this type of force-fluid interaction use vortex elements on the boundary to introduce the effect of the forces at every time step. The elements created dynamically on the boundaries are vortex pairs approximating dipoles [27] or a combination of vortex pairs and simple vortex layers [26]. The discussion in chapter 4 about the equivalence of impulse vectors and vortex dipoles makes it evident that vortex- 
pair approximations of dipoles are no longer necessary and that the strengths of the dipoles are updated correctly by the impulse equation of motion. Since boundary forces naturally interact with the fluid by imparting impulse, the impulse method is most appropriate for the numerical solution of this type of problem. The first use of impulse methods in this setting is found in [17], where the motion of an inviscid, incompressible fluid confined to a region with elastic boundary was computed.

The following sections contain the discussion of boundary forces and energy calculation for the case of inviscid fluids, and present numerical examples. The final sections show a method for introducing viscous effects into the model and more numerical results.

\subsection{The Formulation of the Problem}

The application presented here is the motion of a two-dimensional incompressible fluid in free space and an elastic membrane surrounding a bounded region of fluid (see figure 7.1). The assumption that the same fluid occupies the region outside the membrane

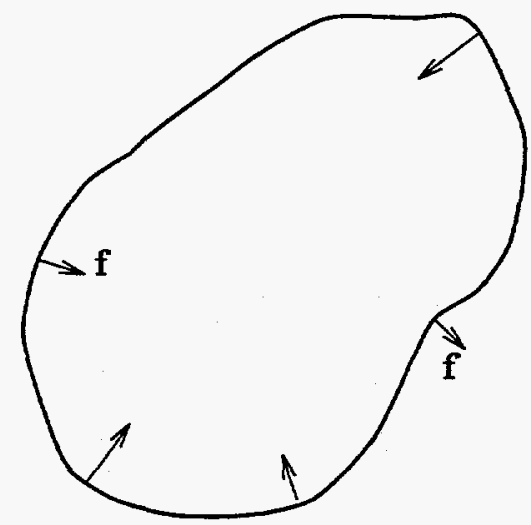

Figure 7.1: Initial setup of an elastic membrane surrounding a fluid.

is made to focus on the motion due to the elastic forces and not due to density changes across the membrane. This assumption is reasonable in physiological applications such as the blood flow in the heart chambers, where blood also occupies regions outside the chambers [26]. The membrane is idealized as being infinitely thin and massless so that the force field is concentrated only on the curve defined by the membrane. The forces are therefore singular since they act on a set whose dimension is lower than the spatial dimension of the problem. 
One may think of the membrane as a string of small rubber bands linked to their two nearest neighbors. The force exerted on the fluid by the membrane depends solely on the configuration of the membrane, and each point on the membrane is assumed to move with the local fluid velocity.

\subsection{Boundary Forces}

The Navier-Stokes equations are

$$
\frac{D \mathbf{u}}{D t}=-\nabla p+\nu \Delta \mathbf{u}+\mathbf{F}, \quad \nabla \cdot \mathbf{u}=0
$$

where $\mathbf{F}$ represents external force. The same derivation of the impulse equations from chapter 2 leads to

$$
\begin{aligned}
\frac{D \mathbf{m}}{D t} & =-(\nabla \mathbf{u})^{T} \mathbf{m}+\nu \Delta \mathbf{m}+\mathbf{F} \\
\mathbb{P m} & =\mathbf{u},
\end{aligned}
$$

where it is clearly displayed that force is a rate of change of impulse. The numerical method consists of solving Eq. (4.4)-(4.5) with the addition of the force term in the second equation. Let $\mathrm{f}^{j}$ be the force on the piece of boundary represented by the $j$-th particle, then the equations of motion for the particle locations and the impulse strengths in the case of inviscid flows $(\nu=0)$ are

$$
\begin{aligned}
\frac{d \mathbf{x}^{j}}{d t} & =\mathbf{u}\left(\mathbf{x}^{j}\right) \\
\frac{d \mathbf{m}^{j}}{d t} & =-(\nabla \mathbf{u})^{T} \mathbf{m}^{j}+\mathbf{f}^{j}
\end{aligned}
$$

Let $s$ be the arclength parameter and $\mathbf{x}(s)$ a parametrization of the membrane, then the forces exerted by the elastic membrane on the fluid can be written as [26]

$$
\mathbf{F}(\mathbf{x})=\int \mathbf{f}(s) \delta(\mathbf{x}-\mathbf{x}(s)) d s
$$

where $f$ is the force density, and $\delta$ is the two-dimensional Dirac delta function. Note that $\mathbf{F}$ is singular since the integral is taken along a curve and $\delta$ is two-dimensional. A different approach to evaluating the force term can be found in [9].

The force density is of the form $\mathbf{f}(s)=d(T \hat{\tau}) / d s$, where $T$ is tension, and $\hat{\tau}$ is the unit vector tangent to the boundary $[30,17]$. Expanding the derivative in the definition of 
force density, one obtains

$$
\mathbf{f}(s)=\frac{d(T \hat{\tau})}{d s}=\frac{d T}{d s} \hat{\tau}+T \frac{d \hat{\tau}}{d s}=\frac{d T}{d s} \hat{\tau}+T \kappa \hat{\mathbf{n}}
$$

where $\kappa$ is the mean curvature and $\hat{\mathbf{n}}$ is the outward unit vector normal to the boundary. The distinction between inviscid and viscous flows is relevant at this point in the computation of forces. In Euler flow, the tangential forces simply make the boundary slip so that the only force affecting the motion of the fluid is the one normal to the membrane, i.e.

$$
\mathbf{f}(s)=T \kappa \hat{\mathbf{n}} .
$$

In this case the slip condition is enforced by setting $\frac{d T}{d s}=0$, which states that the tension is constant along the membrane at any instant. This condition must be enforced in the numerical method. For viscous flows, the normal and tangential forces will affect the fluid motion and Eq. (7.4) must be used.

In practice, the forces are found at the locations of the impulse vectors. The force associated with a piece of boundary of length $h^{j}(t)$ corresponding to the the discretization size of the $j$-th particle is $\mathbf{f}^{j}=\mathbf{f}\left(\mathbf{x}^{j}\right) h^{j}(t)$. This is the force used in Eq. (7.2).

Each piece of arclength (the discretization size) is thought of as a rubber band with a stiffness constant and a rest-length. Denote by $h_{0}$ the rest-length of all elements, and define the tension at $\mathbf{x}^{j}$ by the equation

$$
T\left(\mathbf{x}^{j}\right)= \begin{cases}\sigma\left(h^{j}(t)-h_{0}\right) / h_{0}, & h^{j}(t)>h_{0} \\ \mathbf{0}, & h^{j}(t) \leq h_{0}\end{cases}
$$

where $\sigma$ is a stiffness constant. Note that there are no forces when the elements are compressed below $h_{0}$. In the case of Euler flow, the constant tension along the membrane requires that the particles be equally distributed on the membrane at every time step. This is accomplished with the use of a cubic polynomial interpolant [17]. The curvature at every point is also found using a cubic polynomial, which is chosen to go through three points on the membrane and to match the slope at the middle point. The curvature of the membrane at the middle point is approximated by the curvature of the polynomial.

\subsection{The Membrane Algorithm}

The algorithm used for the tracking of the membrane consists of three parts at every time step $\Delta t$ : 
- Based on the current boundary configuration, compute the forces and update impulse

$$
\mathbf{m}^{j} \longleftarrow \mathbf{m}^{j}+\Delta t \mathbf{f}^{j}
$$

This part assumes that the force is constant throughout the time step. This assumption has been found to be reasonable and attractive due to its simplicity [17].

- Update positions and strengths via Euler equations

$$
\begin{aligned}
\frac{d \mathbf{x}^{j}}{d t} & =\mathbf{u}\left(\mathbf{x}^{j}\right) \\
\frac{d \mathbf{m}^{j}}{d t} & =-(\nabla \mathbf{u})^{T} \mathbf{m}^{j} .
\end{aligned}
$$

These equations are solved using a fifth-order Runge-Kutta method.

- Add viscous effects if appropriate

$$
\mathbf{m}^{j} \longleftarrow \mathbf{m}^{j}+\Delta t \nu(\Delta \mathbf{m})^{j}
$$

The implementation of the this part will be discussed later in this chapter.

\subsection{Numerical Results for Inviscid Flow}

The initial setup consists of a membrane in a shape of a circular water balloon with a dimple on one side (see Figure 7.2). When the motion starts, the forces in the indented part of the boundary are stronger than other forces and make this portion move faster than the rest, creating waves which propagate along the boundary. The exact initial shape of the right side of the membrane is given by $x(\theta)=r(\theta) \cos (\theta)$ and $y(\theta)=r(\theta) \sin (\theta)$, where $r(\theta)=1-\frac{7}{10}\left(1-10 q^{3}+15 q^{4}-6 q^{5}\right)$ and $q=2 \theta / \pi$. The left side is the circle $r(\theta)=1$. The boundary was discretized using 300 equally spaced particles and the rest-length of all elements was set to half the initial discretization size. The stiffness of all elements was set to $\sigma=0.5$, the cutoff radius was $\delta=0.12$ and the cutoff function was $f_{\delta}(r)=\delta^{-2} f_{1}(r / \delta)$ where $f_{1}(r)=\frac{1}{2 \pi}\left(e^{-r^{2}}+\frac{1}{2} e^{-r^{2} / 2}\right)$.

The motion of the membrane for time 0 through 5.4 and $\Delta t=0.004$ is shown in figures 7.3 and 7.4. The initial impulse field was identically zero; what gets the motion started is the forces induced by the initial membrane configuration. The area bounded by the membrane must be conserved since the flow is incompressible, as well as the total energy, 


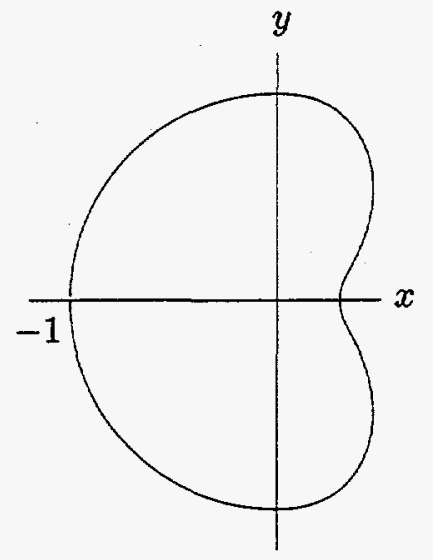

Figure 7.2: Initial membrane configuration.

kinetic plus potential. The kinetic energy of the fluid is given by $H=1 / 2 \sum \mathbf{m}^{j} \cdot \mathbf{u}\left(\mathbf{x}^{j}\right)$, as before. The potential energy in the elastic membrane is $E=\frac{\sigma}{2} \sum\left(h^{j}(t)-h_{0}\right)^{2} / h_{0}$ for $h^{j}>h_{0}$ and zero otherwise. The impulse is perpendicular to the membrane since the forces are always normal to the membrane. The total energy is given by the initial potential energy because the motion is started from rest. By conservation of energy, the potential energy never surpasses its initial value and so the length of the membrane is never larger than its initial length. This implies that the accuracy problems due to stretching of $\mathbf{m}$-orthogonal surfaces is not an issue in this application.

The invariants are conserved approximately in spite of the redistribution of particles at every time step. This procedure is equivalent to replacing the Lagrangian particles by a new set of variables at every step. In this example the energy was conserved to within $4 \%$ of its initial value and area to within $0.5 \%$ of its initial value for $0 \leq t \leq 5.4$ as seen in figure 7.5 .

The impulse vectors are shown at times $t=1.2$ and $t=5$ in figure 7.6. Since the forces on the right side of the membrane point in the outward direction for a small initial time interval, some of the impulse vectors still point outward at $t=1.2$. Because the impulse represents the accumulation of force over time and the forces act mostly in the inward direction, one expects the impulse vectors to have larger magnitudes for longer times. This is seen in the bottom picture of figure 7.6. 

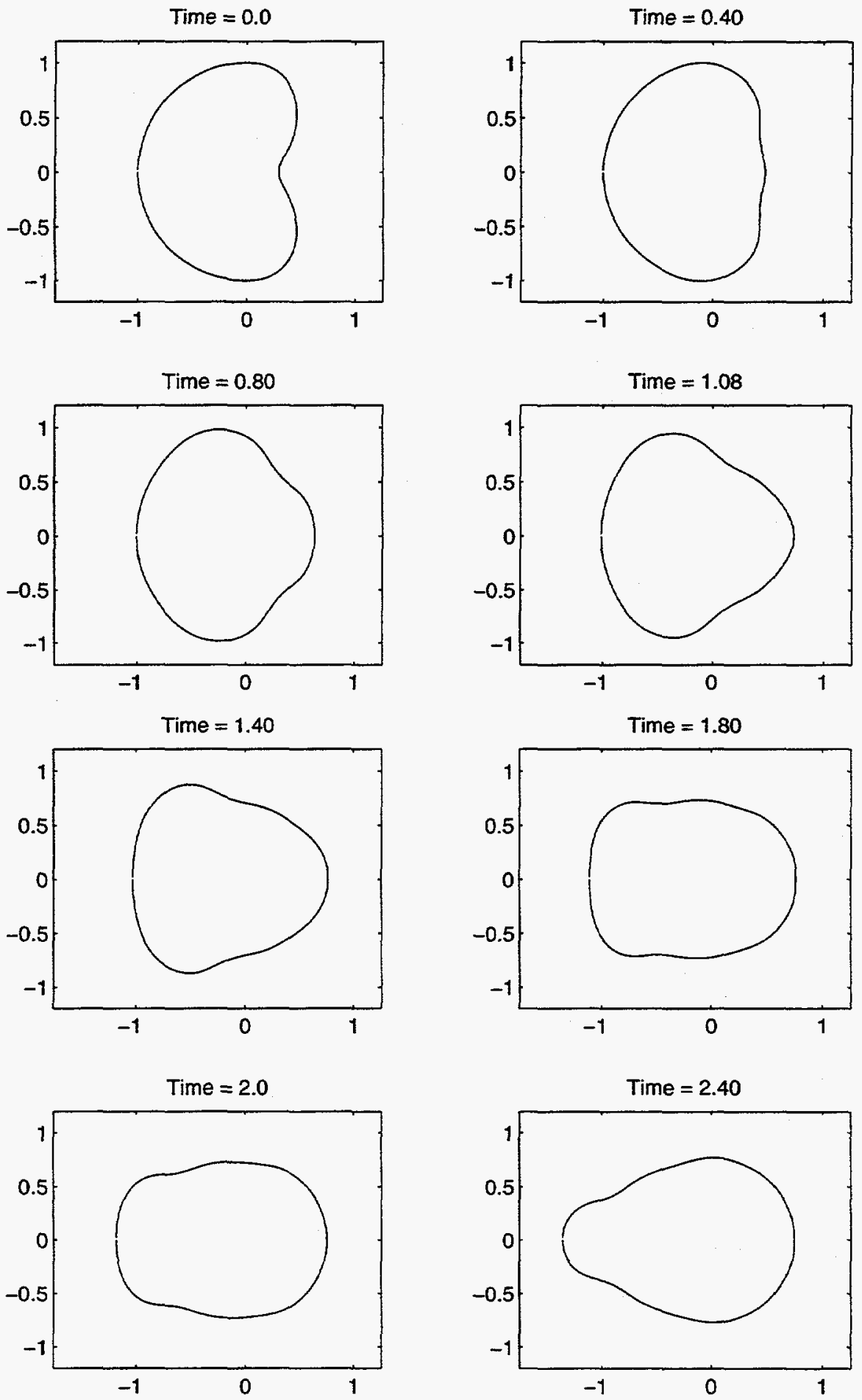

Figure 7.3: Motion of the membrane for time $t=0-2.4$, with $\Delta t=0.004$. 

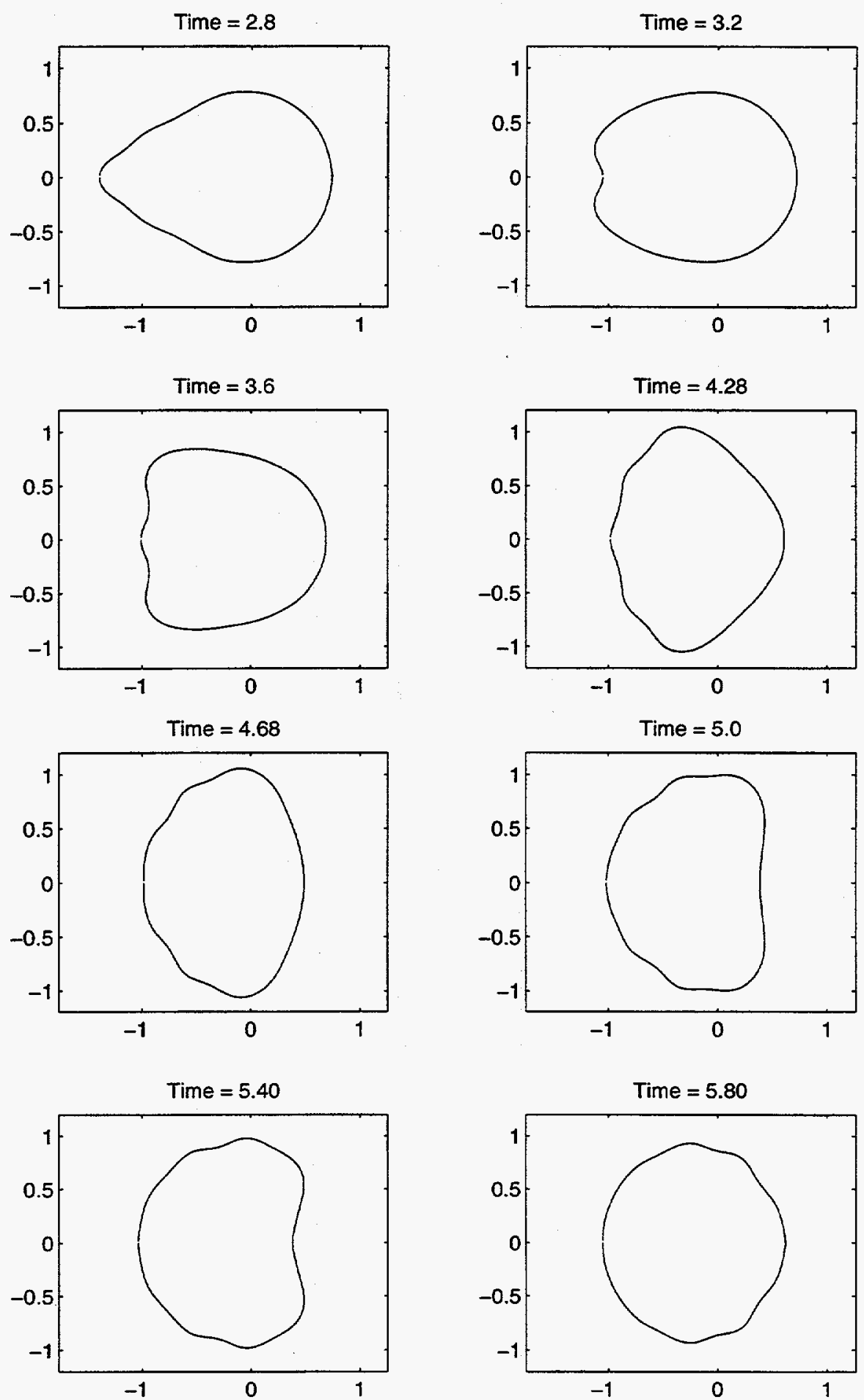

Figure 7.4: Motion of the membrane for time $\mathrm{t}=2.8-5.8$, with $\Delta t=0.004$. 

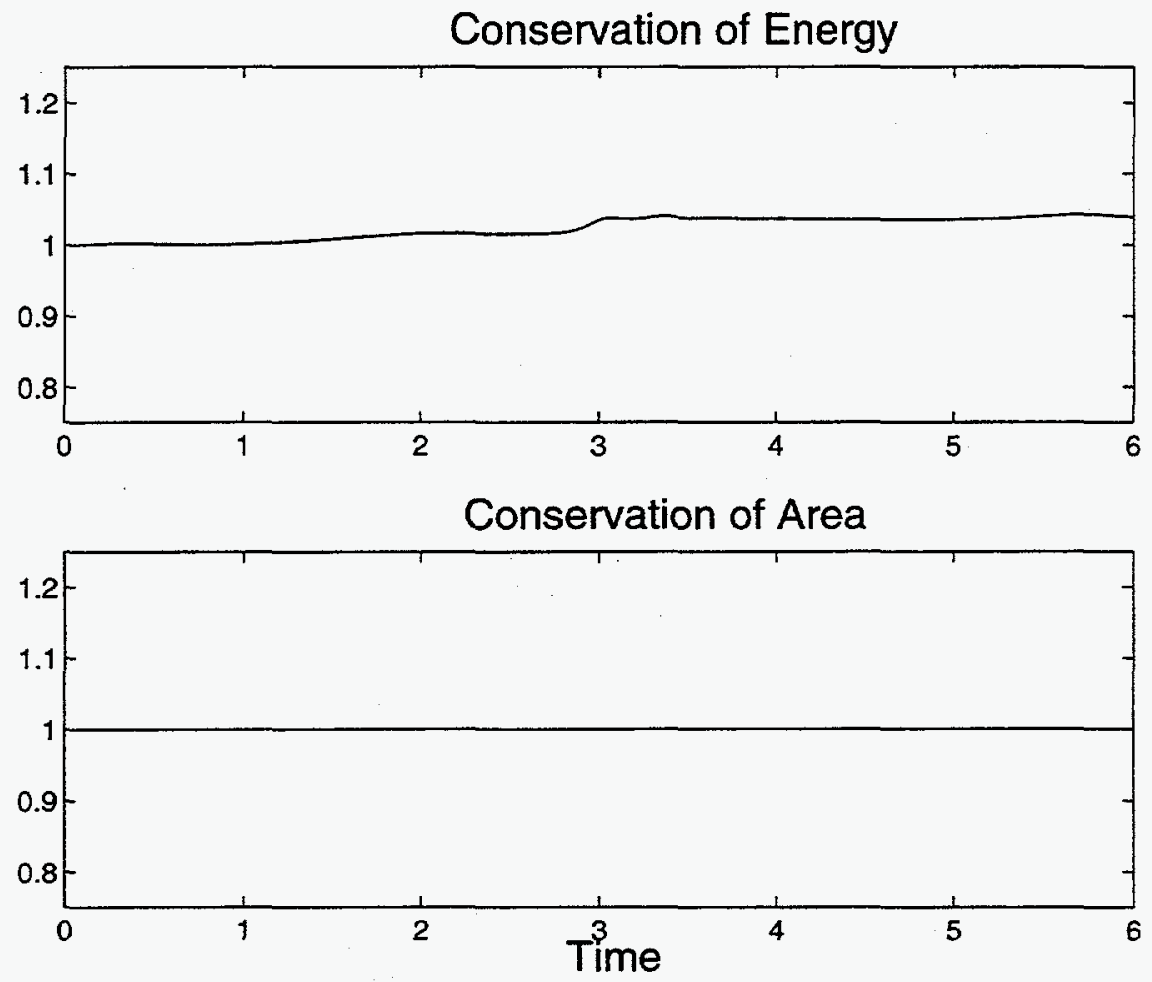

Figure 7.5: Approximate conservation of invariants for the inviscid problem. 

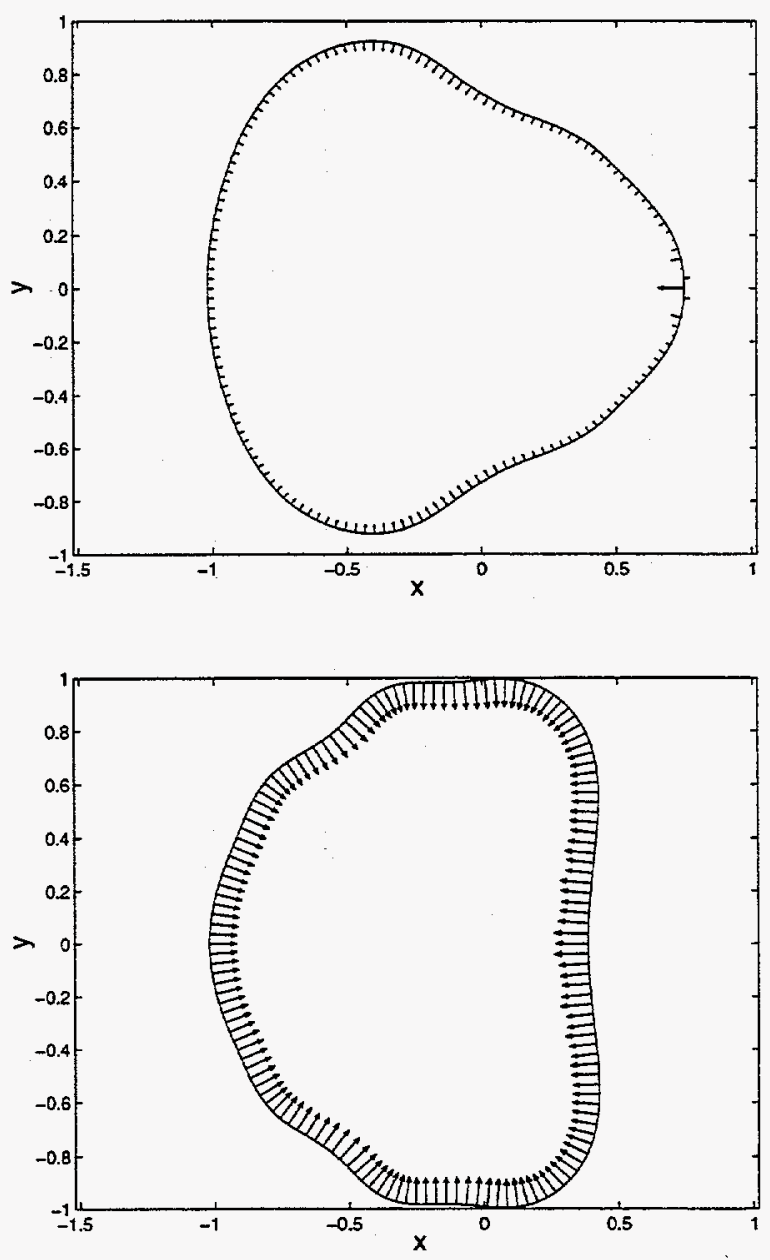

Figure 7.6: Impulse field at times $t=1.2$ (top) and $t=5.0$ (bottom). 


\subsection{The Diffusion Term}

In order to model viscous flows, one must approximate the diffusion term $\Delta \mathbf{m}$ in the equation of motion for impulse. One approach for the modeling of diffusion for vortex elements is to impose an appropriate random walk to the particles [13]. In the case of the elastic membrane, marker particles would hold the location of the membrane and new particles would have to be created at every time step to account for the diffusion. The position of the new impulse particles can have the effect of perturbing the membrane in ways that will produce large forces (due to large curvatures) in random directions, leading to instability. Therefore, a deterministic technique for the modeling of diffusion is adopted here. The approach is taken from [18] and consists of approximating the impulse by the convolution of it with a cutoff function of the same type used in the numerical method, though not necessarily the same function or cutoff radius. Then the derivatives in the Laplacian operator can be applied to the cutoff function,

$$
\Delta \mathbf{m} \approx \Delta\left(\mathbf{m} * f_{\epsilon}\right)=\mathbf{m} * \Delta f_{\epsilon}
$$

If the convolution is approximated by a sum, one can define the quantity

$$
(\Delta \mathbf{m})^{k}=A^{k} \sum_{j} \mathbf{m}^{j} \Delta f_{\epsilon}\left(\left|\mathbf{x}^{k}-\mathbf{x}^{j}\right|\right)
$$

where $\mathbf{m}^{j}$ are the same impulse strengths used in the numerical method and $A^{k}$ is the element of area associated with $\mathbf{m}^{k}$. The viscous effects can then be added by adjusting the impulse strengths according to $\frac{d \mathbf{m}^{k}}{d t}=\nu(\Delta \mathbf{m})^{k}$ at the end of the time step. Note that impulse is created on the membrane due to the elastic forces, and it is diffused onto a region around the membrane. Therefore, one must place particles with zero initial impulse in a band surrounding the membrane to be the recipients of the diffused impulse. The size of the region depends on the final time of the simulation. This technique has been shown to be consistent with the heat equation and stable for a certain set of cutoff functions [18], including the one used here. A variant of this technique has also been used for boundary layer flows [5].

It is important to emphasize that in the current application the fluid fills the entire space even if the forces are restricted to the membrane. Thus the fluid velocity is the orthogonal projection of the impulse field in free space. In cases with solid boundaries, 
the projection and Laplacian operators do not commute, and so the flow near the solid boundaries require special treatment [37].

\subsection{Numerical Results for Viscous Flow}

The problem presented in this section is the same one of the previous section. The initial setup is shown in figure 7.7. The dots in the figure are Lagrangian particles that will

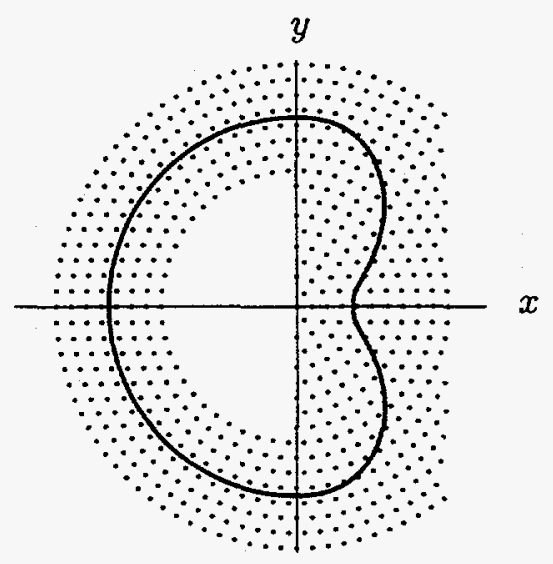

Figure 7.7: Initial membrane configuration and particle locations.

receive impulse from the diffusion process and will subsequently diffuse their own impulse; they were placed approximately 0.08 units apart. The diffusion coefficient was $\nu=10^{-3}$, the cutoff functions for the diffusion process and the impulse approximation were set equal to the one used in the previous example, but the cutoff radius for the diffusion was $\epsilon=0.1$. All other initial parameters were chosen as in the previous example.

Figures 7.8 and 7.9 show the evolution of the membrane for $0 \leq t \leq 5.52$. The motion observed in the inviscid case is now attenuated by the viscosity of the fluid and consequently, the waves propagating along the boundary are smaller. One of the important features of the viscous case is that the impulse does not remain orthogonal to the membrane due to tangential forces and the diffusion process. One implication is that the particles need not be equi-spaced as before in order to allow the tension to vary along the membrane. This eliminates the need to interpolate between particles, which in fact reduces the error in the computation of invariants. The energy in the system is not conserved due to friction but 

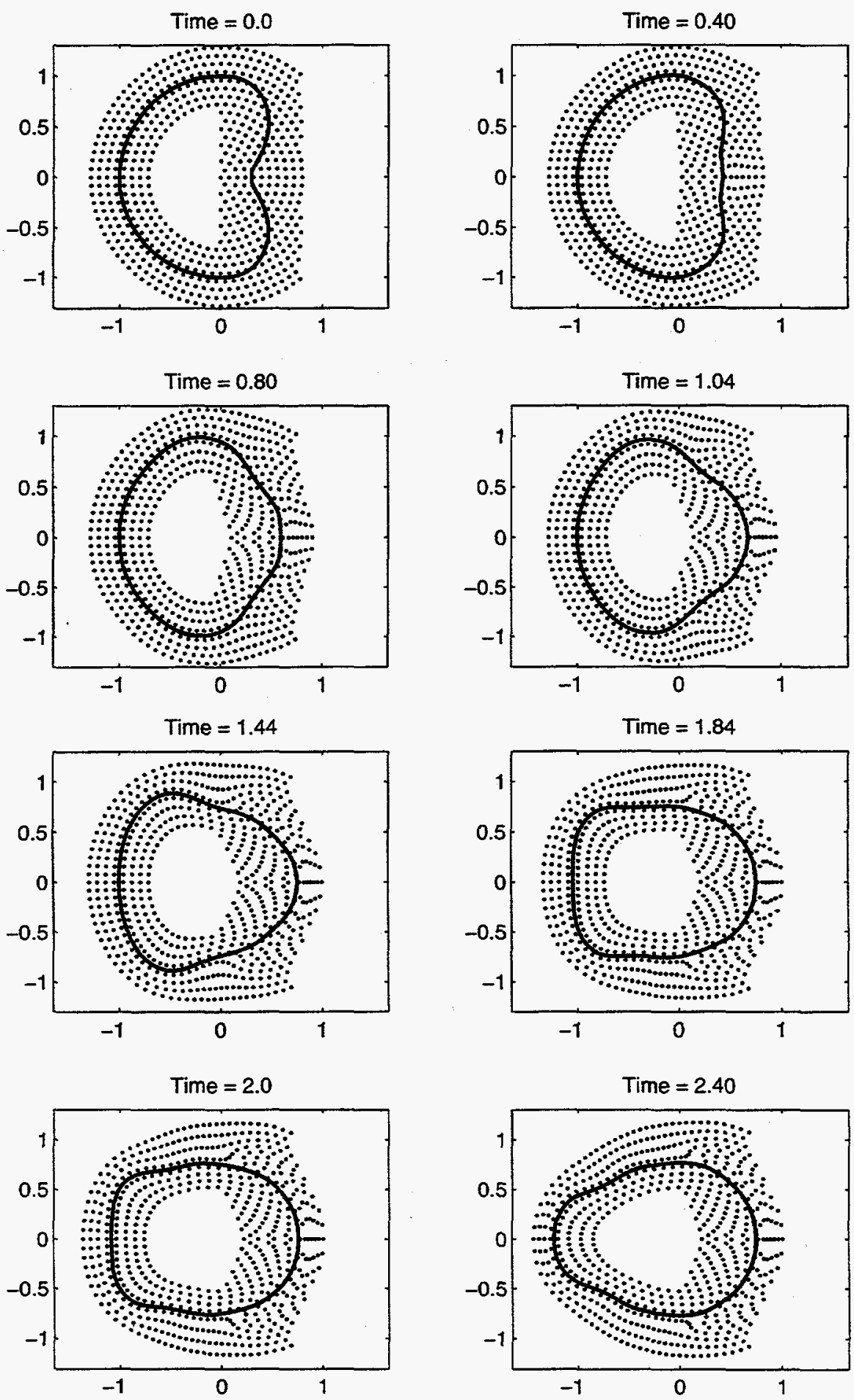

Figure 7.8: Motion of the membrane for time $t=0-2.4$, with $\Delta t=0.004$. 

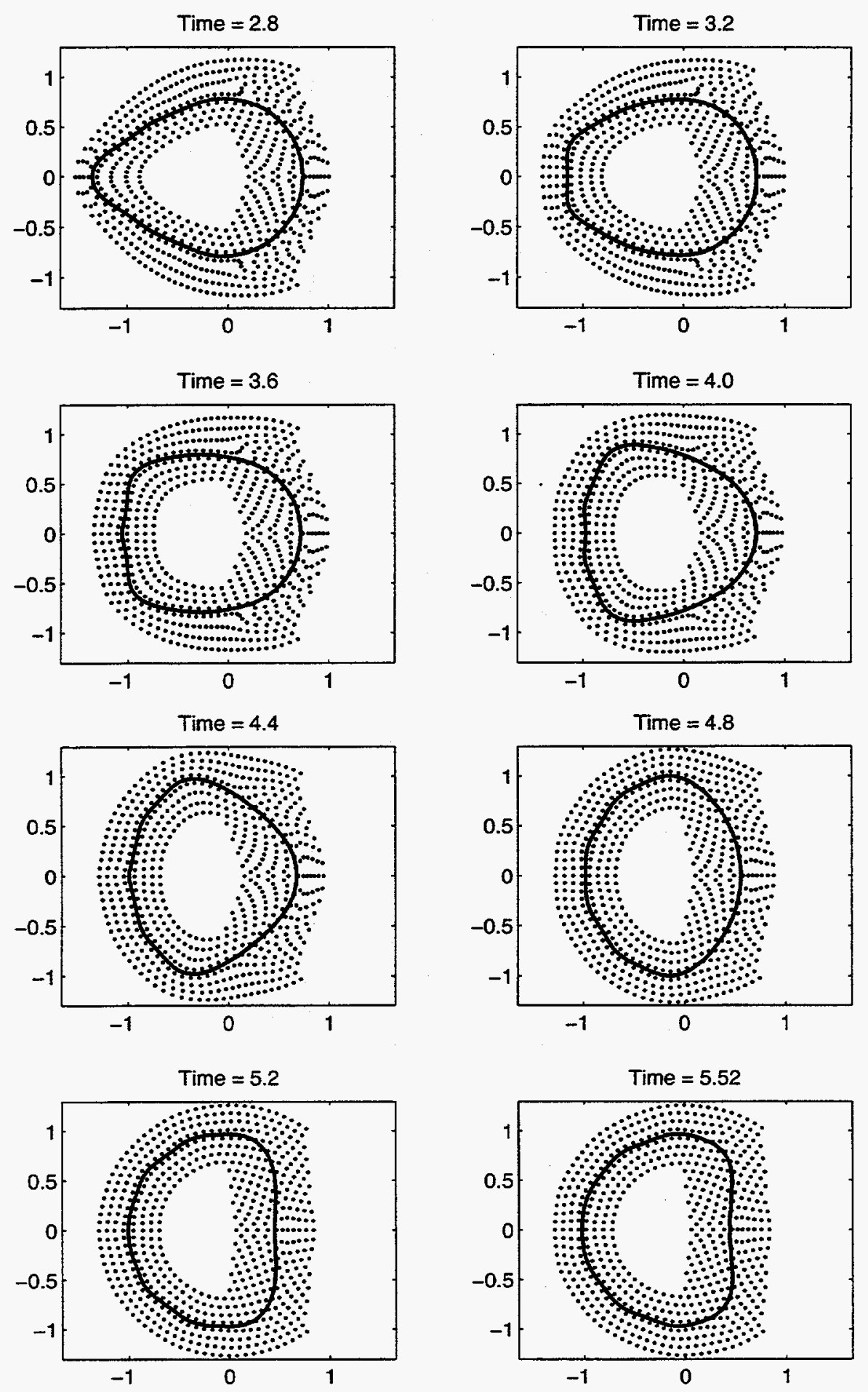

Figure 7.9: Motion of the membrane for time $t=2.8-5.52$, with $\Delta t=0.004$. 
the rate of energy loss can be computed with the formula

$$
\dot{H}(t)=\frac{1}{2} \frac{d}{d t} \int \mathbf{u} \cdot \mathbf{m} d \mathbf{x}=\nu \int \mathbf{u} \cdot \Delta \mathbf{m} d \mathbf{x} .
$$

An approximation to the integral leads to

$$
\dot{H}(t) \approx \nu \sum \mathbf{u}\left(\mathbf{x}^{j}\right) \cdot(\Delta \mathbf{m})^{j}
$$

The quantity $K+E-\dot{H}$ and the area inside the membrane are then constants of the motion. Figure 7.10 shows these invariants.
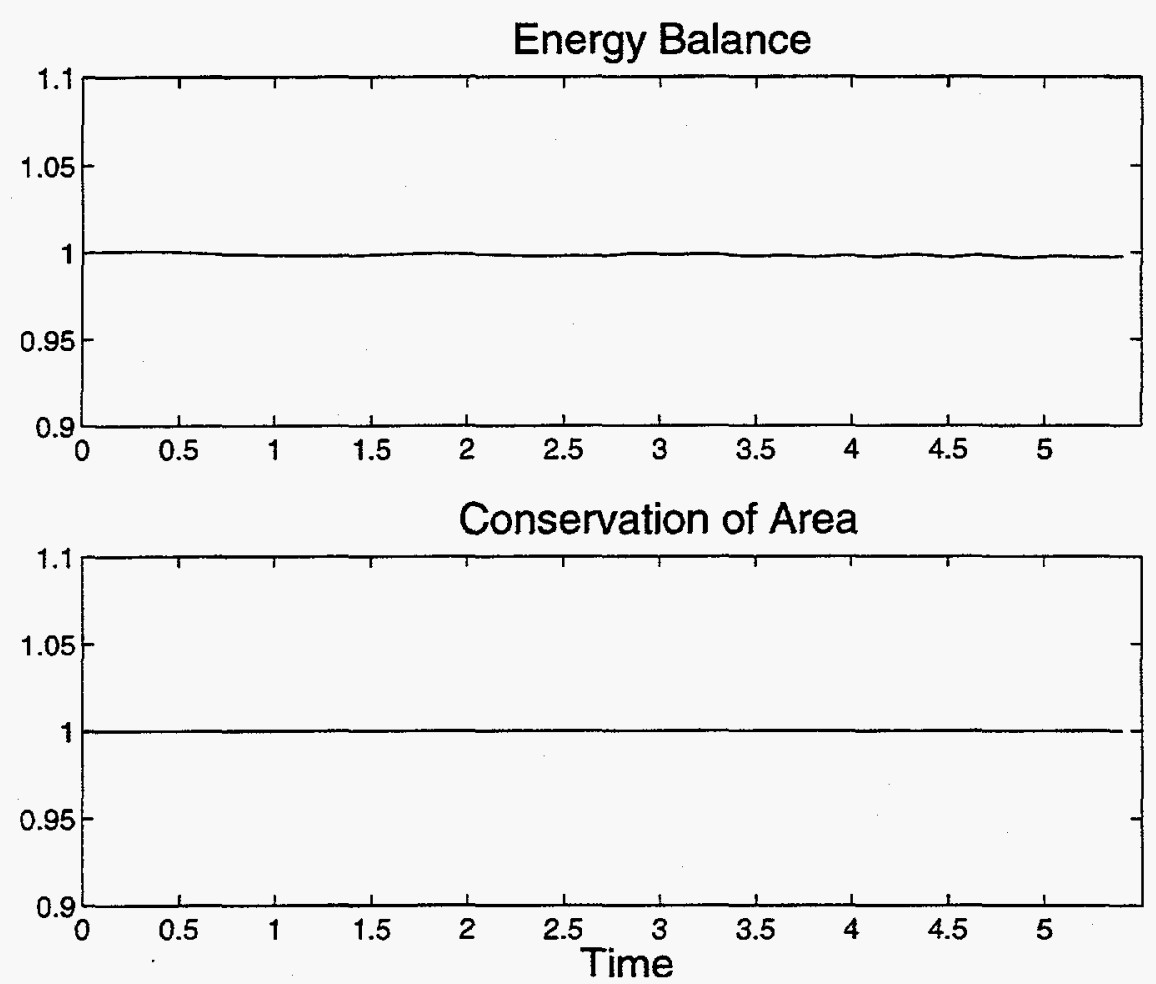

Figure 7.10: Approximate conservation of invariants for the viscous problem.

The impulse field is shown in figures 7.11 and 7.12 at times $t=2$ and $t=5$ respectively. The figures show the impulse field defined only on the membrane (top) and the impulse defined off the membrane (bottom). The bottom vectors have been amplified by a factor of 10 for clarity. The figures show that the impulse vectors on the right side of the membrane alternate in direction between inward and outward. A more detailed study of different methods for approximating the diffusion term is necessary to determine if this 
oscillation in the direction of the vectors is a manifestation of instability of the diffusion method. This study is left for future work.
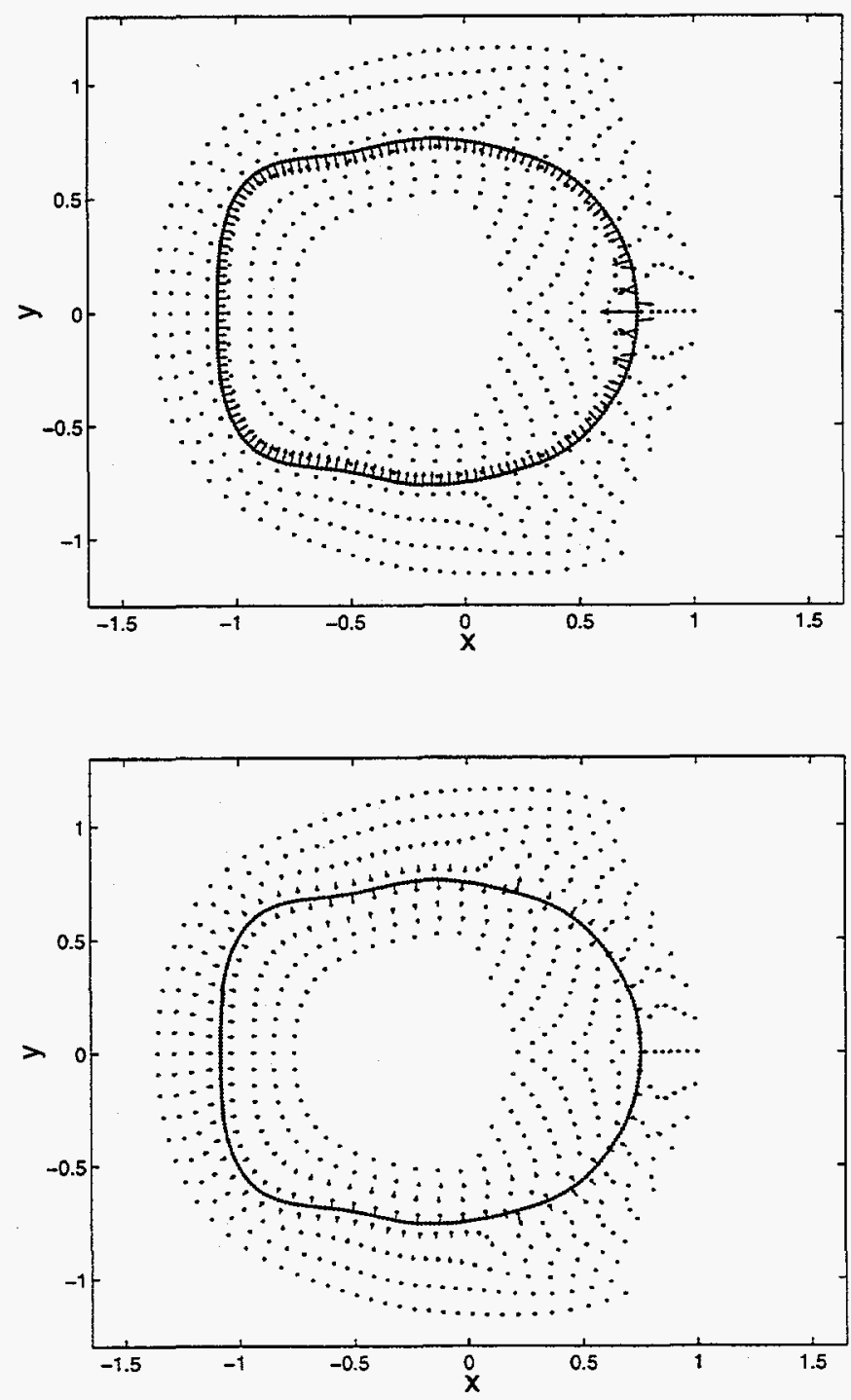

Figure 7.11: Impulse field at time $t=2$. The top picture shows the impulse on the membrane; the bottom picture shows the impulse off the membrane (magnified $\times 10$ ). 

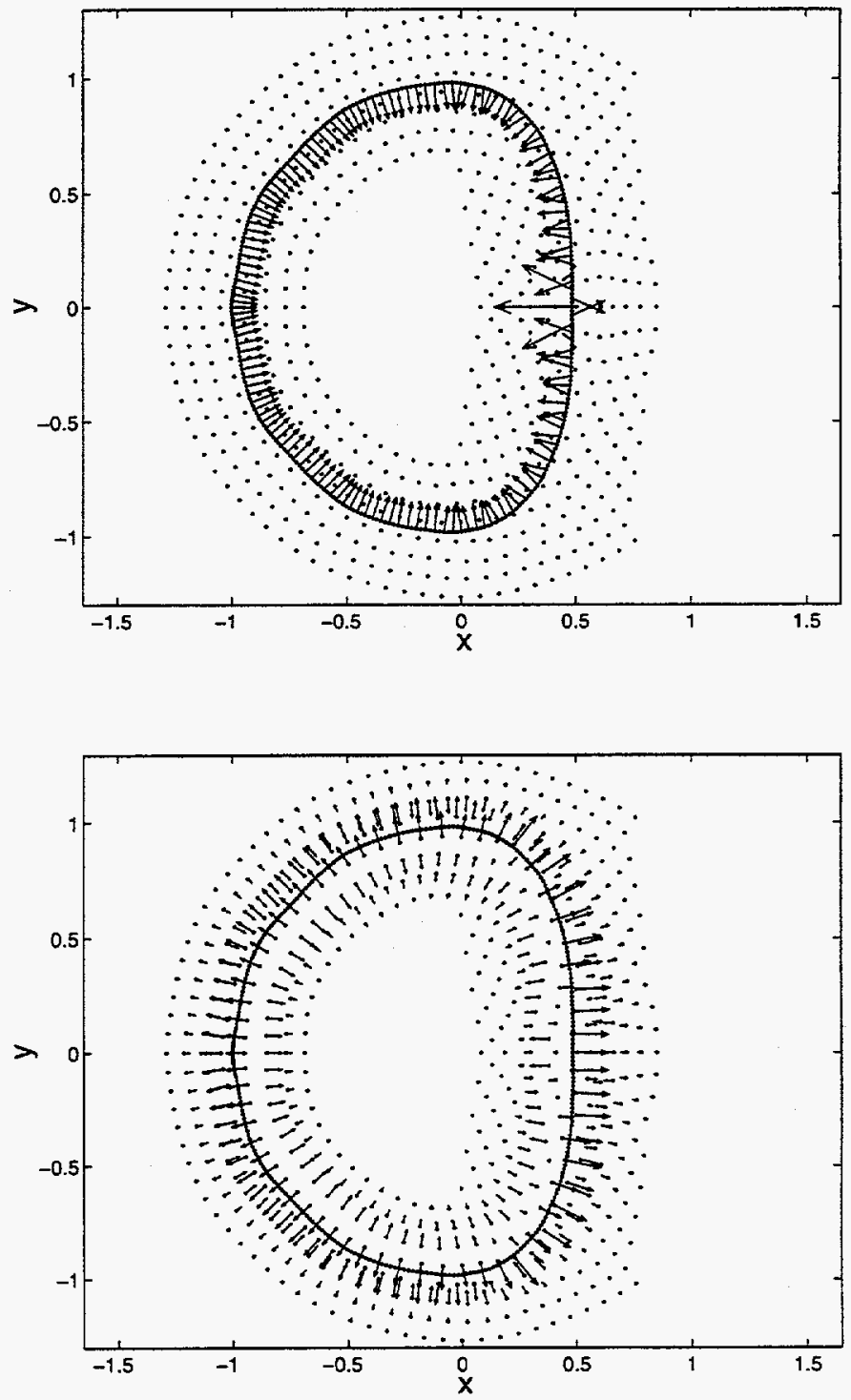

Figure 7.12: Impulse field at time $t=5$. The top picture shows the impulse on the membrane; the bottom picture shows the impulse off the membrane (magnified $\times 10$ ). 


\section{Chapter 8}

\section{Conclusions}

The connection between the velocity field due to vortex blobs and the velocity field induced by impulse blobs has been established. The connection is that the velocity field induced by two counter-rotating vortices in two dimensions can be written as a line integral along any path connecting the two vortices. An impulse field which models the same flow is an approximation of this line integral. When the integration path is carried by the flow, the path frequently stretches and the approximation of the line integral loses accuracy as the points along the path become sparse. The stretching of the path results in the growth of the magnitude of impulse vectors. In three dimensions, a large closed loop of vorticity induces a velocity field that can be approximated by an integral over any surface that spans the loop. The velocity field due to impulse is a discretization of this integral. The flow makes the surface expand, leading to the same type of accuracy loss as in $R^{2}$. This connection has been established by defining and tracking m-orthogonal surfaces, which remain normal to the impulse field in the evolution of the flow.

Based on this understanding, two techniques for avoiding the loss of accuracy in the evolution of the flow have been developed in two dimensions. One technique consists of dynamically introducing more particles along the integration path as adjacent ones drift apart. This is done in a way that exactly preserves impulse. As the discretization along the path is modified, other invariants are preserved approximately in spite of their explicit dependence on the discretization. A second technique for avoiding loss of accuracy in two dimensions involves replacing the integration path, whose endpoints are the vortices, by a shorter and more convenient one when the flow stretches the original path. The vortices are not explicitly being tracked since additional knowledge of the vorticity is not required. 
The impulse contains the information on the vorticity.

The extension of these techniques to three dimensions has been described in the context of the evolution of a thick vortex ring. A general three-dimensional resurfacing procedure requires the definition of a method for locating the vortex loops represented by impulse as well as the computation of minimal (or nearly minimal) surfaces attached to frames. While the full generality of this procedure has not been presented, the conceptual basis for it has been laid out.

One can interpret an impulse vector as a vortex dipole: the limit of two vortices of equal but opposite strength as their separation vanishes while maintaining a constant dipole moment. One consequence of this interpretation is that the high-accuracy cutoff functions of vortex methods are appropriate cutoff functions for impulse methods. A second consequence can be seen in the reconstruction of the integration path and the determination of the location of the vortices in the two-dimensional resurfacing technique. The key lies in the error analysis of the method. This analysis provides criteria for the cancellation of the vorticity created along the path except when the endpoints are encountered. Once the endpoints are found, one selects a straight line between them, defines a new impulse field along this new path, and proceeds with the computation. One illustration of this procedure is in the problem of two vortex patches, where the impulse was defined on many such integration paths in order to model the flow induced by the patches. An attractive feature of this technique is that the length of the integration paths can be kept optimal, requiring only the minimum number of particles per path for the given accuracy. With the refinement and resurfacing techniques, longer evolution times have been reached for given initial discretizations.

The interpretation of impulse variables as vortex dipoles allows us to turn to applications in which vortex dipoles are required. One such application consists of modeling the flow inside an elastic membrane immersed in a fluid. The fluid is assumed to be incompressible so that the volume of fluid inside the membrane remains constant as the membrane contracts and expands. As the elastic forces represent a rate of change of impulse, their effect on the fluid is captured by adjusting the impulse field on the boundary. The impulse generated by the forces is introduced into the fluid motion through vortex dipoles, hence the use of impulse in this problem is not only convenient but appropriate. This example is important because it is a model for problems in physiology such as blood flow in the heart chambers or air flow in the lungs. Previous methods resort to approximations of dipoles 
for the effect of the forces and lack an adequate system for updating the strengths of the dipoles. 


\section{Bibliography}

[1] C. Anderson and C. Greengard. On vortex methods. SIAM J. Numer. Anal., 22:413$440,1985$.

[2] G. K. Batchelor. An Introduction to Fluid Dynamics. Cambridge University Press, New York, 1967.

[3] J. T. Beale and A. J. Majda. High order accurate vortex methods with explicit velocity kernels. J. Comp. Phys., 58:188-288, 1985.

[4] J. B. Bell, P. Colella, and H. M. Glaz. A second order projection method for the incompressible Navier-Stokes equations. J. Comp. Phys., 85:257-283, 1989.

[5] P. S. Bernard. A deterministic vortex sheet method for boundary layer flow. J. Comp. Phys., 117:132-145, 1995.

[6] T. F. Buttke. The Hamiltonian structure of 3D fluid flow. In Proceedings of International Workshop on Vortex Flows and Related Numerical Methods, Grenoble, France, June 15-20 1992.

[7] T. F. Buttke. Lagrangian numerical methods which preserve the Hamiltonian structure of incompressible fluid flow. Comm. Pure Appl. Math., 1993.

[8] T. F. Buttke and A. J. Chorin. Turbulence calculations in magnetization variables. Appl. Num. Math., 12:47-54, 1993.

[9] Y. C. Chang, T. Y. Hou, B. Merriman, and S. Osher. A level set formulation of Eulerian interface capturing methods for incompressible fluid flow. to appear, 1994.

[10] D. L. Chopp. Computing minimal surfaces via level set curvature flow. J. Comp. Phys., 106(1):77-91, May 1993. 
[11] A. J. Chorin. Numerical solutions of the Navier-Stokes equations. Math. Comp., $22: 742-762,1968$.

[12] A. J. Chorin. On the convergence of discrete approximations to the Navier-Stokes equations. Math. Comp., 23:341-353, 1969.

[13] A. J. Chorin. Numerical study of slightly viscous flow. J. Fluid Mech., 57:785-796, 1973.

[14] A. J. Chorin. Vortex methods. Les Houches Summer School of Theoretical Physics, Les Houches, France, 1993.

[15] A. J. Chorin. Vorticity and Turbulence. Springer-Verlag, New York, 1994.

[16] A. J. Chorin and J. E. Marsden. A Mathematical Introduction to Fluid Mechanics. Springer-Verlag, New York, 1979.

[17] R. Cortez. An Approximation of Fluid Motion Due to Boundary Forces Using Impulse Variables. LBL report \#36207, September 1994.

[18] D. Fishelov. A new vortex scheme for viscous flows. J. Comp. Phys., 86:211-224, 1990.

[19] O. H. Hald. Convergence of vortex methods II. SIAM J. Sc. Stat. Comp., 16:726-755, 1979.

[20] O. H. Hald. Convergence of vortex methods for Euler's equations III. SIAM J. Num. Anal., 24:538-582, 1987.

[21] O. H. Hald. Convergence of vortex methods. In K. E. Gustafson and J. A. Sethian, editors, Vortex Methods and Vortex Motion. SIAM, 1991.

[22] J. D. Jackson. Classical Electrodynamics. John Wiley \& Sons, New York, 1975.

[23] P. Luchini. Magnetization vector analogy as a reformulation of the equations of fluid mechanics. AIAA Journal, 29:474-477, 1991.

[24] J. H. Maddocks and R. L. Pego. An unconstrained Hamiltonian formulation for incompressible fluid flow. to appear, 1994.

[25] J. E. Marsden and T. S. Ratiu. Introduction to mechanics and symmetry: a basic exposition of classical mechanical systems. Springer-Verlag, New York, 1994. 
[26] M. F. McCracken and C. S. Peskin. A vortex method for blood flow through heart valves. J. Comp. Phys, 35:183-205, 1980.

[27] R. Mendez. A vortex dipole algorithm. PhD thesis, University of California, Berkeley, 1978.

[28] H. O. Nordmark. Higher Order Vortex Methods With Rezoning. PhD thesis, University of California, Berkeley, 1988.

[29] V. I. Oseledets. On a new way of writing the Navier-Stokes equation: The Hamiltonian formalism. Commun. Moscow Math. Society, 44:210-211, 1989.

[30] C. S. Peskin. Mathematical aspects of heart physiology. Lecture notes, Courant Institute of Mathematical Sciences, 1975.

[31] C. S. Peskin. Numerical analysis of blood flow in the heart. J. Comp. Phys, 25:220-252, 1977.

[32] E. G. Puckett. A review of vortex methods. In R. Nicolaides and M. Ginzburger, editors, Incompressible computational fluid mechanics. Cambridge, 1992.

[33] P. H. Roberts. A Hamiltonian theory for weakly interacting vortices. Mathematica, 19:169-179, 1972.

[34] P. H. Rouhi. Poisson brackets for point dipole dynamics in 3D Euler flow. manuscript, 1990.

[35] P. G. Saffman. Vortex Dynamics. Cambridge University Press, 1992.

[36] J. A. Sethian. A brief overview of vortex methods. In K. E. Gustafson and J. A. Sethian, editors, Vortex Methods and Vortex Motion. SIAM, 1991.

[37] D. Summers and A. J. Chorin. A hybrid vortex/magnet method with application to flow past a sphere. in preparation.

[38] S. K. Venkatesan. Discrete singularity method and its applications to incompressible flows. $\mathrm{PhD}$ thesis, Indian Statistical Institute, Calcutta, 1993. 